\title{
BPS spectrum, wall crossing and quantum dilogarithm identity
}

\author{
DAN XIE
}

\begin{abstract}
BPS spectrum with finite number of states are found for higher rank four dimensional $\mathcal{N}=2$ theory engineered from six dimensional $A_{N-1}(2,0)$ theory on a Riemann surface with various kinds of defects. The wall crossing formula is interpreted as the quantum dilogarithm identity. Various methods including quiver representation theory, maximal green mutation, and cluster algebra are used extensively. The spectral generator and its refined version for the higher rank theory are written down using the explicit spectrum information. The finite chamber has an interesting $N^{3}$ behavior in the large $N$ limit.
\end{abstract}

1 Introduction $\quad 406$

2 Review $\quad 410$

3 Quiver representation theory 418

4 Hypermultiplets: Maximal green mutation 429

5 BPS spectrum for $A_{1}$ theory $\quad 440$

6 BPS spectrum for $A_{N-1}$ theory 460

$\begin{array}{lll}7 & \text { Spectral generator } & 499\end{array}$

8 Vector multiplets $\quad 503$

9 Conclusion $\quad 508$

$\begin{array}{lll}\text { Appendix A Explicit spectral generator } & 510\end{array}$

$\begin{array}{lll}\text { Appendix B } & \text { Refined spectral generator } & 517\end{array}$

$\begin{array}{ll}\text { References } & \mathbf{5 1 9}\end{array}$ 


\section{Introduction}

Understanding the BPS spectrum of the quantum field theory with extended supersymmetry always provides important information about the dynamics of the theory. Such objects gave the key insights to the discovery of the electric-magnetic duality of $4 \mathrm{~d} \mathcal{N}=4$ super Yang-Mills theory [1], and the exact solution of the Coulomb branch of $4 \mathrm{~d} \mathcal{N}=2$ theory [2, 3], etc.

The BPS spectrum for four dimensional $\mathcal{N}=2$ theory has the interesting wall crossing behavior, i.e. the spectrum is not smooth in crossing some marginal stability walls on the Coulomb branch. Such wall crossing behavior is very important for the consistency of the solution $[2,3]$. The SeibergWitten solution gives us the mass formula for the BPS particle, but it does not teach us explicitly the BPS spectrum at a given point on the Coulomb branch. Therefore, the BPS spectrum is only found for very few examples in the early days, say $S U(2)$ with $N_{f} \leq 4$ and $S U(2)$ with massive adjoint [4-7].

Quite recently, Mathematician proposed a remarkable wall crossing formula which constructed an invariant quantity from the BPS spectrum [8-10]. Physical understandings of the wall crossing formula using the Hyperkahler metric of the Coulomb branch of the corresponding three dimensional theory are given in [11]. However, the formula itself does not give us the answer of the BPS spectrum of a given $\mathcal{N}=2$ theory, and finding the BPS spectrum is still a very difficult problem even with lots of exciting development in the past few years [12-23], in particular, very few information is known for the higher rank theory. The main purpose of this paper is to find the explicit BPS spectrum for a large class of higher rank theories.

We are going to focus on a particular class of theory called theory of class $\mathcal{S}$ which can be engineered by compactifying six dimensional $(2,0)$ theory $A_{N-1}$ theory on a Riemann surface with regular and irregular singularities $[13,24,25]$. This class is very huge, for example, it includes generalized superconformal quiver gauge theory[24, 26], general Argyres-Douglas type theories [25, 27, 28], and lots of new asymptotical free theories [25]. Most of theories in this class are strongly coupled, but the remarkable geometrical construction can tell us many properties of them including superconformal index [29], 3d mirror [30], etc, and this class is a golden arena for studying the dynamics of the quantum field theory.

In this paper, we would like to study the BPS spectrum of all kinds of theories from class $\mathcal{S}$. Previous studies of BPS spectrum and wall crossing mainly focused on the $A_{1}$ theory, and the geometrical approach using the flow lines on the Riemann surface [13] and the equivalent quiver approach 
$[20,21,31]$ have the tremendous success in understanding the BPS spectrum of these theories, however very little is known for the higher rank cases ${ }^{1}$. It seems that these higher rank theories are much more difficult than the $A_{1}$ theory since the underlying combinatorics is much harder.

Our results presented in this paper show that the finite chamber of the higher rank theory in this class can be easily worked out by combining the geometric construction and the quiver approach: we can find explicitly the charges and the order of phases of these particles. To achieve this goal, we have the following two main assumptions:

1. The triangulation and the network from the corresponding Riemann surface constructed in [33-35] give the BPS quiver of the theory, and the potential of the quiver is also given, see Figure 1.

2. The Donadson-Thomas invariant from the quiver with potential encodes the BPS spectrum, and the factorization depends on the $\theta$ stability condition of the quiver representation theory [36].
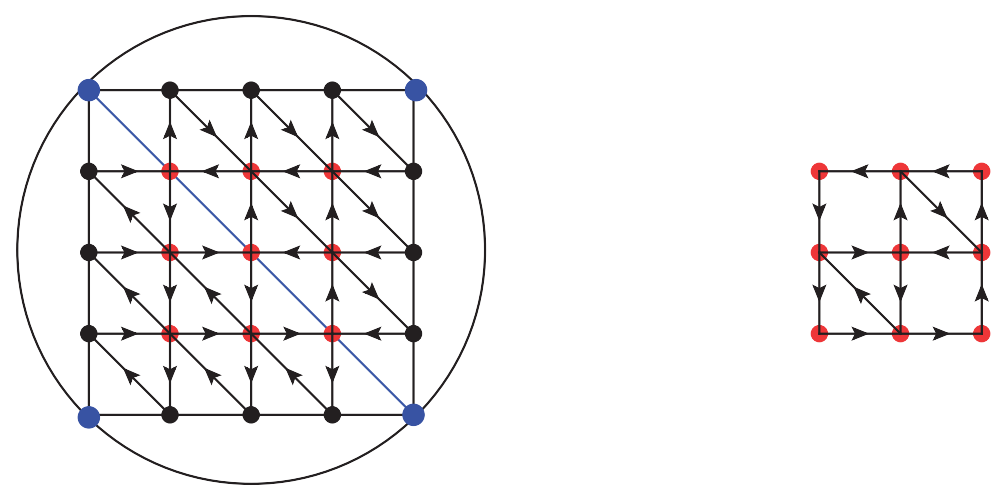

Figure 1: The triangulation of fourth punctured disc of $A_{3}$ theory which represents the $\left(A_{3}, A_{3}\right)$ Argyres-Douglas theory. The quiver is shown on the right.

The Donadson-Thomas invariant is in fact very complicated for the general quivers, and it is hard to extract the spectrum even the invariant is found. However, for the finite chamber, the BPS spectrum can be found using a remarkable combinatorial method called maximal green mutation

\footnotetext{
${ }^{1}$ One chamber for $T_{3}$ theory and one single gauge group with various fundamentals are worked out in [21], and some weakly coupled chambers of pure $S U(N)$ theory are studied in [32]; Two chambers for the sphere with one type of irregular singularity is worked out in [16], and this class is also considered in [23].
} 
introduced by Keller [37] (which is equivalent to the mutation method proposed in [21] and is used implicitly in [13]). Basically, green mutation is defined by first extending the quivers by adding an extra frozen quiver node to each node, see Figure 2a. Now each node is defined as green (red) if it is the source ( sink) of the frozen node. The green mutation sequence is defined as mutating only the green nodes, and the maximal green mutation is defined as the green mutation sequences such that no green node is left, see Figure $2 \mathrm{~b}$. The road map for finding the finite chamber is

Step 1. Extend the BPS quiver by adding a frozen node to each original quiver node.

Step 2. Define a charge vector $\gamma_{i}$ for the frozen nodes satisfying $\left\langle\gamma_{i}, \gamma_{j}\right\rangle=$ $\epsilon_{i j}$ with $\epsilon_{i j}$ the antisymmetric matrix for the quiver, then each green mutation on a node $k$ probes a BPS hypermultiplet with charge $\alpha=\sum_{i} n_{i} \gamma_{i}$ where $n_{i}$ is the number of arrows from node $k$ to the ith frozen node.

Step 3. Find the maximal green mutation sequences such that no green node is left.

The green mutation sequences give the phase order and the charge vector automatically. In practice, the Java program in [38] developed by Keller is extremely useful for us to find the maximal green mutation sequences for the complicated quiver.

Now for each maximal green mutation sequences $\mathbf{k}=\left(k_{1}, \ldots, k_{s}\right)$, one can associate a quantum dilogarithm product [37]

$$
E(\mathbf{k})=E\left(X^{\alpha_{1}}\right) \cdots E\left(X^{\alpha_{s}}\right)
$$

here $E(x)$ is the familiar quantum dilogarithm function and $X^{\alpha}$ is operator satisfying the noncommutative relation

$$
X^{\alpha} X^{\beta}=q^{1 / 2\langle\alpha, \beta\rangle} X^{\alpha+\beta},
$$

with $\langle\alpha, \beta\rangle$ the familiar Dirac product of two charges. If there is another sequences $\mathbf{k}^{\prime}=\left(k_{1}^{\prime}, \ldots, k_{r}^{\prime}\right)$ which represents the spectrum in another chamber, then the wall crossing formula is the following quantum dilogarithm identity $E(\mathbf{k})=E\left(\mathbf{k}^{\prime}\right)$. From two chambers shown in Figure 2, we have

$$
E\left(X_{1}\right) E\left(X_{2}\right)=E\left(X_{2}\right) E\left(q^{-\frac{1}{2}} X_{1} X_{2}\right) E\left(X_{1}\right)
$$

which is the basic quantum dilogarithm identity found in [39]. 


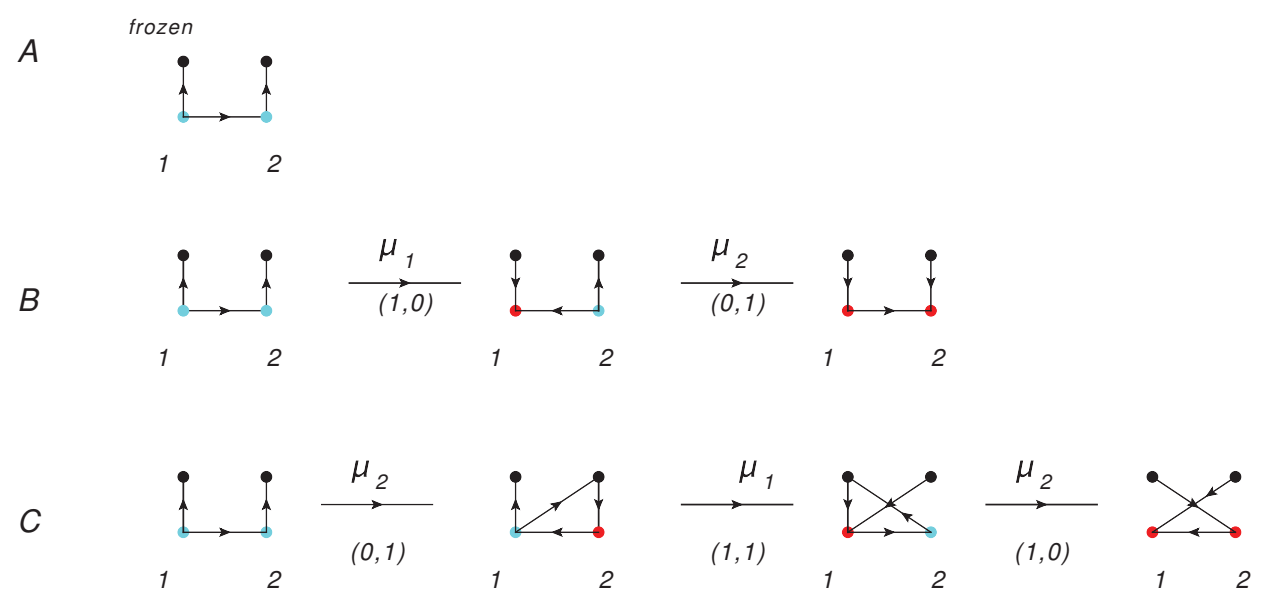

Figure 2: A: The extended quiver derived by adding extra frozen node. B: A maximal green mutation sequence and the charge is indicated for each green mutation. C: A different maximal green mutation sequence.

The above combinatorial method is very useful and in principle one could try all possible green mutations, but in practice it has very limited uses if one does not know the mutation structure of the quiver. The geometric picture of the triangulation of the bordered Riemann surface from which the quiver is derived turns out to be very useful in finding maximal green mutation sequence. We are going to give many concrete examples showing explicitly the mutation sequences. In some cases, the result is very elegant, for example, we find that the minimal chamber of $T_{N}$ theory has the following number of states

$$
N_{\text {min }}=2 N(N-1)^{2},
$$

when $N=5$, the minimal chamber has 160 states, and we can find the charge vectors and the phase order explicitly which is very hard to derive without knowing some structures of the quivers!

Another use of our result is that we can write down the spectral generator [13] pretty easily for the higher rank theory since the final cluster coordinates (identified with the spectral generator) associated with the quiver nodes can be derived given the mutation sequence. A remarkable feature of maximal green mutation sequence is that it tracks the permutation of the quiver nodes, so we can identify the final cluster coordinates of the original quiver node, which do not depend on the specific chamber. 
This paper is organized as follows: Section 2 reviews some backgrounds about the BPS particles, quivers, and cluster algebra; Section 3 discusses how to use the quiver representation theory and the $\theta$ stability condition to find the BPS spectrum; Section 4 discusses using the combinatorial tools called maximal green mutation to find the finite chamber; Section 5 and section 6 discuss the finite chamber of the $A_{1}$ theory and $A_{N-1}$ theory with many examples; Section 7 shows how to write the spectrum generator for the higher rank theory; Section 8 discusses briefly the chamber with vector multiplets; We give a short conclusion in section 9 .

\section{Review}

\subsection{Generality about wall crossing}

The exact solution of Coulomb branch of four dimensional $\mathcal{N}=2$ theory is solved by Seiberg and Witten in [2,3]. Let's take pure $S U(2)$ theory as an example in which the gauge group is broken to $U(1)$ at a generic point of Coulomb branch, and there are singularities on the Coulomb branch where extra monopoles or dyons become massless.

The $\mathcal{N}=2$ supersymmetry algebra allows a central charge extension, and the central charge of a BPS particle with charge vector $\gamma=\left(n_{e}, n_{m}\right)$ is described by

$$
Z_{\gamma}(u)=n_{e} a(u)+n_{m} a_{D}(u)
$$

where $a$ is the scalar component in the vector multiplet and $a_{D}$ is the dual variable, and the central charge depends on the coordinate $u$ of the Coulomb branch. The BPS particle with charge $\gamma$ has mass $M(\gamma)=|Z(\gamma)|$. As discussed in Seiberg and Witten's original paper, the wall crossing behavior of these BPS particles is important for the consistency of the solution. Basically, a BPS particle with charge $\gamma$ can decay to other BPS particles, say $\gamma_{1}$ and $\gamma_{2}$, in crossing the marginal stability wall. Due to the BPS condition and charge conservation, this is only possible if their central charges have the same phases:

$$
\begin{aligned}
& \gamma=\gamma_{1}+\gamma_{2}, \\
& \arg Z(\gamma)=\arg Z\left(\gamma_{1}\right)=\arg Z\left(\gamma_{2}\right)
\end{aligned}
$$

For pure $S U(2)$ theory, there is only one marginal stability wall for all the particles, see Figure 3. There are only two chambers: one has finite number 
of hypermultiplets and the other chamber has a $W$ boson and an infinite number of dyons. All of the particles but two from chamber 1 become unstable in crossing the wall. The above wall crossing behavior for pure $S U(2)$

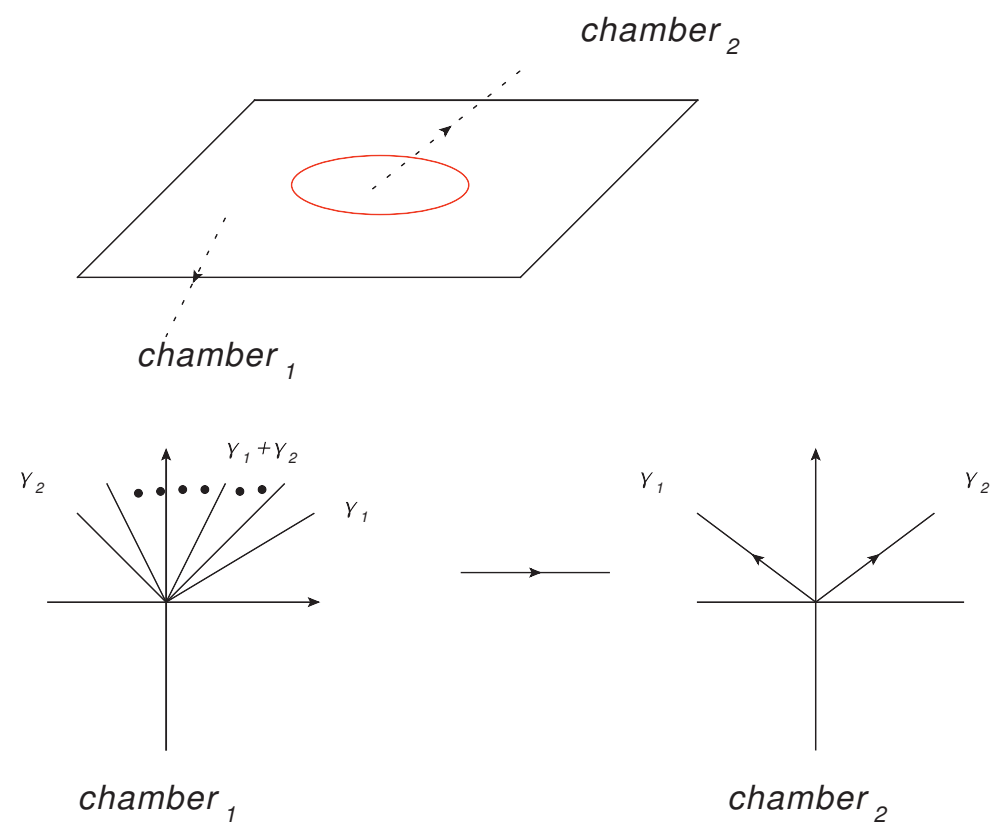

Figure 3: Top: The marginal stability wall of pure $S U(2)$ theory. Bottom: The BPS spectrum in two chambers, and there are infinite number of states in one chamber and finite states in another chamber.

theory happens in the strongly coupled region. However, wall crossing behavior is not tied to the strongly coupled effect, and it can also happen in the weakly coupled region as discussed in [3].

The BPS particle for a general $\mathcal{N}=2$ theory has charge vector $\gamma=$ $\left(n_{e}^{i}, n_{m}^{i}, s^{f}\right)$, here $n_{e}^{i}$ and the $n_{m}^{i}$ are the electric and magnetic charge of the $i$ th $U(1)$ gauge group, and $s^{f}$ is the flavor charge. Therefore the rank of the charge lattice is $R=2 n_{r}+n_{f}$, where $n_{r}$ is the rank of the gauge group and $n_{f}$ is the number of mass parameters. Two charge vectors have a natural antisymmetric Dirac product

$$
\left\langle\gamma_{1}, \gamma_{2}\right\rangle=n_{e}^{i} p_{m}^{i}-n_{m}^{i} p_{e}^{i}
$$

Note that the product does not depend on the flavor charge, so the rank of a matrix formed by an independent basis of BPS particles is $2 n_{r}$. The central 
charge for a BPS particle with charge $\gamma$ is

$$
Z(\gamma)(u)=n_{e}^{i} a^{i}(u)+n_{m}^{i} a_{D}^{i}(u)+s_{f} m_{f} .
$$

This formula does not tell us which charge vectors are allowed as a possible BPS particles, and it also does not tell us which BPS particle is stable, so one need extra analysis to find the BPS spectrum. By finding the BPS spectrum of a given $\mathcal{N}=2$ theory, we mean to find the charge vectors and their order of phases of all the stable BPS particles. All BPS states in $3+1$ dimensions have at least a half-hypermultiplet (i.e. a hypermultiplet without its CPT conjugate) of spin degrees of freedom. The CPT conjugate has opposite phase and same masses, so we will only consider half of the BPS particles in all later study.

To count the number of BPS states, one can define a helicity supertrace for a charge vector $\gamma$ ( see [40] for details):

$$
\Omega(\gamma, u)=-\frac{1}{2} \operatorname{Tr}_{\mathcal{H}_{\gamma}}(-1)^{2 J_{3}}\left(2 J_{3}\right)^{2}=(-1)^{2 j}(2 j+1) .
$$

This index receives contributions from BPS particles (short representation of the SUSY algebra), and $\Omega(\gamma)=1$ for the hypermultiplet, and $\Omega(\gamma)=-2$ for the vector mulitplet. To track the spin content, a refined index can be defined [18]:

$$
\Omega(\gamma, y, u)=\operatorname{Tr}_{\mathcal{H}_{\gamma}}(-1)^{2 J_{3}} y^{2 J_{3}+2 I_{3}} .
$$

The hypermultiplet contributes $\Omega(\gamma, y)=1$ and the value for the vector multiplet is $\Omega(\gamma, y)=y+y^{-1}$. Since the BPS spectrum is only piece-wise constant across the Coulomb branch, the above index is not an invariant. Konsetvich and Soilbeman (KS) came up with a remarkable wall crossing formula which basically constructed a Donaldson-Thomas (DT) invariant from the BPS spectrum. They first associate a quantum torus algebra $e_{\gamma}$ on the charge lattice

$$
\left\langle e_{\gamma_{1}}, e_{\gamma_{2}}\right\rangle=(-1)^{\left\langle\gamma_{1}, \gamma_{2}\right\rangle}\left\langle\gamma_{1}, \gamma_{2}\right\rangle e_{\gamma_{1}+\gamma_{2}}
$$

and then define a group element for a BPS particle with charge $\gamma$ :

$$
U_{\gamma}=\exp \left(\frac{1}{n^{2}} e_{n \gamma}\right)
$$


and the invariant from the BPS spectrum are given by an ordered product:

$$
A=\sum_{\gamma} U_{\gamma}^{\Omega(\gamma, u)}
$$

The ordered product is taken over the particles with decreasing phases. The KS wall crossing formula states that this product is independent of the BPS chamber!

The KS wall crossing formula is very beautiful. However, it is still not enough to find the BPS spectrum for a given $\mathcal{N}=2$ quantum field theory. To apply the wall crossing formula, one need to know at least the spectrum of one chamber and then apply the known wall crossing formula. In practice, usually nothing is known for the BPS spectrum of a given theory, and even if we know the spectrum of one chamber, it seems hard to find other chambers using the wall crossing formula.

The quiver approach, on the other hand, provides hope of solving the BPS spectrum in practice. The idea is to attach a unique quiver to a given $\mathcal{N}=2$ theory, and then use various tools attached to the quiver to study the BPS spectrum, since there are many wonderful properties about the quivers, the BPS counting problem is actually much easier. The factorization of the DT invariant for a quiver with potential is an important class studied by Kontesvich and Soilbman [8]. and the quiver approach to study the BPS spectrum has been used successfully in the early study of the wall crossing in the physics literature [41-43]. In this paper, we will start with a quiver with potential for a large class of $\mathcal{N}=2$ theory, and since the BPS information is encoded in this quiver, it is called BPS quiver.

\subsection{BPS quiver for $\mathcal{N}=2$ theory}

Let's review some background on BPS quiver which could be understood directly from the spectrum at a given point on the Coulomb branch [21]. Given a UV complete $\mathcal{N}=2$ field theory, let's assume that we know explicitly the central charges and the stable BPS spectrum in one chamber, and each BPS particle can be represented by a ray in the complex plan. Since there is always an antiparticle whose phase is opposite to it, we only need to focus on a half plane. However, such choice is arbitrary which leads to many equivalent descriptions.

Let's take a half-plane $S_{0}$ and all the BPS particles fall into this region. A canonical basis for these BPS states is defined as follows: the basis is chosen such that the charge of any BPS state in this half plane is expressed 
as a sum of this basis with positive integer coefficient

$$
\gamma=\sum n_{i} \gamma_{i}
$$

where $n_{i}$ is a non-negative integer. It is easy to show that such basis is unique (again using the positivity property). Now a BPS quiver can be formed by taking the Dirac product of this basis and forming an antisymmetric matrix

$$
\epsilon_{i j}=\left\langle\gamma_{i}, \gamma_{j}\right\rangle
$$

which defines a quiver. From this definition, it is easy to see that the left and right-most of states should be included into this basis, since they can not be written as the sum of other charges with the positive coefficient. This basis is not always possible, and usually one need to turn on all the mass deformations of the theory and it turns out that a BPS quiver can be found for a large class of theories considered in this paper, which will be discussed in more detail later.

The basis would be different if we choose a different half-plane $S_{1}$. If we slightly rotate the region $S_{0}$ clockwise and choose $S_{1}$ such that only the left-most particle $\gamma_{L}$ drops out, then the charge vector $-\gamma_{L}$ which is in the right-most of $S_{1}$ has to be included in the new basis, see Figure 4, and the charge $\gamma_{L}$ is dropped out. Moreover, other charge vectors should also be changed, and the new basis is assumed to take the following form (see a proof in $[21])$ :

$$
\begin{aligned}
& \gamma_{L}^{\prime}=-\gamma_{L}, \\
& \gamma_{i}^{\prime}=\gamma_{i}+\left[\epsilon_{i L}\right]_{+} \gamma_{L},
\end{aligned}
$$

here $\left[\epsilon_{i L}\right]_{+}=\max \left[0, \epsilon_{i L}\right]$. Now the new antisymmetric tensor built from the canonical basis is different and we have a new quiver, which is equally good to capture the BPS spectrum. So the BPS quiver is not just a single quiver but a family of quivers related by the above transformation on basis called quiver mutations (we will explain this term later), and by BPS quiver we really mean its mutation class.

The above definition of the BPS quiver is conceptually good but not useful for finding the BPS quiver since usually the BPS spectrum is not known for any chamber. Other approaches are needed to attach a quiver to a given $\mathcal{N}=2$ theory. For a large class of $\mathcal{N}=2$ theory engineered from compactifying six dimensional higher rank ${ }^{2} A_{N-1}$ theory on a Riemann

\footnotetext{
${ }^{2}$ The BPS quiver for $A_{1}$ theory is discussed in [13, 31].
} 

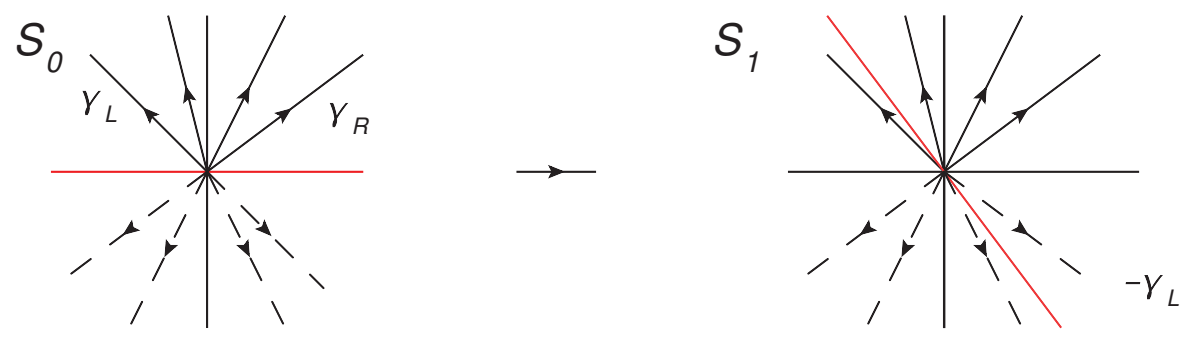

Figure 4: By rotating the half plane, the canonical basis is changed.

surface $\Sigma$ with defects, one could associate a quiver from the combinatorial data of the Riemann surface. Our main conjecture in this paper is that

Conjecture 1. BPS quiver for these theories is the one found from the triangulation of a bordered Riemann surface as described in [33-35].

There are many compelling evidence that this conjecture is true, i.e. the rank of the quiver is always equal to twice of the Coulomb branch dimensions, and the results are in agreement with the quiver found using other approaches like $2 \mathrm{~d}-4 \mathrm{~d}$ correspondence.

\subsection{Quiver mutation and cluster algebra}

After finding a quiver, there are many tools one could use to study the BPS spectrum like the quiver representation theory, stability and quiver moduli space [42], etc, which we will review in detail in next section. One could also attach new combinatorial structure called cluster algebra [44] on the quiver which proves to be very powerful in BPS counting. There is a huge amount of literature on cluster algebra, here we only review some basic definitions which is sufficient for our purpose. The first element of the cluster algebra is the quiver mutations, which is a combinatorial operation acting on quiver in following way:

$$
\epsilon_{i j}^{\prime}= \begin{cases}-\epsilon_{i j} & \text { if } i=k \text { or } j=k \\ \epsilon_{i j}+\operatorname{sgn}\left(\epsilon_{i k}\right)\left[\epsilon_{i k} \epsilon_{k j}\right]_{+} & \text {otherwise }\end{cases}
$$

Notice that this definition is the same as the change of the canonical basis shown in last subsection. The quiver mutations can be represented beautifully using the quiver diagram: A quiver is a directed graph where multiple 
arrows between two vertices are allowed, which is derived using $\epsilon_{i j}$ as follows: attach a quiver node for $i=1, \ldots, n$, and there are $\epsilon_{i j}$ arrows between node $i$ and node $j^{3}$. The quiver mutation for a quiver without one and two cycles (such quiver is called 2-acyclic) is defined as the following: Let $\mathrm{Q}$ be a quiver and $\mathrm{k}$ a vertex of $\mathrm{Q}$. The mutation $\mu_{k}(Q)$ is the quiver obtained from $\mathrm{Q}$ as follows, see Figure 5:

1) for each sub quiver $i \rightarrow k \rightarrow j$, create a new arrow between $i j$ starting from $i$;

2) we reverse all arrows with source or target $k$;

3 ) we remove the arrows in a maximal set of pairwise disjoint 2-cycles.
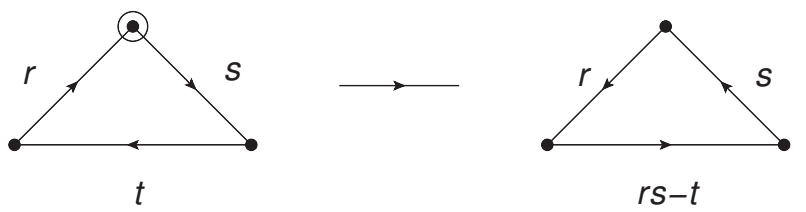

Figure 5: The quiver mutation.

Formally, the quiver mutation is exactly like the Seiberg duality [45] for four dimensional $\mathcal{N}=1$ quiver gauge theory: the quarks are transformed to antiquarks and vice versa, and there is a new singlet for each meson field in the original quiver; Finally the potential term is used to integrate out the massive fields. There are several obvious features about the quiver mutations: a. $\mu_{k}$ is invertible and $\mu_{k}^{2}=1$. b. If there are no quiver arrows between two quiver nodes $i$ and $j$, then $\mu_{i}$ and $\mu_{j}$ commute.

2.3.1. Quiver with potential. If there are oriented cycles in the quiver, one can define a potential term $W$ as familiar from the quiver gauge theory (a gauge invariant operator). The quiver mutation acting on the quiver itself is the same form as the Seiberg duality, similarly, the mutation action on the potential [46] is also the same as what is happening in the context of Seiberg duality. If there is an oriented path $\cdots i \rightarrow^{\alpha} k \rightarrow^{\beta} j \cdots$ passing through the node $k$ under mutation, and the potential involving this piece has the form

$$
W=\cdots \alpha \beta \cdots+\cdots ;
$$

In doing the Seiberg duality, the bi-fundamental fields $\alpha$ and $\beta$ change the orientations, which are denoted as $\alpha^{*}$ and $\beta^{*}$ in the new quiver, and there is

\footnotetext{
${ }^{3}$ If $\epsilon_{i j}$ is positive, the quiver arrows are pointing from node $i$ to node $j$; otherwise, they are pointing from node $j$ to node $i$.
} 
a new singlet bifundamental field $[\alpha \beta]$ between node $i$ and $j$. The potential changes in the following way: the $\alpha \beta$ term in the original potential is replaced by the new field $[\alpha \beta]$, and there is an extra cubic potential term for the new quarks and the singlet:

$$
W^{\prime}=\cdots[\alpha \beta] \cdots+\beta^{*} \alpha^{*}[\alpha \beta]+\cdots
$$

Now there might be a quadratic term in $W^{\prime}$ which means that there are two cycles in the new quiver, and the existence of the potential can be used to integrate out these fields, and we get a reduced quiver $Q_{\text {reduced }}$ and a reduced potential $W_{\text {reduced }}$, see Figure 6 . The quiver and potential after the mutation are always the reduced one!

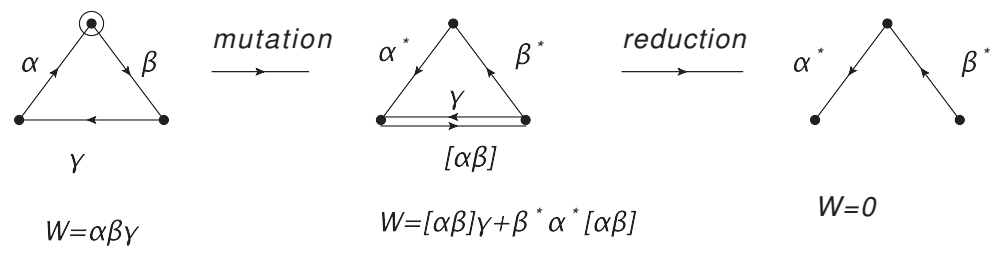

Figure 6: The quiver mutation for the quiver with potential, and the final quiver is the reduced one.

2.3.2. Cluster $X$ variable and cluster $\boldsymbol{A}$ variable. A complex variable $X_{i}$ can be defined on each quiver nod ${ }^{4}$, and its transformation behavior under the quiver mutation acting on node $k$ is

$$
X_{j}^{\prime}= \begin{cases}X_{k}^{-1} & \text { if } j=k \\ X_{j}\left(1+X_{k}^{-\operatorname{sgn}\left(\epsilon_{j k}\right)}\right)^{-\epsilon_{j k}} & \text { if } j \neq k\end{cases}
$$

namely, only the $X$ variable of the quiver nodes connected to node $k$ is changed.

\footnotetext{
${ }^{4}$ In cluster algebra literature, this $X$ variable is called coefficient and denoted as $y$, and the cluster $A$ variable is denoted as $x$, here we follow the convention of Fock-Goncharov [33]
} 
Similarly, another set of $A$ variable isdefined on each quiver node, and the transformation behavior of the $A_{k}$ under the quiver mutations are

$$
A_{k}^{\prime}=\frac{\prod A_{i}^{\left[\epsilon_{i k}\right]_{+}}+\prod A_{i}^{\left[-\epsilon_{i k}\right]_{+}}}{A_{k}}
$$

where $\left[\epsilon_{i k}\right]_{+}=\max \left[\epsilon_{i k}, 0\right]$, and other $A$ variables are not changed. There is an interesting duality between the $X$ and $A$ variables

$$
X_{i}=\sum_{j} A_{j}^{\epsilon_{i j}} .
$$

Therefore cluster algebra is formed by a lot of seeds, and each seed comprises of quadruple $\left(\epsilon_{i j}, W, X_{i} A_{i}\right)$, and the seeds are related by the quiver mutation formula $(17,19,20,21)$. A degenerate two form can be defined on $A$ space

$$
\omega=\epsilon_{i j} d \log A_{i} \wedge d \log A_{j} ;
$$

and a poisson structure can be defined on the $X$ space:

$$
\left\{X_{i}, X_{j}\right\}=\epsilon_{i j} X_{i} X_{j}
$$

These structures are compatible with the cluster transformation, i.e. if you express $\left(X_{i}, \epsilon_{i j}\right)$ in terms of $\left(X_{i}^{\prime}, \epsilon_{i j}^{\prime}\right)$ using the cluster transformation rule, and you will get the same form expressed in terms of $\left(X_{i}^{\prime}, \epsilon_{i j}^{\prime}\right)$.

\section{Quiver representation theory}

\subsection{Acyclic quiver and BPS spectrum}

We review the idea of using quiver representation theory and $\theta$ stability condition to find the BPS spectrum, and this subsection is mainly following [36]. The quiver considered in this section is assumed to be acyclic, i.e. there is no closed oriented path in the quiver.

Let's first discuss some backgrounds on the quiver representation theory and its moduli space. The content reviewed below is quite standard and more details could be found in many mathematical literature, i.e. the review by Reneke [47]. Let's denote the quiver as $Q$, and $Q_{0}$ as the set of quiver nodes, $Q_{1}$ as the set of quiver arrows. A representation $V$ of $Q$ consists of complex vector spaces $V_{i}$ for $i \in Q_{0}$ of dimension $d_{i}$, and of linear maps $V_{\alpha}: V_{i} \rightarrow V_{j}$ for every arrow $\alpha: i \rightarrow j$ in $Q$. Physically, a quiver representation can be 
thought of as assigning a $U\left(d_{i}\right)$ gauge group on each vertex and the linear map is the expectation value of the scalar in the bifundamental matter. The homomorphism $\phi$ between two quiver representations are a set of linear maps

$$
\phi_{i}: V_{i} \rightarrow V_{i}^{\prime}
$$

which preserves the structure of the quiver representation, i.e $V_{\alpha}=\phi_{i} V_{\alpha}^{\prime} \phi_{j}^{-1}$. Again, when the dimension vectors of two representations are same, this is the familiar gauge transformation acting on the bi-fundamental fields. The endomorphism of a quiver representation is the morphism between itself, and the set of all endomorphism of a representation is denoted as $\operatorname{End}(V)$. The automorphism is the the endomorphism which is also invertible, and the set of all the automorphism of a representation is denoted as $A u t(V)$.

A representation $N$ is a subrepresentation of $M$ if $N_{i} \subset M_{i}$ for all the quiver nodes and the map $M_{\alpha}$ satisfies the condition $M_{\alpha}\left(N_{i}\right) \subset N_{j}$ for all the arrows. A simple representation is the one whose sub representations are the zero and itself. The quiver representation with dimension vector $(0, \ldots, 1,0, \ldots, 0)$ is a simple representation, here all the linear maps associated with the quiver arrows are trivial. The direct sum of two representations $M \bigoplus N$ consists of the vector space $M_{i} \bigoplus N_{i}$ on each node, and the new linear maps associated with the arrows are $M_{\alpha} \bigoplus N_{\alpha}$. The indecomposable representation is the one which can not be written as the direct sum of two representations. By definition, the simple representation is an indecomposable representation.

Every representations can be decomposed as a direct sum of the indecomposable representations, and indecomposable representations are very important for the BPS counting problem. It is a very difficult problem to find all the indecomposable representations for a given quiver. However, it is possible to find the dimension vectors explicitly due to the Gabriel (Kac) theorem.

Quiver can be classified using the property of the indecomposable representations of the quiver. A quiver is of finite type if and only if the underlying undirect graph is of the ADE Dynkin type, and there are only finite number of indecomposable representations. For a Dynkin quiver Q, the dimension vectors of indecomposable representations do not depend on the orientation of the arrows in $\mathrm{Q}$. A quiver $\mathrm{Q}$ is of tame type if and only if the underlying directed graph is an extended Dynkin graphs of type $\hat{A}$, $\hat{D}, \hat{E}$. The indecomposable representations of finite and tame quiver are in one-to-one correspondence with the positive roots of the corresponding 
root system. All other quivers are called "wild" and the indecomposable representations are related to the roots of the quiver.

Let's review the Gabriel-Kac theorem in some detail. One can define a Euler form on the positive lattice $\Lambda=Z_{+}^{Q_{0}}$ :

$$
\chi(\alpha, \beta)=\sum_{i \in Q_{0}} \alpha_{i} \beta_{i}-\sum_{\rho: i \rightarrow j} \alpha_{i} \beta_{j} .
$$

The Tits form is defined as $T(\alpha)=\chi(\alpha, \alpha)$. The antisymmetric form from the Euler form is defined as

$$
\langle\alpha, \beta\rangle=\chi(\beta, \alpha)-\chi(\alpha, \beta)
$$

Notice that $\left\langle e_{i}, e_{j}\right\rangle$ count the number of arrows from $i$ to $j$ minus the number of arrows from $j$ to $i$ if $e_{i}=(0, \ldots, 1,0, \ldots, 0)$ with one on the $i$ th quiver node.

The roots could be found explicitly just from the structure of the quivers. Let's denote $e_{i}=(0, \ldots, 1,0, \ldots, 0)$ as the simple roots and $I$ as the set of all the simple roots, and define the reflection $s_{i}$ on lattice $Z^{Q_{0}}$ :

$$
s_{i}(d)=d-\left\langle e_{i}, d\right\rangle e_{i}
$$

The Weyl group $W(Q)$ is defined as the subgroup generated by $s_{i}$. The fundamental domain $F(Q)$ is defined as the set of all non-zero dimension vector $d$ with connected support, i.e. the full sub quiver with nonzero $d_{i}$ is connected, such that $\left(e_{i}, d\right) \leq 0$ for all $i \in I$. The set of real roots are

$$
\Delta^{r e}(Q)=W(Q) I
$$

and the set of imaginary roots are

$$
\Delta^{i m}(Q)=W(Q) F(Q)
$$

There exists an indecomposable representation of $Q$ of dimension vector $d$ if and only if $d$ is a positive root $d$,

$$
d=\sum_{i} d_{i} e_{i}, \quad d_{i} \geq 0 .
$$

In case $d \in \Delta^{r e}(Q)$, there exists a unique indecomposable (up to isomorphism) of dimension vector $d$. In case $d \in \Delta^{I m}(Q)$, the number of parameters of the set of indecomposable representations is $1-\chi(d, d)$. For the 
real roots $d$, we have $\chi(d, d)=1$. Alternatively, the positive real roots are the positive integer solution of the following equation $\chi(d, d)=1$, and the positive imaginary roots are the positive integer solutions of the quadratic equation $T(d, d) \leq n$ with $n \leq 0$.

From physics' perspective, the number of parameters of an indecomposable representation is equal to the dimension of the Higgs branch of the quiver gauge theory with gauge group $U\left(d_{i}\right)$ : each arrow contributes $d_{i} d_{j}$ and the dimension of the gauge group is $d_{i}^{2}$, and an overall $U(1)$ is decoupled, so the dimension of the Higgs branch (assume that all the gauge symmetry is broken) is

$$
\operatorname{Dim}(d)=\sum d_{i} d_{j}-\sum_{i} d_{i}^{2}+1=1-\chi(d, d)
$$

Let's now give the identification between the possible BPS particles and the indecomposable representations:

Statement 1. Each indecomposable representation ${ }^{5}$ (up to isomorphism) with dimension vector $d$ represents a possible BPS particle, and the charge vector $\gamma$ of this state is the following

$$
\gamma=\sum d_{i} e_{i}
$$

Here $e_{i}$ is the dimension vector for the simple representation associated with the quiver node, which represent the elementary BPS particle.

Statement 2. The spin of the BPS particle is equal to $1-\chi(d, d)$. The real roots give the hypermultiplet and the imaginary roots give the higher spin states.

3.1.1. Stability condition and stable BPS particle. After identifying the possible BPS states with the indecomposable representation, it is time to study the stability condition from which one can judge whether an indecomposable representation is stable or not. The $\theta$ stability condition on quiver representation is the one which we are going to use. Let's briefly review those concepts below.

\footnotetext{
${ }^{5}$ More precisely it is the Shur representation whose endmorphism is $\operatorname{End}(V)=C$.
} 
Let's fix the dimension vector $d_{i}$ and denote the complex vector space at each quiver node as $M_{i}$. Consider the following space

$$
R_{d}=\bigoplus_{\alpha} \operatorname{Hom}\left(M_{i}, M_{j}\right),
$$

obviously each point of $R_{d}$ parametrizes a representation. The linear group $G_{d}=\prod_{i} G L\left(M_{i}\right)$ acts on $R_{d}$ via the following gauge transformation on an element $M_{a} \in \operatorname{Hom}\left(M_{i}, M_{j}\right)$ :

$$
M_{a} \rightarrow g_{j} M_{a} g_{i}^{-1} .
$$

Therefore each $G_{d}$ orbit parametrizes an isomorphism class of the quiver representation with dimension vector $d$. Physically each orbit of $G_{d}$ parametrizes all the gauge equivalent scalar field configuration (the gauge group action is the complex one). The moduli space $R_{d} / G_{d}$ parameterizes all the representations with dimension vector $d$, however, this space is very complicated. The space is much more simpler if we concentrate on a subspace which covers almost all the quiver representation, this is the place where the stability condition plays an important role.

The stability condition (central charge) $Z(d)$ for the quiver representation is a linear functional acting on the lattice of dimension vectors which is generated by $Z\left(e_{i}\right)$ defined on each vertex. Then for a representation with nonnegative dimension vectors $d$, the central charge is

$$
Z(d)=\sum_{i} d_{i} Z\left(e_{i}\right)
$$

The slop of a representation is defined as $\mu(d)=\arg Z(d){ }^{6}$, and the definition of the slop depends only on the dimension vector but not on the quiver arrows. The quiver information enters into the characterization of the stability condition though. We say a representation semistable (reps. stable) if for any proper subrepresentation ${ }^{7} N$, we have $\mu(N) \geq \mu(M)$ (resp. $(\mu(N)>\mu(M))$. All the simple representations are stable since it has no proper subrepresentation, and all the stable representations are indecomposable, which can be seen as follows: if a representation $P$ is decomposable

\footnotetext{
${ }^{6}$ Usually the slop for a representation is defined as $\mu_{\theta}(d)=\frac{\theta_{i} d_{i}}{\sum d_{i}}$, where $\theta_{i}$ is defined on each quiver node, and this gives the name for the $\theta$ stability. Our definition is slightly different but actually equivalent.

${ }^{7}$ The zero representation and the representation itself is not included into the proper subrepresentation.
} 
as $P=\sum_{i} M_{i}$ in which $M_{i}$ is the subrepresentation of $M$, and the dimension vector is decomposed as $d=d_{1}+d_{2}+\cdots+d_{s}$, then there is at least one of representation say $M$ whose slop is bigger than $P$, which implies the representation $P$ is unstable.

We denote $M_{\theta}^{s s}(Q, d)\left(\operatorname{resp} M_{\theta}^{s}(Q, d)\right)$ as the moduli space of semistable (reps. stable) representations. It was shown in [48] that each point in $M_{\theta}^{s}(Q, d)$ parametrizes a solution to the $D$ term equations modulo complex gauge group transformation of the quiver gauge theory:

$$
\sum_{a: i \rightarrow *} \phi^{a+} \phi^{a}-\sum_{a: * \rightarrow i} \phi^{a} \phi^{a+}=\theta_{i} I_{d_{i}},
$$

here $\theta_{i}$ is related to the stability condition, so $\theta_{i}$ is just the Fayet-Iliopolous (FI) term for the quiver gauge theory defined using the dimension vector $d$. $M_{\theta}^{s}(Q, d)$ simply parameterizes the moduli space of the gauge theory with FI terms turned on.

Now we are coming to the third identification of the BPS spectrum and quiver representation from the $\theta$ stability condition:

Statement 3. The $\theta$ stability condition is the stability condition ${ }^{8}$ for the BPS particles: the stable indecomposable representation represents the stable BPS particle.

One can immediately derive some general features about the BPS spectrum: first, there are at least $n$ stable BPS particle since every simple representation associated with the quiver node is stable regardless of the chosen stability condition; second, if there is a BPS particle with charge $\gamma$, then $k \gamma$ with $k \geq 2$ can not be the charge vectors for the BPS particle.

If we change the stability condition, and some of the stable representations would become unstable, which leads to the wall crossing behavior. The following two examples are very suggestive to us. Consider the $A_{2}$ quiver which is the BPS quiver for $\left(A_{1}, A_{2}\right)$ Argyres-Douglas theory:

The simple representations $S_{1}$ and $S_{2}$ corresponding to two quiver nodes are indecomposable. The only other indecomposable representation $P$ is

$$
1 \stackrel{1}{\rightarrow} 1
$$

\footnotetext{
${ }^{8}$ More precisely, we only consider the discrete stability condition in this paper, which means that only one BPS particle is allowed for each slop.
} 
whose only subrepresentation is $S_{2}$. If the stability condition is chosen such that $\arg \left(Z\left(S_{1}\right)\right)>\arg \left(Z\left(S_{2}\right)\right)$, then $P$ is not stable, and there are only two stable BPS particles correspond to $S_{1}$ and $S_{2}$. If the stability condition is taken such that $\arg \left(Z\left(S_{1}\right)\right)<\arg \left(Z\left(S_{2}\right)\right)$, then $P$ is stable and we have three stable BPS particles. This exhausts the two possible chambers for this theory.
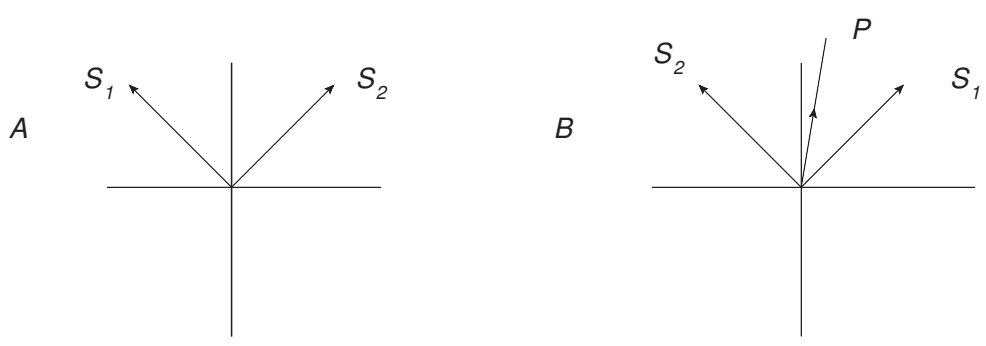

Figure 7: The two chambers for $A_{2}$ quiver depending on different choices of the stability condition.

Let's consider the following affine $A_{1}$ quiver which is the BPS quiver for pure $S U(2)$ SYM:

$$
\bullet \bullet
$$

The real roots for this quiver are $e_{1}$ and $e_{2}, f_{n}=(n, n+1)$ and $d_{n}=(n, n-$ 1 ). Unlike the previous examples, this quiver has an imaginary root $Q=$ $(1,1)$ which represents the $\mathrm{W}$ boson. These are all possible indecomposable representations for this quiver, and $e_{2}$ is the subrepresentation of $d_{n}, f_{n}$ and $Q$.

If the stability conditions are taken such that $\arg \left(Z\left(S_{1}\right)\right)>\arg \left(Z\left(S_{2}\right)\right)$, then $S_{1}$ and $S_{2}$ corresponding to roots $e_{1}$ and $e_{2}$ are the only stable representations. If $\arg \left(Z\left(S_{1}\right)\right)<\arg \left(Z\left(S_{2}\right)\right)$, then all the indecomposable representations are stable! These two chambers also successfully recover the BPS spectrum of the two chambers of the pure $S U(2)$ theory.

This method can be generalized to the other simple ADE quivers, however, it would be a formidable problem for very complicated BPS quiver of general $\mathcal{N}=2$ theory. In next section, we are going to use other combinatorial methods to deal with the BPS counting problem, but the quiver representation theory is always conceptually useful.

3.1.2. Quantum dilogarithm identity. If there are two stability conditions and two different BPS chamber, then what is the invariant constructed from them? Kontestvich-Soilbeman wall crossing formula provides 

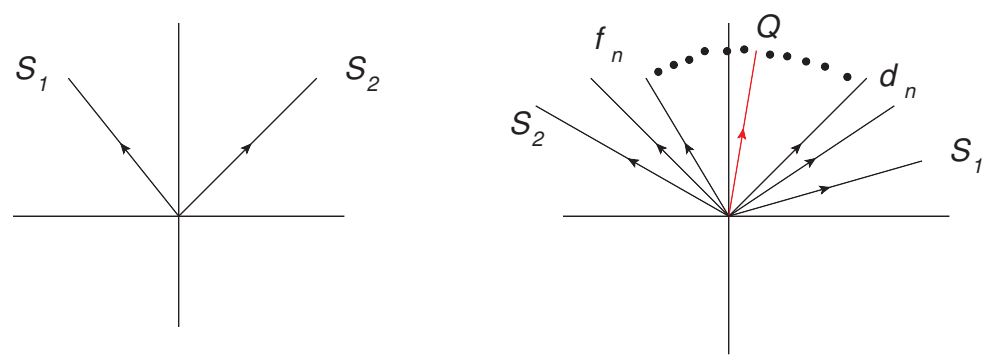

Figure 8.

an invariant, and Reneke derived the similar formula using the Hall algebra and Harder-Narasimhan filtration [47], see also the exploration of the quiver invariant and the BPS spectrum in physics literature [49-53].

Reneke's construction starts with a quantum algebra on the lattice $Z_{+}^{Q_{0}}$ for the quiver: one associate a quantum operator to each dimension vector, and they satisfy the condition:

$$
y^{\alpha} y^{\beta}=q^{\frac{1}{2}\langle\alpha, \beta\rangle} y^{\alpha+\beta},
$$

here $\langle\alpha, \beta\rangle$ is the antisymmetric form we defined earlier.

The construction of the invariant uses the Hall algebra and HarderNarasimhan filtration in an essential way. Let's first review the HarderNarasimhan filtration: a filtration $0=X_{0} \subset X_{1} \subset \cdots \subset X_{s}=X$ of a representation is called Harder-Narasimhan (HN) if all the sub quotients $X_{i} / X_{i-1}$ is semistable and $\mu\left(X_{1} / X_{0}\right)>\mu\left(X_{2} / X_{1}\right)>\cdots>\mu\left(X_{s} / X_{s-1}\right)$. Every representation $X$ poses a unique HN filtration.

A Hall algebra can be defined on the isomorphic classes of quiver representations

$$
[M] \cdot[N]=\sum_{[X]} F_{M, N}^{X} \cdot[X]
$$

where $F_{M, N}^{X}$ denotes the number of sub representations $U$ of $\mathrm{X}$ which are isomorphic to $N$, with the quotient $X / U$ isomorphic to $M$. This coefficient is finite and the sum is also finite, and he dimension of $[X]$ is equal to the sum of dimension $[M]$ and $[N]$. Let's consider the following special elements in the Hall algebra

$$
e_{d}=\sum_{\operatorname{dim} M=d}[M], \quad e_{d}^{s s t}=\sum_{\operatorname{dim} M=d}^{M \text { semistable }}[M],
$$


here $e_{d}$ contains all the quiver representation isomorphism class with dimension vector $d$, and $e_{d}^{s s t}$ contains all the semi-stable isomorphism class with dimension vector $d$. Now because of the uniqueness of HN filtration, $[M]$ appears with coefficient 1 in the product

$$
\prod e_{d_{s}}^{s s t} \cdots e_{d_{2}}^{s s t} e_{d_{1}}^{s s t}
$$

and we have

$$
e_{d}=\sum_{*} e_{d_{s}}^{s s t} \cdots e_{d_{1}}^{s s t},
$$

where the sum is running over all decompositions $d_{1}+\cdots+d_{s}=d$ such that $\mu\left(d_{1}\right)>\cdots>\mu\left(d_{s}\right)$. In particular, we have the following identity

$$
\sum_{d} e_{d}=\prod_{\rightarrow} 1_{\mu}^{s s t}
$$

where the right rand side is the ordered product on the semistable quiver representations, and $1_{\mu}^{s s t}=1+e_{\mu}^{s s t}$. The left-hand side does not depend on the stability condition, so this identify shows that there is an invariant for each stability condition.

Now use the evaluation map which maps an element from the Hall algebra to the power series in torus algebra

$$
[M] \rightarrow \frac{(q)^{1 / 2 \chi(\alpha, \alpha)}}{\prod\left(q^{-1}\right)_{\alpha_{i}}} y^{\alpha}
$$

where $\left(q^{-1}\right)_{n}=\prod_{i=1}^{n}\left(1-q^{-i}\right)$.

Let's consider the case where all the stable representation correspond to the real roots, which implies $\chi(\alpha, \alpha)=-1$. If $M$ is semistable, then all the power $M^{n}$ would be also semistable and the charge vectors of $M^{n}$ is $n \alpha$ whose Tits form is $\chi(n \alpha, n \alpha)=-n^{2}$. Therefore, for a single stable representation, we have the following series in the torus algebra

$$
\left[1+\sum e_{\mu}^{s s t}\right]=1+\frac{q^{-1 / 2}}{\prod\left(q^{-1}\right)_{\alpha_{i}}} y^{\alpha}+\cdots+\frac{q^{-n^{2} / 2}}{\prod\left(q^{-1}\right)_{n \alpha_{i}}} y^{n \alpha}+\cdots
$$

Let's consider $\alpha$ as the simple representation associated with a quiver node, then $\alpha=(0, \ldots, 1, \ldots, 0)$ and $y^{\alpha}=y_{i}$; the above series becomes

$$
E\left(y_{i}\right)=1+\frac{q^{\frac{1}{2}}}{q-1} y_{i}+\cdots+\frac{q^{n^{2} / 2} y_{i}^{n}}{\left(q^{n}-1\right)\left(q^{n}-q\right) \cdots\left(q^{n}-q^{n-1}\right)}+\cdots
$$


which is the famous quantum dilogarithm function. In general for an stable indecomposable representation from the real root $\alpha$, one can associate a quantum dilogarithm function $E\left(y^{\alpha}\right)$, and the identity (46) implies that

$$
E\left(y^{\alpha_{1}}\right) \cdots E\left(y^{\alpha_{s}}\right)=E\left(y^{\beta_{1}}\right) \cdots E\left(y^{\beta_{r}}\right)
$$

and the product is taken over the stable representation with the decreasing order of the slop, here $\left(\alpha_{1}, \ldots, \alpha_{s}\right)$ are the stable representations of on stability condition, and $\left(\beta_{1}, \ldots, \beta_{r}\right)$ are the stable representations of the other stability condition. One could write a similar formula for the stability condition involving higher spin states.

\subsection{Quiver with potential}

Remember that the BPS quiver for a theory is really a class of quivers related by the quiver mutations. The quiver representation theory is definitely very different for the quivers related by the quiver mutations. For instance, consider the $A_{3}$ quiver and the affine $\tilde{A}(3,0)$ quiver which are related by quiver mutations, see Figure 9 . There are only finite number of indecomposable representations for the $A_{3}$ quiver, but there are infinite many for affine $\tilde{A}(3,0)$ quiver.
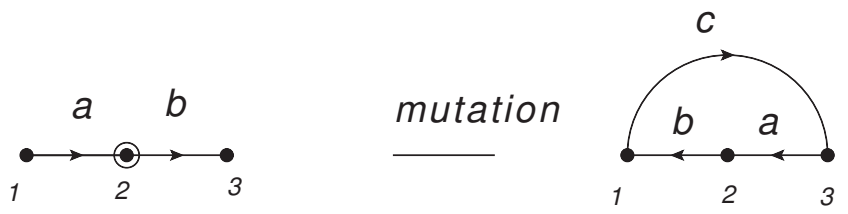

Figure 9: $A_{3}$ quiver and affine $A_{2}$ quiver which are related by quiver mutations.

To solve this problem, one need to add constraints to the affine quiver to kill many representations. In the original context of Seiberg duality, to match the moduli space, Seiberg introduced a new superpotential term to the quiver. In the same spirit, the addition of the potential will kill these extra representations. In fact, it is possible to add a unique superpotential $W$ term to the affine $\tilde{A}(3,0)$ quiver. Now a representation of the quiver with potential should satisfy the extra condition

$$
\frac{\partial W}{\partial \phi_{i}}=0
$$


for all the fields $\phi_{i}$ appearing in the potential. The general analysis of the indecomposable representations of the quiver with potential is quite complicated, and we would like to introduce an algebraic approach.

To deal with this case, let's first introduce the path algebra associated with a quiver. The path algebra $C Q$ is generated by the quiver arrows, moreover we need to add the length zero generator $e_{i}$ attached on the quiver node, so the elements for the path algebra are

- Path $\phi_{i j}$ in the quiver going from $i$ to $j$.

- The length zero element $e_{i}$.

The product between two elements in the path algebra is very simple: the only nonzero products are $\phi_{i j} \phi_{j k}, e_{i} \phi_{i j}=\phi_{i j}, e_{i}^{2}=e_{i}$. For example, for the quiver in left of Figure 9, the path algebra has elements

$$
\left[e_{1}, e_{2}, e_{3}, a, b, a b\right]
$$

The path algebra is finite if and only if there is no oriented cycle in the quiver. The nice thing about the path algebra is that the category of quiver representation is the same as the category of $C Q$ left modules ${ }^{9}$. One could also define the direct sum of two modules, submodule, and the indecomposable modules in a similar way as we did for the quiver representation theory. Similarly, one can define the stability conditions, etc. The special module $P_{i}$ attached to a vertex plays an important role, here $P_{i}=(C Q) e_{i}$ consists all the paths ending at node $i$. The nice thing about the $P_{i}$ module is that: the $P_{i}$ are projective modules, and every projective module is a direct sum of $P_{i}$. The dimension of the homomorphism $\operatorname{Hom}\left(P_{i}, P_{j}\right)$ are the number of independent paths from node $i$ to node $j . P_{i}$ is the left-module representing the indecomposable representation corresponding to the simple root.

Now if we add a potential to the quiver, the path algebra is modified and becomes the so-called Jacobi algebra. The potential will give zero relations which will kill some of the generators in the original path algebra. For example, consider the quiver on the right of Figure 9 in which there is a

${ }^{9}$ A left CQ-modulue consists an an abelian group $(M,+)$ and operation $C Q \times$ $M \rightarrow M$ such that for all $r, s$ in $C Q$ and $x, y$ in $M$, we have following condition

$$
\begin{array}{r}
\text { 1. } r(x+y)=r x+r y \\
\text { 2. }(r+s) x=r x+s x \\
\text { 3. }(r s) x=r(s x)
\end{array}
$$

4. $1 . s=s$ 
potential term $W=a b c$, and the $F$ term equation from the potential would be

$$
a b=0, \quad b c=0, \quad c a=0 .
$$

Now the Jacobi algebra is generated by

$$
\left[e_{1}, e_{2}, e_{3}, a, b, c\right]
$$

and it is finite dimensional. The rule for the product in the algebra is the same as the one defined for the path algebra, and they should satisfy the relation in doing the product (54), say $a b=0$, etc. Notice that although the two quivers in Figure 9 are related by quiver mutation, the two Jacobi algebra is not equivalent, which is natural since the quiver representation theory is not the same even with the inclusion of the potential. They however define the same quiver invariant which is formed from the BPS spectrum.

After defining the Jacobi algebra, one can similarly define the modules, direct sum of modules, and indecomposable modules, etc; Now the possible BPS states are represented by the indecomposable module of the Jacobi algebra, and the stability conditions can be similarly defined, everything is kind of similar. The mathematical results about the representation theory of quiver with potential is fruitful and they play an important role in the studying of the BPS spectrum and wall crossing, see $[8,10,54,55]$. However, the mathematical descriptions are quite complicated and we do not really use these descriptions in our later description, so we will not discuss them in details and leave it to other occasions in the future.

\section{Hypermultiplets: Maximal green mutation}

\subsection{Maximal green mutation: Definition}

The detailed analysis of the quiver representation theory is rather complicated, especially when the nontrivial potential exists. There is a remarkable combinatorial method called green mutation [37] which will make the task of finding the finite chamber much easier.

Let's first modify the quiver as follows: introduce an extra frozen node ${ }^{10}$ for each quiver node, and there is a quiver arrow pointing into the frozen

\footnotetext{
${ }^{10} \mathrm{~A}$ frozen node can never be mutated, which could be though of as the flavor group.
} 
node, see Figure 10A. The original quiver and the extended quiver are denoted as $Q$ and $\tilde{Q}$ respectively. A non frozen node is called green if it is the source to the frozen nodes, and called red if it is the sink to the frozen nodes. A green mutation sequence is the one where only green nodes can be mutated, and the mutation rule for the extended quiver is the same as the ordinary one. Several features of the green mutation is immediately clear from the definition:

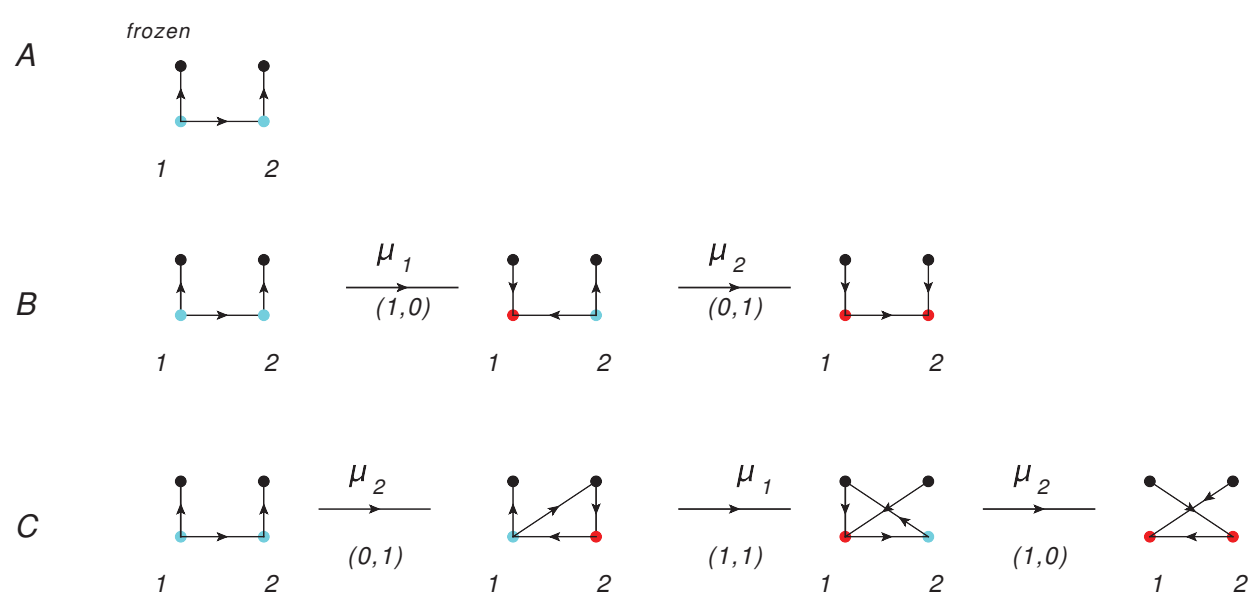

Figure 10: A: The extended quiver derived by adding extra frozen nodes. B: A maximal green mutation sequences. C: Another maximal green mutation sequences of the same quiver.

- If we assign the charge vector $\gamma_{i}$ on each quiver node, and the Dirac product of these charges satisfies the condition

$$
\left\langle\gamma_{i}, \gamma_{j}\right\rangle=\epsilon_{i j}
$$

(this can be done explicitly by taking $\gamma_{i}=(0, \ldots, 1, \ldots, 0)$ ). The charge vector of the quiver node during the quiver mutation is determined by the quiver arrows connected with the frozen nodes, i.e. the charge vector is

$$
\lambda=\sum m_{i} \lambda_{i}
$$

where $m_{i}$ is positive (resp. negative) if the quiver node is the source (resp. sink) for the frozen nodes. For the green node, the subquiver formed by positive $m_{i}$ is connected. Let's denote the charge vector as $\alpha$, then after the 
mutation on node $k$, the new charge vector is changed as follows:

$$
\begin{aligned}
& \alpha_{k}^{\prime}=-\alpha_{k}, \\
& \alpha_{i}^{\prime}=\alpha_{i}+\left\langle\alpha_{i}, \alpha_{k}\right\rangle \alpha_{k} \quad \text { if }\left\langle\alpha_{i}, \alpha_{k}\right\rangle 0,
\end{aligned}
$$

this formula can be checked by looking at the green mutation. The new quiver is formed by doing the Dirac product using $\alpha^{\prime}$.

- Each node is either green or red at any step of the green mutation. This can be seen as follows: let's mutate a green node $k$, by the rule of mutation, all the quiver arrows including the arrows to the frozen node are reversed, then it becomes red after the mutation. The color of the other quiver nodes $i$ would not change if there is no quiver arrow to node $k$ or $i$ is the sink of the arrows between $i$ and $k$. If $i$ is the source of the arrows between $i$ and $k$, then we have two choices to consider: A. $i$ is green, then $i$ is also green after the mutation; B. $i$ is red, this case is a little bit complicated, but it can be proven that it is either green or red after we identify the charge vector as the $c$ vector appearing in the study of the cluster algebra. So we have the following conclusion: The green node is still green after a green mutation on other nodes, and the red node can become either red or green.

- If one green node with charge vector $m_{i}$ is mutated, the quiver moduli space with the assignment of dimensions $m_{i}$ has dimension zero, and therefore this corresponds to a hypermultiplet!

Now let's introduce the definition of maximal green mutation sequence:

Definition 1. A maximal green mutation sequence is the finite green mutation sequence such that all the nodes are red at the end. It is not hard to see that the final quiver has basis $-\gamma_{1},-\gamma_{2}, \ldots$, and the quiver (non frozen part) is isomorphic to the original quiver.

Now each maximal green mutation sequence represents a chamber with finite number of (hypermultiplets) states! This method is essentially equivalent to that proposed in [21] ${ }^{11}$, but the combinatorial way presented here make the calculation much easier. An example of maximal green mutations is shown in Figure 10B, and this chamber has two BPS particles with charge $\gamma_{1}$ and $\gamma_{2}$.

\footnotetext{
${ }^{11}$ In that paper, one only mutate the quiver nodes with positive coefficient of the original charges, which means only mutating the green node in our language, moreover, each mutation corresponds to rotating the half-plane of the central charges, and when the plane is rotated by 180 degree, the canonical basis is $-\gamma_{i}$, which essentially means that no green node is left.
} 
In the above procedure, we have fixed a quiver from the very beginning, now according to our previous discussion on the BPS quiver, all the quivers appearing in this sequence are equally good for describing this particular BPS chamber, see the illustration in Figure 11. Let's denote this subset as $[Q]_{G}$, apparently only a subset of the quivers in the mutation equivalence class would appear, and this subset depends on a particular maximal green mutation sequence. Different green mutation sequence defines different subsets. If a quiver appears in $[Q]_{G}$, then there will be a maximal green mutation sequence with length $|G|$.

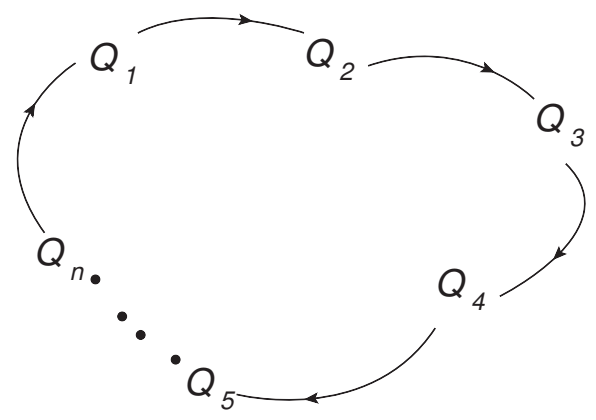

Figure 11: The quivers connected by the green mutation.

There are several observations about the feature of maximal green mutations:

1. The length of the maximal green mutations is at least $|Q|$, where $|Q|$ is the number of quiver nodes, and each node is mutated at least once. If there is a BPS particle with charge $\alpha_{i}$ in the spectrum, then the charge vector $k \alpha_{i}, k \geq 2$ will not appear. Notice that this is consistent with the quiver representation theory by taking discrete stability condition.

2. The final quiver is isomorphic to the original quiver, and each red node is only connected to one frozen node.

Special quiver. Let's consider a bipartite quiver which has only two types of quiver nodes: source and sink (see Figure 12), and the following two special mutation sequences

$$
\begin{array}{ll}
\mu_{+}=\mu_{i_{1}} \cdots \mu_{i_{m}}, & i_{k} \text { is the source node } \\
\mu_{-}=\mu_{j_{1}} \cdots \mu_{j_{n}}, & j_{k} \text { is the sink node }
\end{array}
$$

The order of mutations in $\mu_{+}$and $\mu_{-}$does not matter since there are no arrows between the source nodes (the same is true for the sink nodes). Due 
to the special structure of the quiver, there is no cycle in the quiver and therefore no potential is allowed. The mutation sequences $\tau=\mu_{+}^{2}$ is a maximal green mutation sequence as can be easily checked from the definition.



Figure 12: The bipartite quiver has two types of quiver nodes: sink and source nodes.

The above observation can be generalized to an acyclic quiver which always has at least a source node. Let's do the green mutation on this source node, and the new quiver is still acyclic and there is again a source node. Continuing mutating the source node, we are going to find the maximal mutation sequence with $|Q|$ steps.

The above mutation method only gives us the hypermultiplet. The vector multiplet does not correspond to the quiver mutation, but it can be taken as limit of a infinite number quiver mutation sequence. If there is only one vector multiplet, one might be able to probe the existence of the vector multiplet by doing the maximal red mutation: one add a frozen node and quiver arrow for each quiver node, but the arrow is pointing into the quiver node. The red mutation sequence is the one which only red node is mutated. The interested reader can check that the red mutation has quite similar property as green mutations. This red mutation sequence corresponds to rotating the half plane in counterclockwise direction. The infinite chamber with one vector multiplet can found as follows: first do the maximal green mutation and then do the red mutation carefully to make everything consistent. If these two sequences has a common limit, then we conclude that there is a vector multiplet. 


\subsection{Finding maximal green mutation sequence: a clue}

There is one serious question about the use of the maximal green mutation: there is no information on the order of green mutations and random green mutations usually would not stop at finite steps. The quiver representation theory and $\theta$ stability do tell us something about the green mutation sequences, we have the following conjecture:

Conjecture 2. There are infinite number of BPS states if there are stable higher spin states.

According to this conjecture, the stability condition should be chosen such that no higher spin stable state exists. For simplicity let's assume that the vector multiplet is the possible higher spin states, and denote the corresponding representation as $V_{d}$, and its subrepresentation as $V_{1}, V_{2}, \ldots, V_{s}$. According to the $\theta$ stability, $V_{d}$ is stable if and only if its slop is smaller than all of its sub representations:

$$
\mu\left(V_{i}\right)>\mu\left(V_{d}\right), \quad \text { for all } i,
$$

So to have a finite chamber, we must ensure that such situation will not happen in our mutation process.

Let's look at an example to see how to use the stability condition to determine maximal green mutation sequences. The BPS quiver is the one representing $S U(2)$ theory with one flavor, see Figure 13 . The gauge boson corresponds to the representation $d=(1,1,1)$, and $S_{2}=(0,1,0), P=(1,1,0)$ are the sub representations of it. Now, let's start doing mutation on node 2 in step 1, and probe a BPS particle with charge $S_{2}$ on the far left. The node one and node three are green nodes now. In this second step, if mutate node 1 which has charge $\gamma_{1}+\gamma_{2}$ and probe the BPS particle $P$, then the vector multiplet is definitely stable since it would have smaller slop than $S_{2}$ and $P$. So to find a finite chamber, we can only mutate node 1 . In step 3 , similar analysis forces us to mutate node 2 , etc. At the end, we find the following maximal mutation sequences

$$
\mu_{2}, \mu_{3}, \mu_{2}, \mu_{1}, \mu_{3} .
$$

Similar analysis can be done on the mutation sequences starting from other nodes. A simple but useful fact is that if in the mutated quiver $Q_{1}$, and there are two green nodes connected by double arrows, then we can not mutate the sink nodes of this subquiver which follows directly from the representation 
theory of affine $A_{1}$ quiver. This follows from the fact the mutated quiver is equally good for describing this particular chamber. Use this observation, it is easy to see that in step 3 , we can only mutate quiver node 2 .

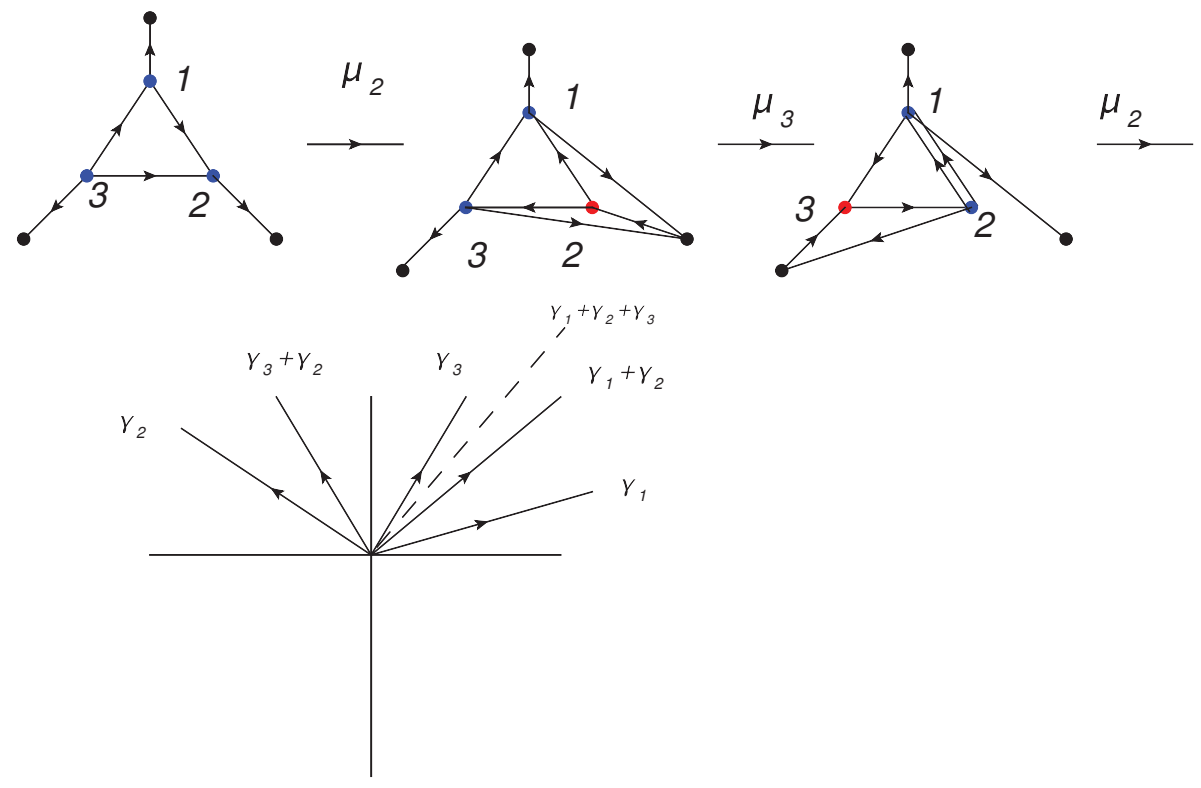

Figure 13: Up: the maximal green mutation sequences. Bottom: the charge vector for the various BPS particle, the dash line represent the vector boson which is not stable, since the slop of its subrepresentation $\gamma_{1}+\gamma_{2}$ is smaller than it.

\subsection{Wall crossing: Quantum dilogarithm identity}

Usually there are more than one maximal green mutation sequences for a given quiver. It would be nice to have an invariant built from the BPS spectrum, which can be written down using the quiver representation theory. For the finite chamber, it is actually very easy to write the invariant using the maximal green mutation data as shown in [37]. Let's fix a quiver and consider the quantum algebra on charge lattice, and the commutation relation is

$$
X^{\alpha} X^{\beta}=q^{\frac{1}{2}\langle\alpha, \beta\rangle} X^{\alpha+\beta},
$$

where $\langle\alpha, \beta\rangle$ is the familiar Dirac product.

Assume that we find a maximal green mutation sequence $\mathbf{k}=\left(k_{1}, k_{2}, \ldots\right.$, $k_{s}$ ) and the charge vector in step $i$ is $\alpha_{i}$, we can form a quantum dilogarithm 
function for $i$ th mutation:

$$
E\left(X^{\alpha_{i}}\right)
$$

Given a maximal green mutation sequences, we can form a quantum dilogarithm product

$$
E(k)=E\left(X^{\alpha_{1}}\right) E\left(X^{\alpha_{2}}\right) \cdots E\left(X^{\alpha_{s}}\right),
$$

If there is another maximal green mutation sequences $k^{\prime}$ whose length is $r$, then this represents another chamber and we can form another quantum dilogarithm product $E\left(k^{\prime}\right)$, then these two quantum dilogarithm products are the same

$$
E(k)=E\left(k^{\prime}\right) .
$$

which could be interpreted as the wall crossing formula, which has been proved using quiver representation theory.

Example. Let's consider our familiar $A_{2} \mathrm{AD}$ theory. There are two maximal green mutation as shown in Figure 10. The first chamber has two BPS hypermultiplets with charges $\gamma_{1}, \gamma_{2}$, and the other chamber has three BPS hypermultiplets with charge $\gamma_{2}, \gamma_{1}+\gamma_{2}, \gamma_{1}$ (the charges are listed in the order of decreasing phase angle). The quantum dilogarithm identity from this theory is

$$
E\left(X^{\gamma_{1}}\right) E\left(X^{\gamma_{2}}\right)=E\left(X^{\gamma_{2}}\right) E\left(X^{\gamma_{1}+\gamma_{2}}\right) E\left(X^{\gamma_{1}}\right) .
$$

The above identity has the familiar form if we use the generator $X_{i}=X^{\gamma_{i}}$ :

$$
E\left(X_{1}\right) E\left(X_{2}\right)=E\left(X_{2}\right) E\left(q^{-\frac{1}{2}} X_{1} X_{2}\right) E\left(X_{1}\right) .
$$

\subsection{Quantum dilogarithm identity from quantum cluster algebra}

In this part, a proof of the quantum dilogarithm identity is given using the quantum cluster algebra.

4.4.1. Charge vector as $c$ vector. The charge vector appeared in previous section has a nice interpretation from the cluster algebra. Let's first introduce some background on tropical semi-field. Assume the semi field 
is generated by generators $y_{i}, i=1, \ldots, n$, and each element has the form $\prod y_{i}^{a_{i}}$, where $a_{i}$ is an integer. The tropical sum is defined as

$$
\prod y_{i}^{a_{i}}+\prod y_{i}^{b_{i}}=\prod y_{i}^{\min \left(a_{i}, b_{i}\right)} .
$$

Given a quiver and consider the mutation rule of the cluster $X$ coordinates,

$$
\begin{aligned}
& X_{k}^{\prime}=X_{k}^{-1} \\
& X_{i}^{\prime}=X_{i}\left(1+X_{k}^{-\operatorname{sgn}\left(\epsilon_{i k}\right)}\right)^{-\epsilon_{i k}} .
\end{aligned}
$$

If we replace the ordinary sum in the new $X$ variables with the tropical sum, then the cluster coordinates has the following form

$$
[X]=\prod X_{i}^{c_{i}}
$$

where $c_{i}$ is an integer vector whose entries are all nonpositive or nonnegative [56], and $X_{i}$ is the original cluster variable. These $c$ vectors are not the new stuff, and they are just the charge vector appearing in the study of green mutation. The initial $c$ vector has only one entry 1 with all the other entries zero, and the final $c$ vector has only one entry -1 , see an example in Figure 14.
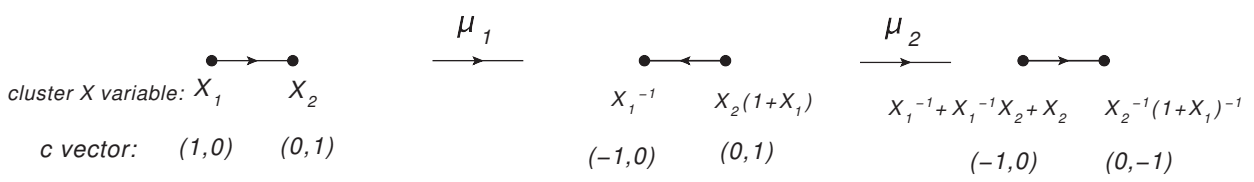

Figure 14: The $X$ variable and $c$ vectors of the mutations.

The $c$ vector has the following simple transformation rule from the definition if a mutation is done on the vertex $k$ :

$$
\begin{aligned}
& c_{k}^{\prime}=-c_{k} \\
& c_{i}^{\prime}=c_{i} \quad \text { if } \epsilon_{i k} c_{k} \leq 0 \\
& c_{i}^{\prime}=c_{i}+\epsilon_{i k} c_{k} \quad \text { if } \epsilon_{i k} c_{k}>0
\end{aligned}
$$


Since we always mutate on green nodes which means the $\operatorname{sign}\left(c_{k}\right)$ is always positive, the above formula simplifies as

$$
\begin{aligned}
& c_{k}^{\prime}=-c_{k} \\
& c_{i}^{\prime}=c_{i} \quad \text { if } \epsilon_{i k}<0 \\
& c_{i}^{\prime}=c_{i}+\epsilon_{i k} c_{k} \quad \text { if } \epsilon_{i k}>0
\end{aligned}
$$

Let's now use the $c$ vector analysis to prove the maximal green sequences for the bipartite quiver. Let's consider one of the source node whose initial c vector is $(1,0, \ldots, 0)$, after the mutation $\mu_{+}, c^{\prime}=(-1,0, \ldots, 0)$. The $c$ vector of the sink nodes is not changed. After mutation $\mu_{-}$on the sink node, all the $c$ vector of the sink nodes becomes $c^{\prime}=(0, \ldots,-1,0)$, and the final quiver is a quiver whose nodes are all red.

4.4.2. Quantum cluster algebra. We have actually seen the deformations in defining a quantum torus algebra on the charge lattice, and the cluster algebra has a quantum deformation too $[57,58]$, here we only use the quantum version of the cluster $X$ variable, and keep the $A$ variable as classical. There is a quantum $X$ variable associated with each vertex and the noncommutative relations are

$$
X_{i} X_{j}=q^{\epsilon_{i j}} X_{i} X_{j}
$$

and the transformation rules for the quantum cluster variables under the mutation on node $k$ are

$$
\begin{aligned}
& X_{k} \rightarrow X_{k}^{-1} \\
& X_{i} \rightarrow X_{i}\left(\prod_{a=1}^{\left|\epsilon_{i k}\right|}\left(1+q^{a-1 / 2} X_{k}^{-\operatorname{sgn}\left(\epsilon_{i k}\right)}\right)\right)^{-\operatorname{sgn}\left(\epsilon_{i k}\right)},
\end{aligned}
$$

this transformation preserves the form of the commutation relation $X_{\alpha}^{\prime} X_{\beta}^{\prime}=$ $q^{\epsilon_{\alpha \beta}^{\prime}} X_{\beta}^{\prime} X_{\alpha}^{\prime}$. The mutation can be decomposed into two steps $\mu_{k}=\mu_{k}^{\prime} * \tau_{k,+}$, where $\tau_{k,+}$ is defined as the transformation

$$
\begin{aligned}
& X_{k}^{\prime}=X_{k}^{-1} \\
& X_{i}^{\prime}=q^{1 / 2 \epsilon_{i k}\left[\epsilon_{i k}\right]_{+}} X_{i} X_{k}^{\left[\epsilon_{i k}\right]_{+}} \text {if } i \neq k .
\end{aligned}
$$


Here $\left[\epsilon_{i k}\right]_{+}=\max \left(0, \epsilon_{i k}\right)$, and the transformation $\mu_{k}^{\prime}$ is given by the adjoint action $\operatorname{Ad}\left(\Psi_{q}\left(X_{k}\right)\right)$ :

$$
\begin{aligned}
X_{i}^{\prime} & =A d\left(\Psi_{q}\left(X_{k}\right)\right) X_{i}=\Psi_{q}\left(X_{k}\right) X_{i}^{\prime} \Psi_{q}\left(X_{k}\right)^{-1} \\
& =X_{i} \Psi_{q}\left(q^{\epsilon_{i k}} X_{k}\right) \Psi_{q}\left(X_{k}\right)^{-1} \\
& =X_{i}\left(\prod_{a=1}^{\left|\epsilon_{i k}\right|}\left(1+q^{-\operatorname{sgn}\left(\epsilon_{i k}\right)(a-1 / 2)} X_{k}\right)\right)^{\epsilon_{i k}} .
\end{aligned}
$$

here $\Psi_{q}$ is the familiar quantum dilogarithm function. What is important is that there are another kind of decomposition using $\mu_{k,-}^{\prime}$ and $\tau_{k,-}$, where $\tau_{k,-}$ is defined by replacing $\epsilon_{i k}$ by $-\epsilon_{i k}$, and $\mu_{k,-}^{\prime}$ is defined by using the adjoint action

$$
\begin{aligned}
A d\left(\Psi_{q}\left(X_{k}^{-1}\right)^{-1}\right) X_{i} & =\Psi_{q}\left(X_{k}^{-1}\right)^{-1} X_{i}^{\prime} \Psi_{q}\left(X_{k}^{-1}\right) \\
& =X_{i}\left(\prod_{a=1}^{\left|\epsilon_{i k}\right|}\left(1+q^{\operatorname{sgn}\left(\epsilon_{i k}\right)(a-1 / 2)} X_{k}^{-1}\right)\right)^{-\epsilon_{i k}}
\end{aligned}
$$

It can be checked that $\mu_{k,-}^{\prime} * \tau_{k,-}=\mu_{k,+}^{\prime} * \tau_{k,+}$.

Here comes the crucial point: if there is a sequence of mutations $\left(\mu_{1}, \mu_{2}\right.$, $\left.\ldots, \mu_{s}\right)$ such that the final cluster coordinates are the same to the original one up to the permutation, there is a quantum dilogarithm identity associated with this sequence. If the $c$ vector is $\left(\alpha_{1}, \alpha_{2}, \ldots, \alpha_{s}\right)$ and denote the sign of the $c$ vector as $\left(\epsilon_{1}, \epsilon_{2}, \ldots, \epsilon_{s}\right)^{12}$, then the quantum dilogarithm identity is

$$
\Psi_{q}\left(X^{\epsilon_{1} \alpha_{1}}\right)^{\epsilon_{1}} \cdots \Psi_{q}\left(X^{\epsilon_{s} \alpha_{s}}\right)^{\epsilon_{s}}=1
$$

The proof is the following [59]: since the cluster coordinates come back to itself up to permutation, we have the following identity

$$
A d\left(\Psi_{q}\left(X_{1}^{\epsilon_{1}}\right)^{\epsilon_{1}}\right) \tau_{1, \epsilon_{1}} \cdots A d\left(\Psi_{q}\left(X_{s}^{\epsilon_{s}}\right)^{\epsilon_{s}}\right) \tau_{s, \epsilon_{s}} \nu=1
$$

Now move all the $\tau_{i, \epsilon_{i}}$ to the far left, and we get

$$
A d\left(\Psi_{q}\left(X^{\epsilon_{1} \alpha_{1}}\right)^{\epsilon_{1}}\right) \cdots A d\left(\Psi_{q}\left(X^{\epsilon_{s} \alpha_{s}}\right)^{\epsilon_{s}}\right) \tau_{1, \epsilon_{1}} \cdots \tau_{s, \epsilon_{s}} \nu=1
$$

Using the relation $\tau_{1, \epsilon_{1}} \cdots \tau_{s, \epsilon_{s}} \nu=1$, we get the quantum dilogarithm identity in the desired form.

\footnotetext{
${ }^{12}$ Each $c$ vector is either all nonnegative or nonpositive, $\epsilon$ is the sign of the $c$.
} 
The relation to the wall crossing formula is the following: the sign of the $c$ vector are assembled into two groups, say $\alpha_{1}, \alpha_{2}, \ldots, \alpha_{r}$ has positive sign and the remaining ones has negative sign, we have

$$
\Psi_{q}\left(X^{\alpha_{1}}\right) \cdots \Psi_{q}\left(X^{\alpha_{r}}\right)=\Psi_{q}\left(X^{-\alpha_{r+1}}\right) \cdots \Psi_{q}\left(X^{-\alpha_{s}}\right),
$$

which gives the wall crossing formula for the $A_{2}$ quiver.

\section{BPS spectrum for $A_{1}$ theory}

Although the above mutation method is remarkably powerful, this approach has severe limitations without further knowledge of the quiver mutation structure. Without such knowledge, the above method is also kind of largely constrained to the ADE quiver class or its special generalization. The reason for the difficulty is due to the following two necessary conditions for doing green mutations:

1. A quiver with $2 n_{r}+n_{f}$ quiver nodes for a given $\mathcal{N}=2$ theory, here $n_{r}$ is the rank of the gauge group at the generic point of Coulomb branch and $n_{f}$ is the number of mass deformations.

2. A mutation sequence whose final quiver is isomorphic to the original one.

The first condition is by itself already highly non-trivial since usually there is no information about the BPS spectrum for most of theories. Our first claim is that the quivers and potential constructed in $[34,35]$ are the BPS quiver for the corresponding field theory. The second condition is even more difficult since the quiver for higher rank theory is of mutation infinite class, and it is very difficult to find a mutation sequence whose final quiver is isomorphic to the original one. Luckily, the construction given in $[34,35]$ also gives us the above very needed mutation sequence, which we will review in more detail in later sections.

In this section, we will mainly study the theory engineered using six dimensional $A_{1}$ theory which has been discussed in [18, 20, 21, 31, 60]. The study will be very useful for our later applications to the higher rank theory. Moreover, Using our method, it is pretty easy to recover the results in the literature, and in fact we can get a lot more finite BPS spectrums starting with arbitrary quivers, which is new. 


\subsection{Definition of the theory and BPS geometry}

In this section, we will consider four dimensional $\mathcal{N}=2$ theories derived by compactifying six dimensional $(2,0) A_{1}$ theory on a Riemann surface with regular singularity and irregular singularity. To study the BPS spectrum, each irregular singularity is replaced by a disc with marked points, and the number of marked points depend on the specific form of the irregular singularity, and the regular singularity is interpreted as puncture in the bulk. There is only one type of marked point since there is only one type of Young Tableaux of $A_{1}$ group. The BPS geometry is therefore a bordered Riemann surface with marked points on the boundary and punctures in the bulk.

5.1.1. Ideal triangulation. The BPS quiver is derived from the ideal triangulation of the corresponding bordered Riemann surface. Let us begin with a Riemann surface with boundaries and specify a finite set of points

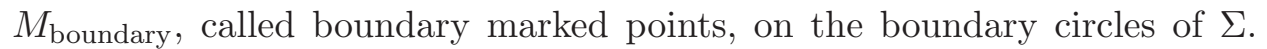
Each connected component of $\partial \Sigma$ has at least one boundary marked point; The bulk puncture is not blown up and remained as a point in the interior of the Riemann surface. The defining data of our theory is a triple $\left(\Sigma, M_{\text {boundary }}, p\right)$. For notational convenience we sometimes denote this triple simply by $\Sigma$. In other words, $\Sigma$ is defined by following data:

a. the genus $g$ of the Riemann surface;

b: the number of bulk punctures $p$.

d. the number $b$ of boundary components;

d. the number of marked points $h_{i}$ on each boundary.

Each puncture represents the regular singularity while the boundary with marked points means an irregular singularity, all the marked points have a Young Tableaux label. The punctures and the marked points are all called marked points for simplicity in the following, and one should be careful about whether it is in the bulk or one the boundary. One can define a combinatorial object called ideal triangulation on above Riemann surface. An ideal triangulation is defined using arcs [61]. A simple $\operatorname{arc} \gamma$ in $\Sigma$ is a curve such that

1. the endpoints of $\gamma$ are marked points;

2. $\gamma$ does not intersect itself, except at the endpoints;

3. $\gamma$ is disjoint from the marked points and the boundary.

We also require the arc $\gamma$ is not contractible into the marked points or onto the boundary. Each arc is considered up to isotopy. Two arcs are called compatible if they do not intersect in the interior of $\Sigma$. A maximal collection of distinct pairwise arcs is called an ideal triangulation. An edge 
is called external if it is isotopic to a segment of the boundary, otherwise it is called internal. It is not hard to get the following formula for the number of internal edges:

$$
6 g+3 b+3 p+\#\left|M_{\text {boundary }}\right|-6,
$$

where as defined previously $g(b)$ is the genus (the number of boundary components) of $\Sigma$, respectively. There are a total of $\#\left|M_{\text {boundary }}\right|$ external edges. Several examples for the ideal triangulations for various bordered Riemann surface are shown in Figure 16

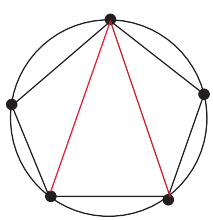

A

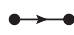

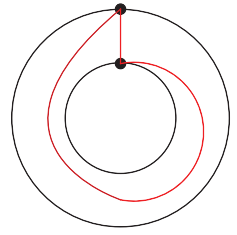

$B$

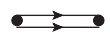

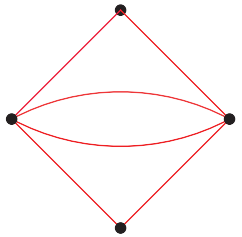

C

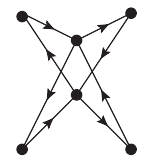

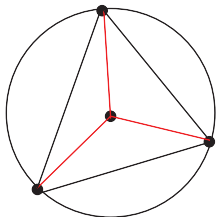

$D$

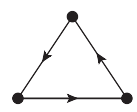

Figure 15: The triangulation of various BPS geometry. A: Disc with five punctures which represents $A_{2}$ Argyres-Douglas theory. B: Annulus with one marked point on each boundary, this is pure $S U(2)$ theory. C: Sphere with four punctures which is $S U(2)$ theory with four fundamental flavors. D: Disc with three marked points and a bulk puncture, which is the $D_{3}$ Argyres-Douglas theory.

We always start with an ideal triangulation without self-folded triangles, and the BPS quiver can be read from the triangulation in the following way:

1. Assign a quiver node to each internal edge of the triangulation.

2. There is a quiver arrow for two nodes if the two corresponding edges are in the same triangle. The total quiver arrows are the signed sum of the quiver arrows if the two nodes are in more than one triangles.

There are two special features for the quivers from the triangulated surface: first the maximal number of arrows are two for any two quiver nodes, second the quiver is in finite mutation class, namely the quiver will come back to itself after a finite number of mutations. 
The quantum field theory is formed by gauging two kinds of matter together ${ }^{13}$ : trifundamental of $S U(2)$ which is represented by the three punctured sphere and the $D$ type AD theory represented by a sphere with one irregular singularity and one regular singularity. Each bulk puncture contributes 3 to the charge lattice (one electric, one magnetic and one flavor charge), and each boundary with $n_{i}$ marked points contributes $\left(n_{i}+3\right)$ (when $n$ is even, there is a mass parameter), so the total dimension of the charge lattice is

$$
3 p+3 b+\#\left|M_{\text {boundary }}\right|-6+6 g
$$

which is equal to the number of the internal edges of the triangulation, so the BPS quiver from the triangulation has the right dimensions, and it can be checked that the rank of the quiver matrix is equal to $2 n_{r}$.

5.1.2. Potential. The potential for the quiver arising from the triangulation of the bordered Riemann surface is given in [62]. There is one potential term for the quiver arrows in each triangle and one term for each puncture. If there is a quadratic term in the potential, then the two quiver arrows are massive and can be integrated out and one get a reduced quiver and potential.

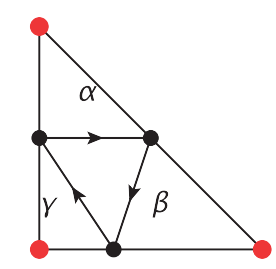

$W=\alpha \beta \gamma$

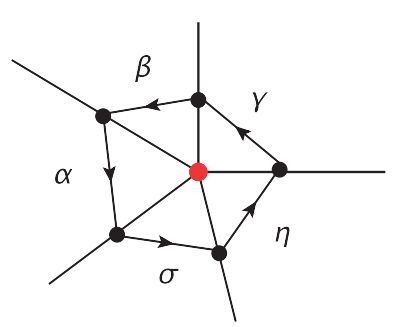

$W=\alpha \beta \gamma \eta \sigma$

Figure 16: There is a potential term for each triangle, and a potential term for each internal puncture.

Mathematically, the above integrating out process corresponds to removing two cycles in the quiver. The quiver defined in previous paragraph is actually the reduced one. In this paper, the quiver with potential $(Q, W)$ for a triangulation is always the reduced one.

\footnotetext{
${ }^{13}$ The one disc with marked points represents the $A_{n}$ type Argyres-Douglas theory.
} 
The triangulation of the BPS quiver is not unique and two different of triangulations are related by a sequence local moves called flips. It can be checked that the two quivers are related by mutations, and the reduced potential of two triangulations are also related exactly by the mutation rules, see Figure 17.

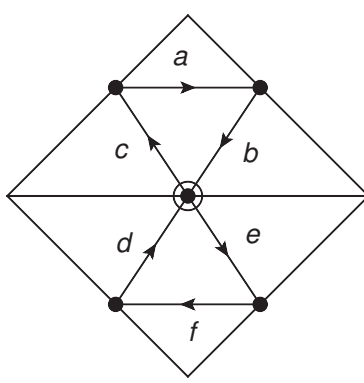

$W=a b c+d e f$

\section{flip}

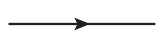

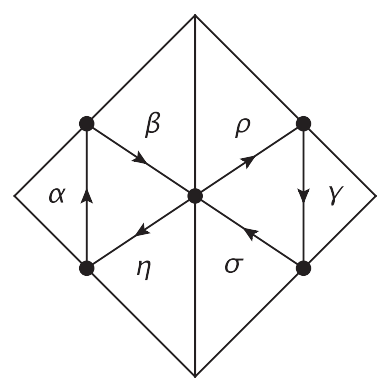

$W=\alpha \beta \eta+\rho \gamma \sigma$

Figure 17: The flip which relates two triangulations of the quadrilateral. The quivers are related by quiver mutation, and the potentials are also related by the mutation rule.

\subsection{Indecomposable objects: a geometric representation}

As we discussed earlier, the indecomposable representations of the quiver with potential are the possible BPS states. One could find them either from the indecomposable modules of the Jacobi algebra, or from the representation theory of $(Q, W)$. In this subsection, we are going to provide a geometric representation for these objects.

Let's consider the Riemann surface without the punctures, the Jacobi algebra defined from the triangulation is the so-called string algebra. As discovered in [63], the indecomposable modules of a string algebra are represented by the strings and bands. Let's fist give a definition of the strings and bands. Given an arrow $\beta$, and let $S(\beta)$ be its starting point and $e(\beta)$ its ending point. We denote $\beta^{-}$as the formal inverse of $\beta$ with $s\left(\beta^{-1}\right)=e(\beta)$ and $e\left(\beta^{-1}\right)=s(\beta)$, notice that $\left(\beta^{-}\right)^{-}=\beta$. A sequence of the quiver arrows (and their formal inverses) $\omega=\alpha_{1} \alpha_{2} \cdots \alpha_{n}$ is called a string if they satisfy the following two conditions

1. The ending point of $\alpha_{i}$ is the starting point of $\alpha_{i+1}$. 
2. The quiver arrows and its formal inverse are not appearing in sequel, i.e. $\alpha_{i} \neq \alpha_{i+1}^{-}$.

Thus a string $w$ for the Jacobi algebra is defined as a walk in the quiver avoid the zero relations from the potential:

$$
\omega: x_{1}-\alpha_{1} x_{2}-\cdots x_{n-1} \stackrel{\alpha_{n-1}}{-} x_{n}-\alpha_{n}-1
$$

Namely, there is no subsequence in $\omega$ which appears in the ideal $I$ defined by the potential. A string is called cyclic if $x_{1}=x_{n+1}$. A band is a simple cyclic string (the starting point and the ending point are the same for the string), i,e, it is not a power of any string. The dimensional vector of the string or band module is defined as

$$
d_{i}=\sum_{x \in \omega} \delta_{x, x_{i}}
$$

namely, the $i$ th component of the dimension vector is equal to the number of times the quiver nodes $x_{i}$ appears in the $\omega$.

The string and band module have a very nice geometric interpretation as the curves on the Riemann surface. The curve is required to be not homotopy to the curve of the triangulation and boundary component. The end points of open curve $\gamma$ without self-intersection are either on the puncture or on the boundary, such curves are representing the string module. A simple closed curve $l$ represents the band module. For two curves $\gamma$ and $\gamma^{\prime}$ in $\Sigma$, we denote by $I\left(\gamma, \gamma^{\prime}\right)$ as the minimal intersection number of two representatives of the homotopic classes of $\gamma^{\prime}$ and $\gamma$. Now the dimension vector of the module associated with a curve has the dimension vector associated with a triangulation

$$
d=\sum_{\gamma^{\prime} \in \Gamma} I\left(\gamma, \gamma^{\prime}\right)
$$

It is not hard to see that for the quiver from triangulated surface, the module from the open curve has no parameters and therefore represent the hypermultiplet, and the module from the closed curve has 1 parameter which then represents the $W$ boson. These curves then represent the possible BPS states, and our result is in perfect agreement with that found in [13]. The association of the vector multiplet from the closed curve is actually natural from the $M$ theory point of view, i.e the self-dual string wrapping on the closed curve gives the $W$ boson. 
Finally, let's give a simple application of the above example. The BPS geometry is a annulus with one marked points on each boundary, the triangulation and one closed curve is shown in Figure 18. The dimension vector for this curve is $(1,1)$ from the intersection number. The other string module has dimension $(n, n \pm 1)$ as seen from the open curves. These results match the result from the quiver representation theory.

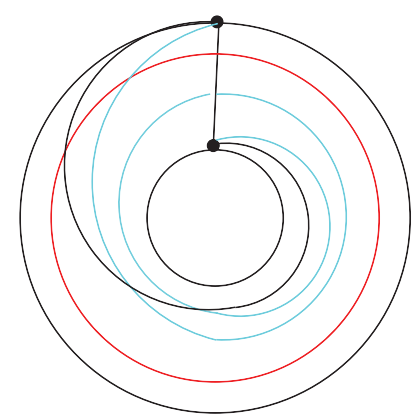

Figure 18: The red closed curve represents the vector boson, and the green curve represents the hypermultiplet.

The situation is more complicated for the Riemann surface with punctures, and the Jacobi algebra in that cases usually is not a string algebra. But we will make the following conjecture: the indecomposable modules associated with the $W$ boson is still represented by the closed curve, and one can read the subquiver from the intersection pattern. That is actually all we need for the later analysis.

\subsection{Finite cases: Disc and Disc with one puncture}

Let's consider a disc or a disc with one puncture in the bulk. When the BPS geometry is just a disc with $n+3$ marked points, it represents the $A_{n}$ Argyres-Douglas theory. The quiver from one of the ideal triangulation is of the $A_{n}$ shape which gives the name, see the left of Figure 19. The orientation of the quiver arrows is not important since they are all in the same quiver mutation class. These quivers have special property that there are finite number of indecomposable representations, and all of them represents the hypermultiplet. This fact means that all the chambers have finite number of hypermultiplets.

We are going to use the maximal green mutation to find the BPS spectrum of these theories by starting with the corresponding Dynkin diagram, 

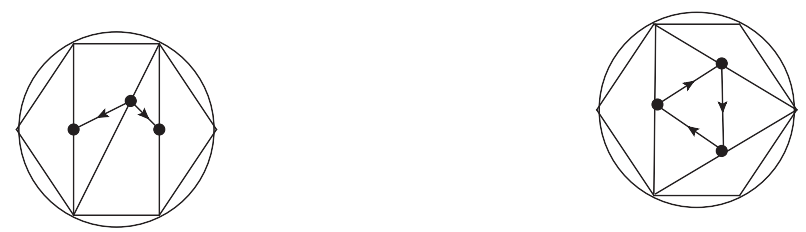

Figure 19: Two ideal triangulations of a disc with 6 marked points, which represents $A_{3}$ Argyres-Douglas theory. The BPS quiver is given too.

since there is only finite number of indecomposable object, one can do random green mutations and would definitely find a maximal green mutation at the end. The minimal chamber has $r$ BPS states, where $r$ is the number of simple roots of the corresponding lie algebra, and the number of BPS states in the maximal chamber is equal to the number of positive roots. The BPS states and charges are in one to one correspondence with the positive roots of lie algebra. The minimal chamber and maximal chamber is easy to find. Since the $A$ type quiver is acyclic, there is always a source node and sink node, the minimal chamber is found by always mutating the source node in each step, and the maximal chamber is found by mutating sink node only in each step, see Table 2. Moreover, for every integer $l$ satisfying $l_{\min } \leq l \leq l_{\max }$, there is a finite chamber with $l$ states. The interested reader can work out the charge vectors and the corresponding ordering of phase using the green mutations. The results of the minimal and maximal chamber of the ADE quiver are summarized in Table 1.

\begin{tabular}{|c|c|c|c|c|c|}
\hline & $A_{n}$ & $D_{n}$ & $E_{6}$ & $E_{7}$ & $E_{8}$ \\
\hline Minimal & $\mathrm{n}$ & $\mathrm{n}$ & 6 & 7 & 8 \\
\hline Maximal & $\frac{n(n+1)}{2}$ & $\mathrm{n}(\mathrm{n}-1)$ & 36 & 63 & 120 \\
\hline
\end{tabular}

Table 1: The number of BPS states in the minimal and maximal chamber for the ADE quiver.

Example 1. Let's consider the $A_{3}$ quiver with orientation $1 \rightarrow 2 \leftarrow 3$. We could easily list the chamber with three, four, five, and six BPS states, see Table 2. 


\begin{tabular}{|c|c|c|}
\hline & Maximal green mutation & charge vectors \\
\hline 3 & $\mu_{1}, \mu_{3}, \mu_{2}$ & $\gamma_{1}, \gamma_{3}, \gamma_{2}$ \\
\hline 4 & $\mu_{1}, \mu_{2}, \mu_{3}, \mu_{2}$ & $\gamma_{1}, \gamma_{2}, \gamma_{2}+\gamma_{3}, \gamma_{3}$ \\
\hline 5 & $\mu_{2}, \mu_{3}, \mu_{2}, \mu_{1}, \mu_{3}$ & $\gamma_{2}, \gamma_{2}+\gamma_{3}, \gamma_{3}, \gamma_{1}+\gamma_{2}, \gamma_{1}$ \\
\hline 6 & $\mu_{2}, \mu_{3}, \mu_{1}, \mu_{2}, \mu_{3}, \mu_{1}$ & $\gamma_{2}, \gamma_{2}+\gamma_{3}, \gamma_{1}+\gamma_{2}, \gamma_{1}+\gamma_{2}+\gamma_{3}, \gamma_{1}, \gamma_{3}$ \\
\hline
\end{tabular}

Table 2: The maximal green mutation sequences and charge vectors for $A_{3}$ quiver.

If we start with a quiver which is mutation equivalent to the $A_{n}$ quiver, one can do random green mutations too. This fact is rather interesting since the maximal green mutations actually knows about the potential, although we did not specify the potential in the definition of green mutations. In fact, the green mutation is desired for the generic potential of the quiver, namely, one can mutate the quiver and always get a 2 -acyclic quiver due to the potential.

The new issue is that the number of BPS states in the minimal chamber and the maximal chamber do not necessarily equal to the number given by the Dynkin diagram. For example, consider the quiver in Figure 20, the minimal chamber has 4 BPS states instead of 3. The mutation sequence corresponding to the minimal chamber is

$$
\mu_{1}, \mu_{2}, \mu_{3}, \mu_{1} .
$$

The maximal chamber of this quiver has 5 states. So the number of states in minimal chamber and maximal chamber is not an quiver invariant. This is natural from the green mutation subset point of view: the quiver subset of the minimal and maximal chamber of $A_{n}$ quiver is always acyclic and so it would not include the above cyclic quiver, so the minimal chamber or maximal chamber is not realized in the cyclic quiver.

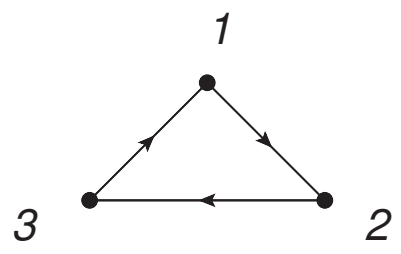

Figure 20: Quiver which is mutation equivalent to the $A_{3}$ quiver, and there is a potential term for the cyclic path in the quiver. 
The BPS geometry of $D$ type AD theory is realized as a disc with one puncture on the bulk. The BPS quiver can be found easily from the triangulation and it is indeed of the $D$ shape in one triangulation, see Figure 21. This is of the finite type and one could easily find the finite chamber and the charge vectors by doing random green mutations.
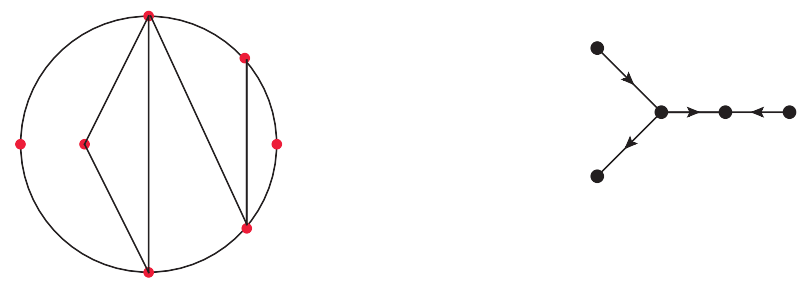

Figure 21: The triangulation of the disc with one puncture in the bulk. The quiver is of the $\mathrm{D}$ shape.

The $E$ type AD theory can be found using $A_{2}$ theory compactified on a sphere with one specific irregular singularity as shown in [25]. The BPS spectrum can be similarly found using random green mutations, and the minimal and maximal chamber is listed in Table 1.

\subsection{Riemann surface without punctures}

In this subsection, we are going to study theory whose BPS geometry is a bordered Riemann surface without bulk punctures, and the Jacobi algebra of this class of theories are string algebra, and in particular the vector boson and its corresponding quiver representation can be easily found from the closed curves on the BPS geometry. By inspection, the subquiver for the vector boson is always acyclic and there are definitely a source node and a sink node. Let's denote the charge vectors of the source node as $\gamma_{\text {source }}$ and the sink node as $\gamma_{\text {sink }}$. We now argue that the sufficient condition for the $W$ boson to be unstable is that the source node has larger slop than the the sink node.

$$
\mu\left(\gamma_{\text {source }}\right)>\mu\left(\gamma_{\text {sink }}\right)
$$

Let's prove the above statement using the quiver representation theory. The $W$ boson representation $P$ has the following two special subrepresentations

$$
P_{1}=\gamma_{\text {sink }}, \quad P_{2}=\sum_{i-\text { source }} \gamma_{i}
$$


The condition for $P$ to be stable is that the slop of $P$ should be smaller than all of its subrepresentation, namely $P_{1}$ and $P_{2}$ should be on the left of $P$ on the half plane:

$$
\mu(P)<\mu\left(P_{1}\right) \quad \& \quad \mu(P)>\mu\left(P_{2}\right)
$$

If $P$ is stable, $\operatorname{since} \operatorname{dim}(P)=\operatorname{dim}\left(P_{2}\right)+\operatorname{dim}\left(P_{\text {source }}\right)$ and $P_{2}$ is on the left of $P$, then $P_{\text {source }}$ is definitely on the right of $P$, this implies that

$$
\mu\left(P_{\text {source }}\right)<\mu(P)<\mu\left(P_{\text {sink }}\right)
$$

In another word, as long as the slop of the source node is larger than the sink node, the $W$ boson is definitely unstable. Notice that this is not the necessary condition to find the finite spectrum. In practice, this means that if the source charge appears before the sink charge in the green mutation sequence, then this $W$ boson would be killed.

For simple BPS geometry, one can find all possible finite chambers using the random mutations without worrying about the $W$ boson. Some simple BPS geometry of this sort and the minimal plus maximal chamber are listed in Table 3 . There is a finite chamber for all the integers $l_{\min } \leq l \leq l_{\max }$. The minimal chamber is easy to find: there is a source node in the quiver, and one mutate the source node in each step.

\begin{tabular}{|c|c|c|c|}
\hline Quiver & theory & minimal & maximal \\
\hline$\tilde{A}(1,1)$ & $S U(2)$ & 2 & 2 \\
\hline$\tilde{A}(2,1)$ & $S U(2)$ with $N_{f}=1$ & 3 & 5 \\
\hline$\tilde{A}(2,2)$ & $S U(2)$ with $N_{f}=2$ & 4 & 10 \\
\hline$\tilde{D}_{4}$ & $S U(2)$ with $N_{f}=3$ & 5 & 22 \\
\hline Figure $26 \mathrm{~A}$ & $S U(2)$ with $N_{f}=4$ & 12 & 46 \\
\hline
\end{tabular}

Table 3: The minimal and maximal chamber for $S U(2)$ with $N_{f} \leq 4$.

For more complicated geometry, one can either find the finite chamber using the random green mutations, or use the following steps:

a. Identify the subquiver corresponding to the $W$ boson which is represented by the closed curve in the Riemann surface, and identify the source node and sink node of this subquiver.

b. Do the green mutation such that the charge of the source node appear before the charge of the sink node. The easiest thing you can do is to mutate source node first. 
Let's consider an annulus with $n_{1}$ marked points and $n_{2}$ marked points on each boundary. The BPS quiver from one specific triangulation has the form of affine $\tilde{A}\left(n_{1}, n_{2}\right)$ shape where there are $n_{1}$ arrows in one direction, and $n_{2}$ arrows in another direction, see Figure 22. There is only one closed curve and so only one $W$ boson whose corresponding quiver representation $P$ has dimension vector $(1,1, \ldots, 1)$. Since node 1 is the source node of this subquiver, $W$ boson is definitely unstable if node 1 is mutated first, and we will always find a finite chamber by doing random green mutation afterwards.
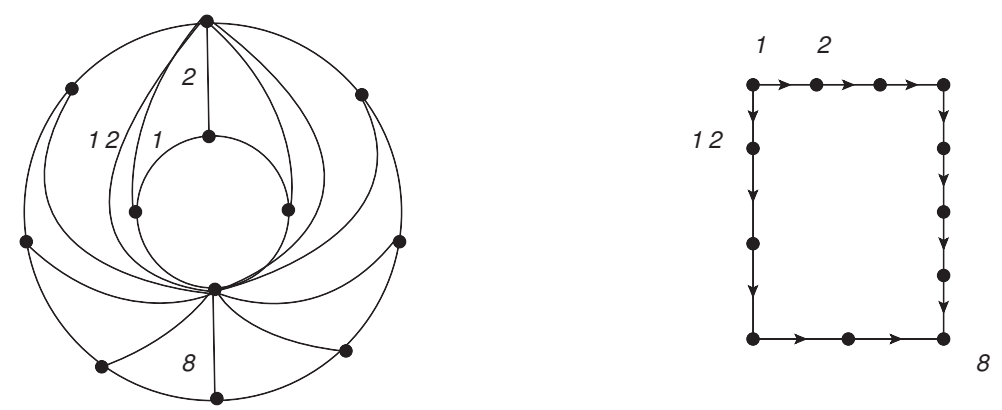

Figure 22: The triangulation of an annulus with 4 points on one boundary and 8 points on another boundary; The quiver is shown on the left which is of the affine Dynkin diagram $\tilde{A}(4,8)$.

Example 2. Here is another theory whose BPS geometry is a sphere with three boundary each with a marked point, and the four dimensional theory is three $S U(2)$ gauge groups coupled through a trifundamental, so the BPS quiver should have six quiver nodes. The triangulation and quiver are shown in Figure 23, and there are three closed curves which represent three $W$ bosons. The sink-source analysis of these bands are listed in Table 4. So if we mutate node 1 and node 3 first, then the three $W$ bosons would be unstable, and random green mutations can be done later. A maximal green mutation sequence is

$$
\mu_{1}, \mu_{3}, \mu_{2}, \mu_{4}, \mu_{6}, \mu_{5}, \mu_{3}, \mu_{4}
$$

\subsection{Riemann surface with punctures}

5.5.1. Asymptotical free theory. The BPS geometry for $S U(2)$ with $N_{f}=3$ requires the bulk puncture: it is a disc with two marked points 

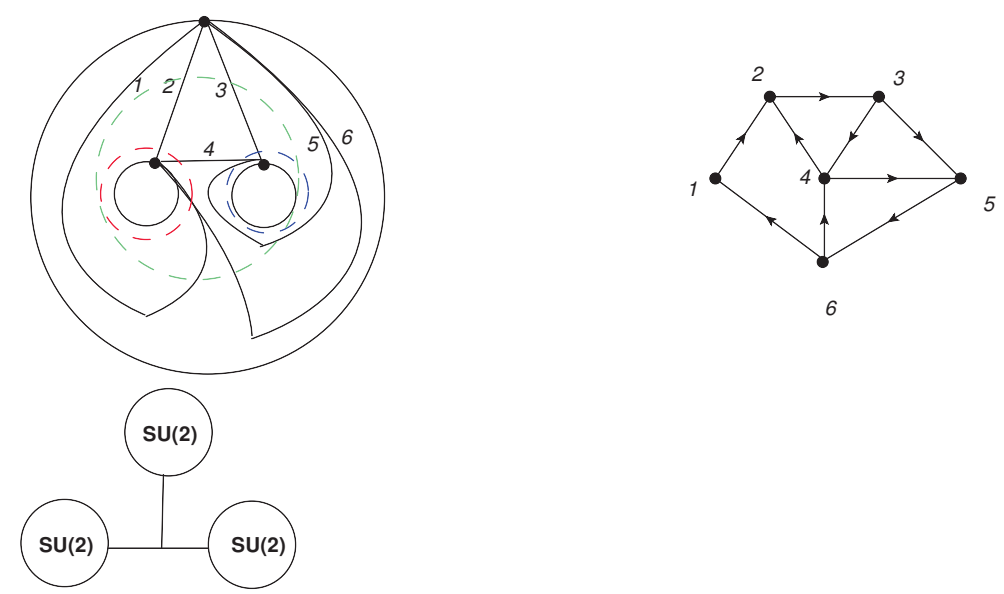

Figure 23: The triangulation of a sphere with three boundaries each with one marked point, and the quiver gauge theory underlying this BPS geometry is shown too. The three closed curves representing the $W$ bosons are drawn from which one can read the subquiver for them. The BPS quiver is shown on the right.

\begin{tabular}{|c|c|c|c|}
\hline Band & quiver nodes & source & sink \\
\hline$B_{1}$ & $(1,2,4,6)$ & 1 & 2 \\
\hline$B_{2}$ & $(1,2,3,5,6)$ & 1 & 6 \\
\hline$B_{3}$ & $(3,4,5)$ & 3 & 5 \\
\hline
\end{tabular}

Table 4: The source and sink nodes for the three bands from the quiver in Figure (23).

and two bulk punctures. One triangulation and the BPS quiver is shown in Figure 24. This quiver has the affine $\tilde{D}_{4}$ shape, and more generally the quiver is $\tilde{D}_{n+2}$ in one of the triangulation if there is two bulk punctures and $n$ marked point on boundary of the disc. There is one closed curve representing the $W$ boson for the gauge group, and its dimension vector is $(1,1,2,1,1)$. Since the affine $\tilde{D}_{4}$ quiver is acyclic, the number of BPS states in minimal chamber has 5 states and can be found using the source sequences. The maximal chamber has 22 states as found by doing all possible green mutations. 

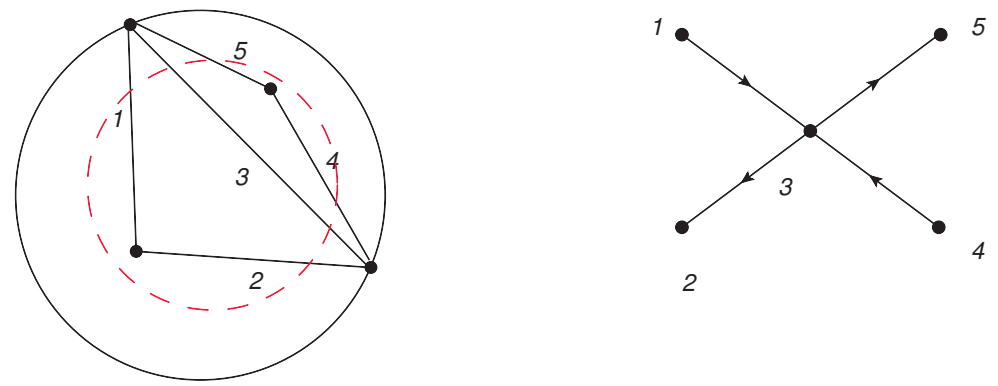

Figure 24: An ideal triangulation of twice punctured disc and the corresponding quiver.

Let's now give a conjecture on the number of states in the maximal chamber from the the following assumption: the number of states is a continuous function of the parameters of the BPS geometry. Consider affine $\tilde{D}_{n}$ quiver, we further conjecture that it is of the order of $n^{2}$ :

$$
f(n ; 2)=a n^{2}+b n+c,
$$

when $n=0$, the geometry becomes a three puncture sphere, but only the mass deformations corresponding to bulk punctures are allowed, therefore there are only two BPS states. When $n=1$, the underlying theory is a $S U(2)$ with two flavors, so $f(1)=10$, finally $f(2)=22$ as from the experimental study. Using these initial data, we conjecture the maximal chamber has the following number of states:

$$
f(n ; 2)=2 n^{2}+6 n+2 .
$$

which is in agreement with the result found from computer scanning in [64].

The above analysis can actually be generalized to other type of BPS geometry. Let's an annulus with 1 and $n$ marked points on each boundary, and the BPS quiver in one particular triangulation is the affine $\tilde{A}(n, 1)$ quiver. The minimal chamber has $n+1$ states, let's use $f(n, 1)$ to represent the number of states in maximal chamber and again assume the number of states is a quadratic function of $n$. Using the initial data $f(0,1)=0$ (since this is a trivial theory), $f(1,1)=2$ for pure $S U(2)$ theory, and $f(2,1)=5$ for $S U(2)$ with one flavor, we find

$$
f(n, 1)=\frac{n^{2}+3 n}{2}
$$




\begin{tabular}{|c|c|c|c|c|}
\hline BPS geometry & Quiver & theory & minimal & maximal \\
\hline Annulus: $B_{1}, B_{n}$ & $\tilde{A}(n, 1)$ & $S U(2)-A D$ & $\mathrm{n}+1$ & $\frac{n(n+3)}{2}$ \\
\hline$B_{n}$, two punctures & $\tilde{D}_{n+2}$ & $2-S U(2)-A D$ & $n+3$ & $2 n^{2}+6 n+2$ \\
\hline
\end{tabular}

Table 5: The minimal chamber and maximal chamber for two class of BPS geometry.

More generally, consider an annulus with $n_{1}$ and $n_{2}$ marked points on each boundary, and the BPS quiver is the affine quiver $\tilde{A}\left(n_{1}, n_{2}\right)$ from one particular triangulation. The number of states in maximal chamber should be invariant under exchange of $n_{1}$ and $n_{2}$, so

$$
f\left(n_{1}, n_{2}\right)=f\left(n_{1}+n_{2}, n_{1} n_{2}\right)
$$

and it should be a quadratic polynomial from our assumption. Denote $x=$ $n_{1}+n_{2}, y=n_{1} n_{2}$, then the general expression reads

$$
f\left(n_{1}, n_{2}\right)=a x^{2}+b y^{2}+c x y+d x+e y+f .
$$

Using the result for $f(n, 1), f(2,2)=10, f(3,0)=5$, and $f(4,0)=9,{ }^{14}$ the number of states in maximal chamber is

$$
f\left(n_{1}, n_{2}\right)=\frac{1}{2} x^{2}+\frac{1}{4} y^{2}-\frac{1}{4} x y+\frac{1}{2} x+\frac{1}{4} y-1 .
$$

Immediately, we make the prediction that the maximal chamber of the quiver $\tilde{A}(n, 0)$ has states

$$
f(n, 0)=1 / 2\left(-2+n+n^{2}\right) .
$$

It would be interesting to prove our this conjecture using computer scanning, etc.

Now let's give an example showing how to use the generalized sourcesink sequence to find the finite chamber for the theory defined using the bulk puncture.

Example 3. Consider a Riemann surface with two boundaries with a single marked point and a bulk puncture. The $\mathcal{N}=2$ theory is $S U(2) \times S U(2)$

\footnotetext{
${ }^{14}$ Notice that disc with one bulk puncture is representing D type Argyres-Douglas theory, but the quiver is of the cyclic affine type, so the maximal number of states is different from the one derived from the $\mathrm{D}$ type Dynkin diagram as listed in Table 1.
} 
asymptotical quiver gauge theory. One triangulation and the quiver are shown in Figure 25.
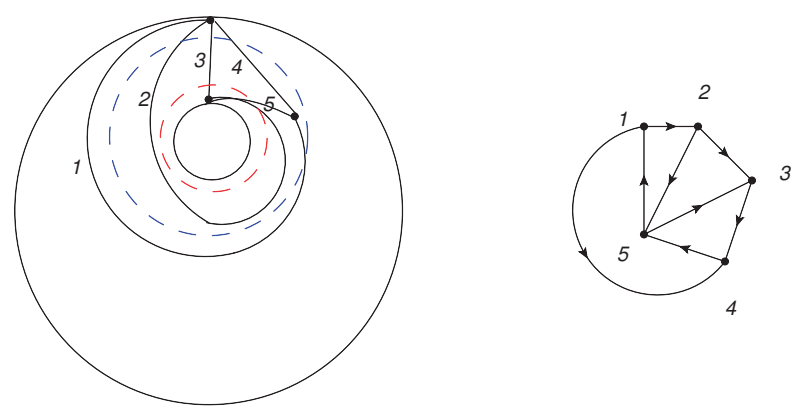

Figure 25: The quiver of two boundaries with a single marked point plus a bulk puncture, the $W$ boson is represented by closed curve.

There are two closed curves representing $W$ bosons for two $S U(2)$ gauge groups. The subquvier corresponding to these two bands and the sourcesink nodes are shown in Table 6 . Since 2 and 1 are the source nodes for

\begin{tabular}{|c|c|c|c|}
\hline Band & quiver nodes & source & sink \\
\hline$B_{1}$ & $(2,3,5)$ & 2 & 3 \\
\hline$B_{2}$ & $(1,2,3,4)$ & 1 & 4 \\
\hline
\end{tabular}

Table 6: The source and sink nodes for the two bands of the quiver shown in Figure (25).

two bands, and if we mutate node 1 and node 2 first, then the $W$ bosons will be disabled and a finite chamber can be found by doing random green mutations. We just list one chamber below

$$
\mu_{1}, \mu_{2}, \mu_{5}, \mu_{1}, \mu_{3}, \mu_{4}, \mu_{1}
$$

There are many other possibilities which could be easily found from the green mutations.

\subsubsection{Fourth punctured sphere.}

Example 4. Consider the fourth punctured sphere which represents $S U(2)$ theory with four flavors. The BPS quiver from one triangulation is shown in Figure 26A. Now there are three closed curves and we list the source and sink nodes in Table 7. 


\begin{tabular}{|c|c|c|c|}
\hline Band & quiver nodes & source & sink \\
\hline$B_{a, b}$ & $(2,5,4,6)$ & 5 & 6 \\
\hline$B_{a, c}$ & $(1,5,3,6)$ & 6 & 5 \\
\hline$B_{a, d}$ & $(5,1,2,3,4)$ & $(1,3)$ & $(2,4)$ \\
\hline
\end{tabular}

Table 7: Source and sink nodes for three bands of the quiver of $S U(2)$ with four flavors, see Figure (26)A.

We want charge vectors $\gamma_{5}, \gamma_{6}, \gamma_{1}$ to appear first in doing green mutations ${ }^{15}$, which can be done by doing the mutation sequences $\mu_{6}, \mu_{1}, \mu_{5}$, which will ensure all the $W$ bosons are unstable. Then we can do green mutation in a random way. One sequence is the following

$$
\begin{aligned}
& \mu_{6}, \mu_{1}, \mu_{5}, \mu_{2}, \mu_{3}, \mu_{4}, \mu_{3}, \mu_{1}, \\
& \mu_{4}, \mu_{6}, \mu_{5}, \mu_{3}, \mu_{5}, \mu_{4}, \mu_{2}, \mu_{3}, \mu_{5} .
\end{aligned}
$$

and this chamber has 17 states. There are many other possibilities for the maximal green mutations, and this example has been studied extensively in [64]. The result is that the maximal chamber has 46 states and the minimal chamber has 12 states. The minimal chamber can be found using the following mutation sequences

$$
\left(\mu_{5}, \mu_{6}\right),\left(\mu_{1}, \mu_{2}, \mu_{3}, \mu_{4}\right),\left(\mu_{5}, \mu_{6}\right),\left(\mu_{1}, \mu_{2}, \mu_{3}, \mu_{4}\right) .
$$

If we start with another triangulation as shown in Figure 26B, then there are also three subquivers for $W$ bosons and the source, sink nodes are shown in Table 8. We found the following special beginning sequences which will

\begin{tabular}{|c|c|c|c|}
\hline Band & quiver nodes & source & sink \\
\hline$B_{a, d}$ & $(4,6,2,5)$ & $(5,6)$ & $(2,4)$ \\
\hline$B_{a, b}$ & $(3,6,1,5)$ & $(1,3)$ & $(6,5)$ \\
\hline$B_{a, c}$ & $(1,2,4,3)$ & $(2,4)$ & $(1,3)$ \\
\hline
\end{tabular}

Table 8: The source and sink analysis for the bands of another quiver from $S U(2)$ with four flavors.

\footnotetext{
${ }^{15}$ Equivalently, we can do the green mutation such that charge $\gamma_{5}, \gamma_{6}$ and $\gamma_{3}$ appear first.
} 

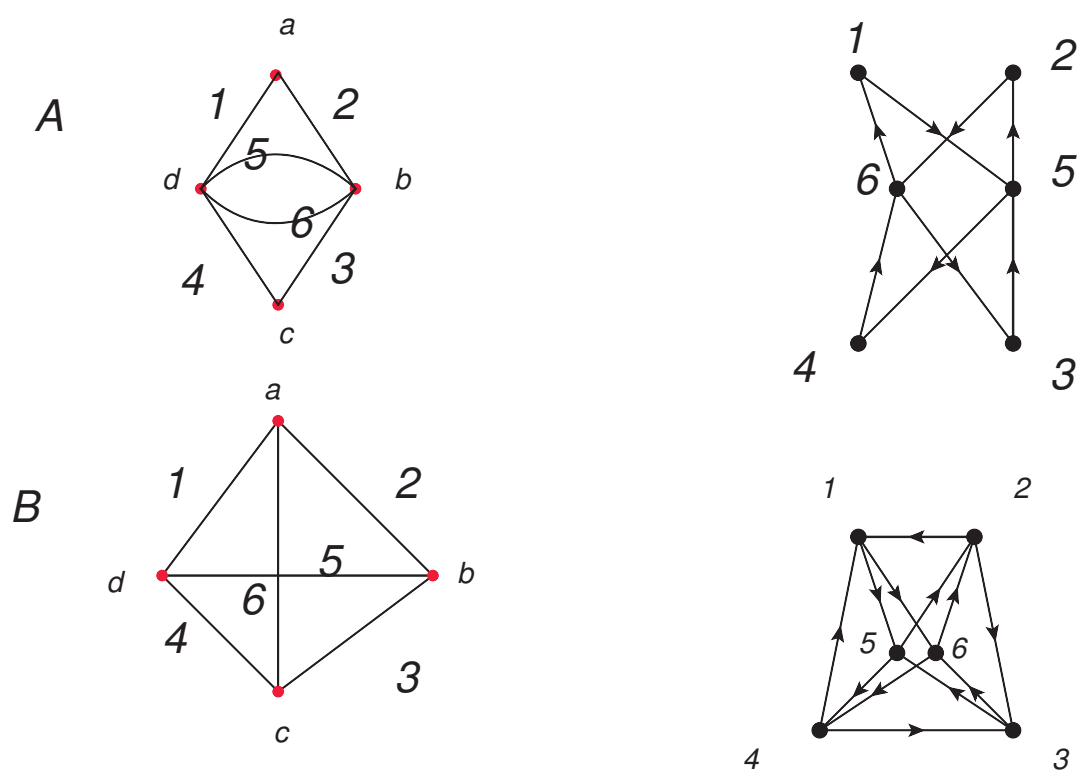

Figure 26: A: one triangulation of fourth punctured sphere and the quiver. B: another triangulation of fourth punctured sphere and the quiver.

make $W$ boson unstable:

$$
\mu_{5},\left(\mu_{2}, \mu_{1}\right), \mu_{5},
$$

because this sequence will produce the following charge vectors,

$$
\gamma_{5}, \gamma_{2}, \gamma_{1}+\gamma_{5}, \gamma_{1}
$$

which will ensure the source charges of each band appear before that of the sink node. After these steps, one can do random green mutations, i.e. the following mutation sequences are the maximal one:

$$
\mu_{5}, \mu_{2}, \mu_{1}, \mu_{5}, \mu_{6}, \mu_{3}, \mu_{6}, \mu_{4}, \mu_{6}, \mu_{2}, \mu_{1}, \mu_{2}, \mu_{5}, \mu_{2} \text {. }
$$

The evidence for $S$ duality. Notice there are three closed curves and therefore three possible $W$ boson, which indicate that there are three duality frames and each $W$ boson represents a duality frame. However, a natural question for the consistency is whether they can appear in a single chamber. We will prove that this can not happen by studying the quiver representation of quiver in Figure 26A. Let's first list all the sub representations of the three bands, see Table 9 . 
First of all, band $B_{a, b}$ and $B_{a, c}$ can not be coexisting since the necessary condition for the stability of the bands is that the slop of the source node is smaller than the sink node, this is can not be satisfied simultaneously for two bands since node 5 and node 6 exchange the role of source and sink in two bands. Next consider the pair of bands $B_{a, d}$ and $B_{a, b}$, they share a comment subquiver $2 \leftarrow 5 \rightarrow 4$, now to make $B_{a, d}$ stable, we need to mutate this subquiver first such that all the charge vectors $\gamma_{2}, \gamma_{4}, \gamma_{5}$ appear, but his automatically will make $B_{a b}$ unstable since $\mu\left(\gamma_{5}\right)>\mu\left(\gamma_{6}\right)$ (the slop of the source charge is bigger than the sink charge)! Similar analysis applies to the bands $B_{a, d}$ and $B_{a, c}$.

\begin{tabular}{|c|c|c|}
\hline Band & quiver nodes & Subrepresentation \\
\hline$B_{a, b}$ & $(2,5,4,6)$ & $\gamma_{2}+\gamma_{4}+\gamma_{6}, \gamma_{2}+\gamma_{6}, \gamma_{4}+\gamma_{6}, \gamma_{6}$ \\
\hline$B_{a, c}$ & $(1,5,3,6)$ & $\gamma_{1}+\gamma_{3}+\gamma_{5}, \gamma_{1}+\gamma_{5}, \gamma_{3}+\gamma_{5}, \gamma_{5}$ \\
\hline$B_{a, d}$ & $(5,1,2,3,4)$ & $\gamma_{2}+\gamma_{3}+\gamma_{4}+\gamma_{5}, \gamma_{2}+\gamma_{5}+\gamma_{4}, \gamma_{2}, \gamma_{4}, \gamma_{1}+\gamma_{2}+\gamma_{5}+\gamma_{4}$ \\
\hline
\end{tabular}

Table 9: Subrepresentations of various bands of $S U(2)$ with four fundamentals.

It is possible to generalize the above consideration to other theories defined from the bordered Riemann surface. Our conjecture is that there are only a maximal set of non-intersected closed curves whose $W$ boson can become simultaneously stable.

\subsubsection{Sphere with five punctures.}

Example 5. Let's consider a sphere with five punctures, which represents the conformal quiver gauge theory $2-S U(2)-S U(2)-2$. A triangulation and the corresponding quiver are shown in Figure 27. The subquiver and source-sink analysis of various bands are listed in the following Table 10.

Now let's take two disjoint triangles, say $\Delta_{123}$ and $\Delta_{789}$, and do the mutation sequences

$$
\begin{aligned}
& \text { Step } 1: \mu_{1},\left(\mu_{2}, \mu_{3}\right), \mu_{1}, \\
& \text { Step } 2: \mu_{7},\left(\mu_{8}, \mu_{9}\right), \mu_{7} .
\end{aligned}
$$

This mutation will create the charge vectors

$$
\gamma_{1}, \gamma_{1}+\gamma_{2}, \gamma_{2}, \gamma_{3}, \gamma_{7}, \gamma_{7}+\gamma_{8}, \gamma_{8}, \gamma_{9}
$$



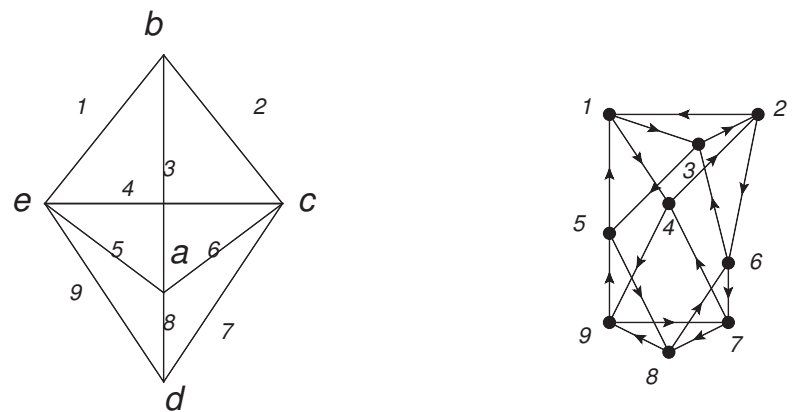

Figure 27: A triangulation and quiver of the sphere with five punctures.

\begin{tabular}{|c|c|c|c|}
\hline Band & quiver nodes & source & sink \\
\hline$B_{a, b}$ & $(1,5,8,6,2)$ & $(2,5)$ & $(1,6)$ \\
\hline$B_{a, c}$ & $(7,8,5,3,2,4)$ & $(3,7)$ & $(8,2)$ \\
\hline$B_{a, d}$ & $(5,3,6,7,9)$ & $(6,9)$ & $(7,5)$ \\
\hline$B_{a, e}$ & $(9,8,6,3,1,4)$ & $(8,1)$ & $(3,9)$ \\
\hline$B_{b, c}$ & $(7,6,3,1,4)$ & $(6,1)$ & $(3,4)$ \\
\hline$B_{b, e}$ & $(9,5,3,2,4)$ & $(3,4)$ & $(2,5)$ \\
\hline$B_{c, d}$ & $(2,4,9,8,6)$ & $(4,8)$ & $(6,9)$ \\
\hline$B_{c, e}$ & $(7,6,2,1,5,9)$ & $(2,9)$ & $(1,7)$ \\
\hline$B_{d, e}$ & $(1,4,7,8,5)$ & $(5,7)$ & $(4,8)$ \\
\hline
\end{tabular}

Table 10: The source and sink analysis of the bands of the sphere with five punctures.

and they will kill all the $W$ boson! For example, for the band $B_{a b}$, the source charge vector $\gamma_{2}$ appears before the sink $\gamma_{6}$, so it is unstable. After the above step, we will always find the finite chamber by doing random mutation sequences. A maximal green mutation sequences are

$$
\begin{aligned}
& \mu_{1},\left(\mu_{2}, \mu_{3}\right), \mu_{1}, \mu_{7},\left(\mu_{8}, \mu_{9}\right), \mu_{7}, \mu_{6}, \mu_{3}, \mu_{8}, \mu_{5}, \mu_{8}, \\
& \mu_{2}, \mu_{6}, \mu_{7}, \mu_{4}, \mu_{3}, \mu_{6}, \mu_{7}, \mu_{4}, \mu_{3}, \mu_{1}, \mu_{8}, \mu_{9}, \mu_{4}, \mu_{3} .
\end{aligned}
$$

One can do similar analysis for sphere with more punctures, although the analysis would become very tedious. In next section, we are going to describe another simpler method to find the finite chamber using the higher rank realization of the same theory. 


\section{BPS spectrum for $A_{N-1}$ theory}

A large class of four dimensional $\mathcal{N}=2$ field theory can be engineered by compactifying six dimensional $A_{N-1}(2,0)$ theory on a Riemann surface with regular singularity and irregular singularity ${ }^{16}$. The geometric data defining the theory is:

1. A Riemann surface $M_{g, p_{i}, b_{j}}$, where $g$ is the genus, $p_{i}$ is the regular singularity, and $b_{j}$ is the irregular singularity.

2. $p_{i}$ is classified by the Young Tableaux ${ }^{17}[24,65]$, and $b_{j}$ is classified by a Newton Polygon [25] ${ }^{18}$.

Let's describe a little bit about the the four dimensional theory defined by various geometries. The Riemann surface $M_{g, p_{i}, 0}$ defines a four dimensional superconformal field theory whose gauge coupling constants are identified by the complex structure moduli of $M$. Different duality frames are realized as different degeneration limits of the same Riemann surface. Weakly coupled gauge theory description in each duality frame is completely determined by the genus and the Young Tableaux type, and generically the theory is formed by gauging the flavor symmetries of the strongly coupled isolated SCFT defined by the three punctured sphere. Many properties of these theories including S duality [24, 66], Seiberg-Witten curve, 3d mirrors [30], central charges, and superconformal index [29] can be understood from this beautiful geometric construction.

The Riemann surface $M_{0, p, b}$ (one regular and one irregular singularity on the sphere) and $M_{0,0, b}$ (only one irregular singularity on the sphere) define another type of SCFT called Argyres-Douglas theory, which is typically an isolated theory (without marginal deformations) and has fractional scaling dimension for the operator spectrum. Lots of properties regarding these type of theories are studied in detail in [35].

In general, $M_{g, p_{i}, b_{j}}$ defines a four dimensional $\mathcal{N}=2$ theory which in each duality frame is formed by gauging the flavor symmetries of the following two types of matters: AD type theory represented by a sphere with one irregular singularity and one regular singularity, and the isolated SCFT represented by a sphere with three regular singularities.

The BPS geometry of the underlying field theory can be derived by blowing up the irregular singularity, and each irregular singularity is replaced by

\footnotetext{
${ }^{16}$ Roughly speaking, regular singularity means first order pole while the irregular singularity means the higher order pole.

${ }^{17} \mathrm{We}$ call a puncture full if the Young Tableaux has the form $[1,1, \ldots, 1]$, and simple if the Young Tableaux is $[N-1,1]$.

${ }^{18}$ The degenerating case needs further data, i.e. a sequence of Young Tableaux.
} 
a boundary with marked points labeled also by Young Tableaux. The detailed map between the irregular singularity and the corresponding marked boundary is worked out in [35]. The bordered Riemann surface relevant for the construction of ideal triangulation is depicted in the Figure 28. Once an ideal triangulation is given, the BPS quiver can be found from this geometric data by introducing more structures into each triangle of the ideal triangulations. In next subsection, we will describe the construction of the BPS quiver in more detail.
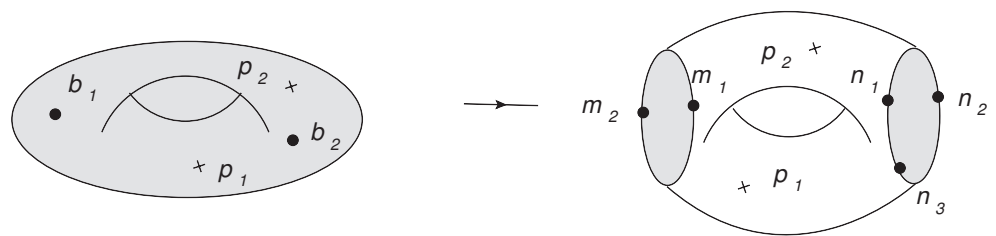

Figure 28: Left: The Riemann surface with regular and irregular singularity defines a four dimensional UV complete $\mathcal{N}=2$ theory; Right: The BPS geometry for the corresponding $4 \mathrm{~d}$ theory is derived by replacing each irregular singularity with a boundary with marked points labeled by Young Tableaux.

\subsection{Dot diagram, network and quiver}

Let's review the results presented in [34] which discussed how to find the BPS quiver for the higher rank $\mathcal{N}=2$ theory defined by $M_{g, p_{i}, b_{j}}$. The construction starts with an ideal triangulation of the bordered Riemann surface and a choice of cyclic path connecting all the punctures in the triangulation. The difference from the $A_{1}$ case is that more structures are needed to put on the edges and inside each triangle: there are more than one quiver node on each edge and there are quiver nodes inside each triangle.

The basic ingredient is attach a quiver to a single triangle with different Young Tableaux at the vertex, and the full quiver is derived by gluing the triangle quivers together. Given a triangle labeled by three Young Tableaux $\left(Y_{1}, Y_{2}, Y_{3}\right)$ in a cyclic order, one could find a dot diagram and a tessellation of the triangle using the brane construction proposed in [67]. Let's put the triangle inside a two dimensional lattice with unit spacing, and put the three vertices at positions $(N, 0),(0,0)$, and $(0, N)$. The dot diagram for the lattice points bounded by the triangle (including the points on the boundary) is found as follows: 
a. Decorating the boundary edge of the triangle with black dots and white dots using $Y$ vertex right ahead of it in the clockwise direction: If the Young Tableaux $Y$ has partition $\left[n_{1}, n_{2}, \ldots, n_{s}\right]$, then first put $n_{1}-1$ white dots and a black dots to represent the $n_{1}$ column, and the second step is done by putting $n_{2}-1$ white dots and 1 black dot, and continue this way until the whole Young Tableaux is represented by the black-white pattern on this boundary edge.

b. Constructing the dot diagram inside the triangle using only following two types of polygons whose edge is formed by lines ${ }^{19}$ connecting two black dots. 1: Triangles whose edges have the same lengths. 2: Trapeziums whose parallel sides have lengths $n_{1}, n_{2}$ and the other two sides have length $n_{1}-$ $n_{2}{ }^{20}$.

There are two types of polygons in the dot diagram: The type A polygon is the one whose triangle completion has the same orientation as the big triangle, and the type B polygon has opposite orientation. A bipartite 21 network and the quiver can be constructed directly from the dot diagram ${ }^{22}$ : we put a colored vertex inside each polygon using the following rule (see Figure 29):

a: Assign a white vertex to each type A polygon.

b: Assign a black vertex to each type B polygon.
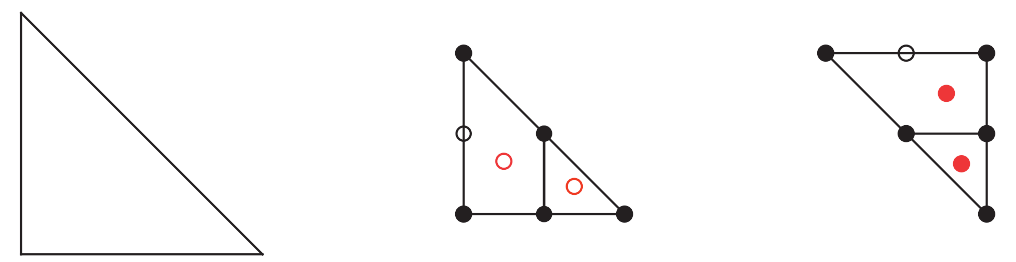

Figure 29: Left: The orientation of the big triangle. Middle: Put a white vertex to each polygon whose triangle completion has the same orientation as the big triangle. Right: Put a black vertex to each polygon whose triangle completion has opposite orientation.

\footnotetext{
${ }^{19}$ The lines should be parallel with the boundary edges.

${ }^{20}$ This constraint is from the supersymmetric condition on the brane configuration.

${ }^{21}$ A bipartite network has vertices colored with the black or white, and there are no edges connecting the vertices with the same color.

${ }^{22}$ Roughly speaking, the network is the $(p, q)$ five brane web with two types of decoration on brane junctions.
} 
A bipartite network is formed by connecting the white vertex and black vertex if there is a common edge between two corresponding polygons (vertices with the same color are never connected). Moreover, an extra line is coming out of the boundaries for the boundary polygon. The network formed in this way is always bipartite but there may be vertices with only two edges. We can use the following moves to get rid of degree two vertices and get another bipartite network: Remove degree two vertices and then use the contraction to merge the line connecting the vertices with the same color. After this reduction, one can find a quiver from the network using the following rule: Assign a quiver node to each surface and the quiver arrows are determined by the black vertices, namely there is a clockwise closed circles around it, see Figure $30^{23}$.
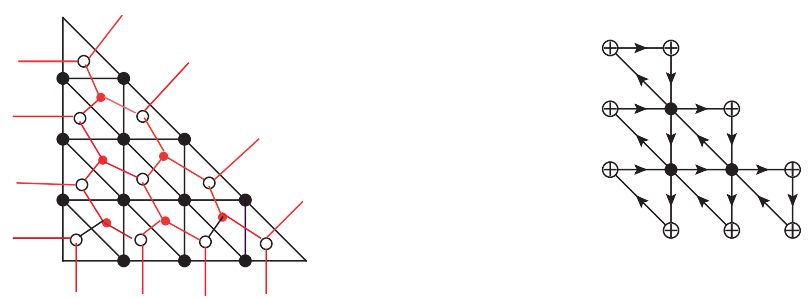

Figure 30: Left: The dot diagram and the bipartite network. Right: The quiver from the network: the black dot is the gauged node while the white one is the flavor node.

When there are more than three punctures, we start with a regular ideal triangulation and take a closed loop connecting all the punctures, and the boundary edges on this closed loop are decorated using the information of the Young Tableaux of the punctures in the same way as the triangle. The decoration of the other internal edges are automatically determined by the $S$ duality property which is studied in detail in [26]. After the decorations on the edges of all the triangles in the triangulation, one can do the tessellations on each triangle using the minimal polygon and find the network, quiver, etc. Many examples would be given in following sections..

Remember that we have taken a clockwise convention in doing the decoration of the boundary edges and the quiver arrows. One can take the

${ }^{23}$ The quiver diagram is very much like the dot diagram itself, however, usually more than one black dot represent a single quiver nodes. It is easy to read the quiver directly from the dot diagram without drawing the network after some practices. 
anti-clockwise orientation for the decoration and the choice of the quiver arrows, and they will give the equivalent result.

6.1.1. Quiver with potential and mutation. The BPS quiver of the $\mathcal{N}=2$ theory is derived from the bipartite network as described in some detail above. The potential $W$ can also be read pretty easily from the network, see Figure 31:

1. There is a potential term for each vertex of the network.

2. Each edge attached to this vertex represents a quiver arrow, and the potential term is a cyclic product of all the edges (the quiver arrows) attached on the vertex.
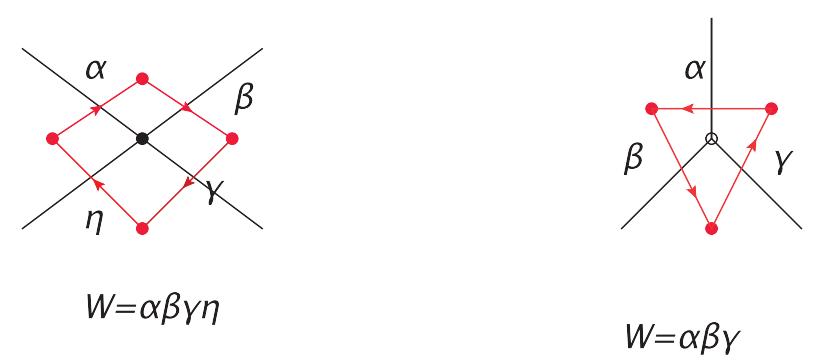

Figure 31: The rules for defining the potential for the quiver from bipartite network.

This assignment of the potential for the quiver is exactly like the rule for the corresponding $\mathcal{N}=1$ quiver gauge theory from the network. The BPS quiver is actually a pair $(Q, W)$ and the above rule makes sure that under the square move, the two quivers with potentials $(Q, W)$ and $\left(Q^{\prime}, W^{\prime}\right)$ are related by the mutation rules. This can be checked easily if the quiver mutations are represented by the square moves.

Now a major difference with the $A_{1}$ case is that not all of our quivers are acyclic, namely, there are two cycles in the quiver. To eliminate these 2cycles, one need the quadratic superpotential term associated with them, but these super potentials are sometimes missing. This fact is actually important for the consistency of our construction. Such quiver is not suitable for our later study of the spectrum using the quiver mutations which require the absence of 2-cycles, and one need to use the quiver representation theory directly to study them.

The BPS quiver $(Q, W)$ constructed above have the following features:

a. The total number of quiver nodes are equal to $2 n_{r}+n_{f}$, where $n_{r}$ is the rank of the gauge group and $n_{f}$ is the number of the mass deformations. 
b. The rank of the quiver is equal to $2 n_{r}$, this has been checked in many cases, it would be nice to have a general proof though.

c. The maximal number of arrows between two quiver nodes are two.

6.1.2. Two flips. Different triangulations of the same bordered Riemann surface are related by a sequence of local move called flip which relate two triangulations of the quadrilateral. In [34] we proved that if the dot diagram for the quadrilateral does not have the "bad" configuration shown in Figure 32 , the corresponding quivers of two triangulations (include the flavor nodes) are related by quiver mutation (or equivalently the two networks are related by square moves). Even for the "bad" corner, if we consider only the quiver nodes represented by the closed surfaces, the quivers from different triangulations are still related by quiver mutations. This is good enough for us, since only the quiver nodes associated with the closed surfaces are included into the BPS quiver.

Moreover, a sequence of quiver mutations acting only on the quiver nodes inside the triangle is also very useful for our later study of the BPS states counting. We call such quiver mutation sequences as "triangle" flip. In the following, we will provide some details on these two types of flips.

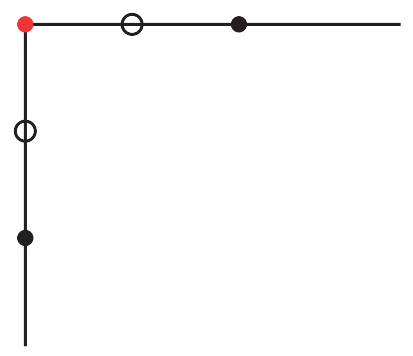

Figure 32: The network would be non-minimal if the boundary of the dot diagram has this form at any vertex.

Quadrilateral flip. This sequence of quiver mutations representing the quadrilateral flip are first discovered by Fock-Goncharov (FG) for the full puncture case in [33], and it is later generalized to general cases in [34]. The FG rules is best described using the dot diagram on the quadrilateral in which black dots are the quiver nodes. The quiver mutations representing flip can be done in $N-1$ steps: in step $i$, we inscribe a rectangle with lengths $i \times(N-i)^{24}$ along with the diagonal edge, i.e. inside the quadrilateral (the

\footnotetext{
${ }^{24}$ We ignore an irrelevant normalization factor here.
} 
sides with length $(N-i)$ is in parallel with the diagonal edge), then further decompose the rectangle into unit squares and we mutate the quiver nodes at the center of each little square at this step, see Figure 33 for the description of $A_{3}$ theory. The quiver after these sequence is the same as the quiver from the quadrilateral derived by flipping the diagonal edge of the original one. Notice that in each step the mutated quiver node has four quiver arrows. The total number of quiver mutations for one flip is

$$
N_{m}=\sum_{i=1}^{k} i(k-i)=\frac{1}{6}\left(N^{3}-N\right) .
$$

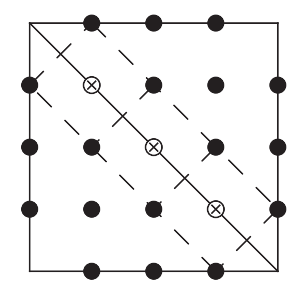

Step 1

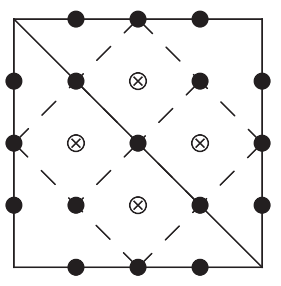

Step 2

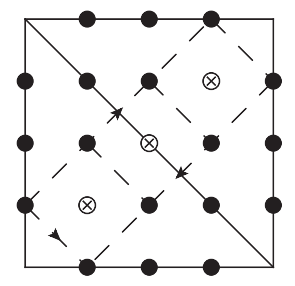

Step 3

Figure 33: Three steps for quiver mutations representing the flip for the quadrilateral with full punctures.

The quiver mutation sequences for the flip are found for the non-full puncture case if the glued network is minimal. In this case, usually a quiver node is represented by more than one black dots in the dot diagram. One still have the same rectangle and unit square decomposition for each step, but we only do the quiver mutations for the quiver nodes with four arrows. See Figure 34 for the quiver mutation sequences representing the flip of the quadrilateral with a non-full puncture puncture.

The number of quiver mutations realizing the flip in the case of the nonfull puncture can be counted explicitly if there is only one non-full puncture with partitions $\left[n_{1}, n_{2}, \ldots, n_{r}\right]$, and the total number of flips would be

$$
N_{m}-\sum_{i=1}^{r} \frac{1}{6}\left(n_{i}^{3}-n_{i}\right) .
$$

One can count the number of quiver mutations representing the flips case by case for more general quadrilaterals.

There is a very nice heuristic way of explaining why mutating the quiver nodes with four quiver arrows in each step. Let's regard the quiver from 

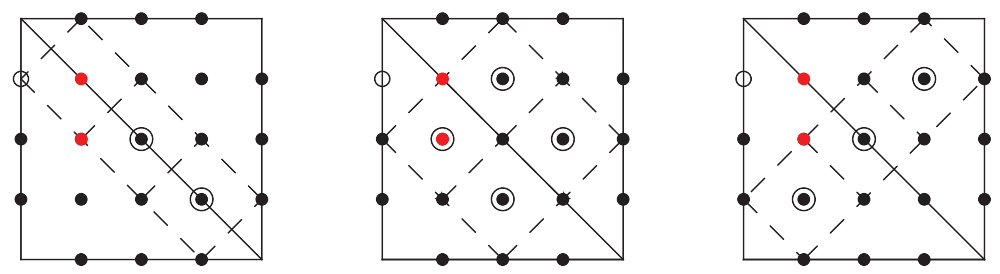

Figure 34: Three steps for quiver mutations representing the flip with nonfull puncture, and the two red nodes are identified as a single quiver node. In first step, the quiver nodes represented by the red dot has six arrows on it, so we do not mutate it; In second step, the quiver node represented by the red node has four arrows, and we mutate it.

the bipartite network as a four dimensional $\mathcal{N}=1$ quiver gauge theory by assigning $S U(N)$ to each quiver nodes (including the nodes associated with the open surfaces, which are the flavor groups.). Then the theory is anomaly free, and the flavors for each gauge group are $N_{f}=2 N, 3 N$, etc for the minimal network we considered thus far. Only for $N_{f}=2 N$ or the quiver nodes with four arrows, the gauge group is in conformal window, and one could do Seiberg duality (or quiver mutations) on it!

If the glued network for the quadrilateral is not minimal (there is a "bad" corner for the dot diagram and there are quiver nodes with only two quiver arrows on it.), the two networks associated with two triangulations of the quadrilateral are not related by square moves. We now state that the quivers associated with the closed surfaces (consider only the gauged quiver nodes) are still related by the quiver mutations.

One need special treatment for the quiver nodes with two arrows, namely those quiver nodes with $N_{f}=N$. By analogy with the Seiberg duality, one can still do the Seiberg duality on the quiver nodes with $N_{f}=N$ if we assign the rank $N$ to all the quiver nodes, but the rank of the gauge group becomes 0 after the Seiberg duality, so this quiver . node is frozen after the mutation. Then some other nodes may have five arrows where one arrow is connected with this zero rank gauge group, and we count the effective arrows of the quiver nodes by ignoring such type of arrows. The mutations sequences for the quadrilateral flip are found in the following steps: still use the inscribed rectangular in each step, and mutate the non-frozen quiver nodes with effective number of flavors $N_{f}=N$ or $N_{f}=2 N$. For example, the quiver mutations representing the flip of quadrilateral in Figure 35 are:

$$
\left(\mu_{4}\right),\left(\mu_{2}, \mu_{3}\right),\left(\mu_{1}, \mu_{5}\right) \text {. }
$$



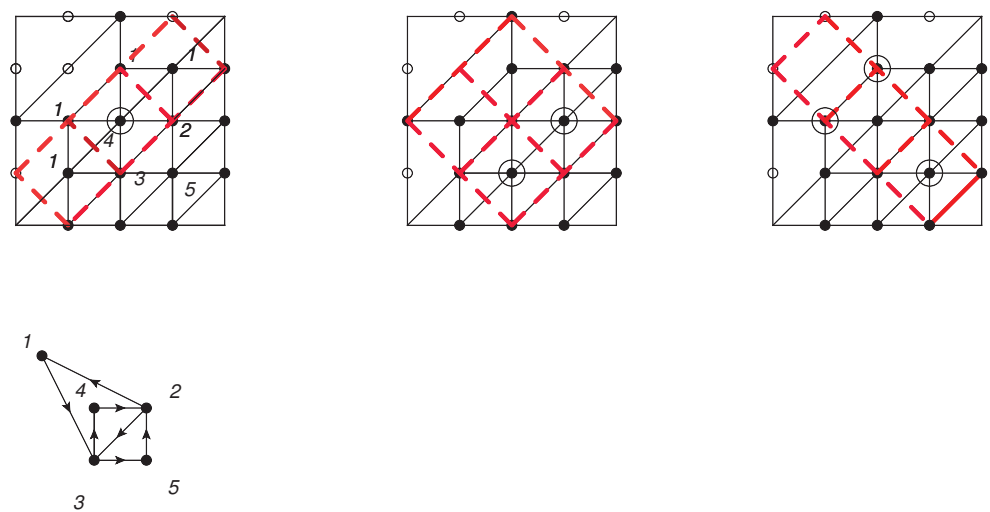

Figure 35: The quiver mutation sequences representing the flip for the quadrilateral with a "bad" corner.

Triangle flip. There are another flip called "triangle" flip acting only on the quiver nodes inside each triangle, which turns out to be very important for our later study of BPS states counting. Let's first study a triangle with three full punctures and we will describe the mutation sequences representing the triangle flip. To describe the sequences, it is useful to label the quiver nodes inside each triangle with three non-negative integers $(a, b, c)$ such that the distances to the sides $A, B, C$ satisfying the following relation (see Figure 36):

$$
a+b+c=N .
$$

Let's take side $C$ as a reference side, and the mutation sequences for the triangle flip could be described in $(N-2)$ steps: in each step $1 \leq i \leq N-2$, there are $N-1-i$ ordered sub steps: in each substep $(1 \leq j \leq N-1-i)$ starting with $j=1$, we mutate quiver nodes with label $(a, b, N-j-1)$. The total number of quiver mutations is

$$
N_{s}=\sum_{i=1}^{N-2} \sum_{j=1}^{N-i-1} j=\frac{1}{6}\left(N^{3}-3 N^{2}+2 N\right) .
$$

The triangle flip for the non-full puncture can be defined in a similar way. Let's start with the simplest case where the only non-full puncture has partition $\left[n_{1}, 1,1, \ldots, 1\right]$, see Figure 37 . The only difference from the full puncture case is that some of the inside nodes are missing (the boundary node are filling inside though which we will not count as the inside node.). 


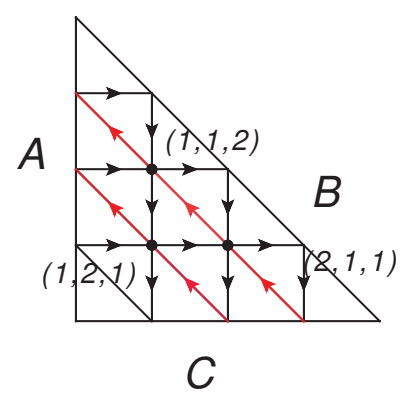

Figure 36: Each quiver node inside the triangle can be labeled by three integer numbers $(a, b, c)$ satisfying the relation $a+b+c=N$, here $a$ is the distance to the sides labeled by $A$, etc.



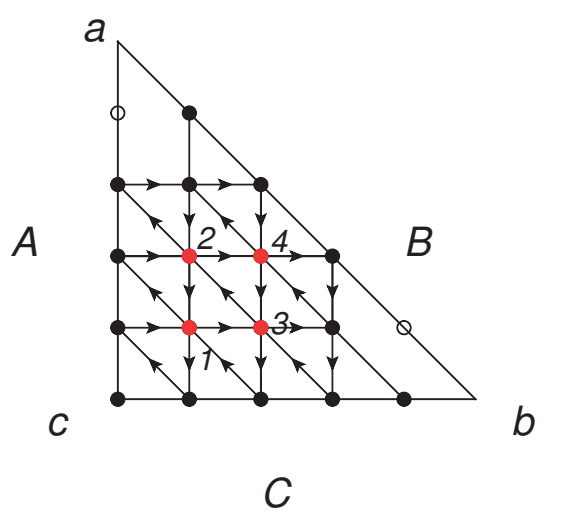

Figure 37: Left: A triangle with a non-full puncture for $A_{3}$ theory. Right: A triangle with two non-full punctures for $A_{4}$ theory.

There are two edges $(a b$ and $b c$ ) representing full punctures which could be used to glue other triangles, and we would like to describe the triangle flip relative to these two edges. The labeling of the remaining quiver nodes inside the triangle are the same as the full puncture case: they are labeled by $(a, b, c)$ with $1 \leq c \leq N-n_{1}-1$. The triangle flip with respect to edge $B=a b$ is represented by the following mutation sequences:

Step 1: Mutate quiver nodes with label $(a, i, c)$ starting from $i=N-2$ and ending with $i=1$.

Step 2: Mutate quiver nodes with label $(a, i, c)$ staring from $i=N-2$ and ending with $i=2$, here only the quiver nodes with coordinate $c \leq N-$ $n_{1}-2$ are mutated. 
Step j: Mutate quiver nodes with label $(a, i, c)$ staring from $i=N-2$ and ending with $i=j$, moreover, we only mutate quiver nodes with $c \leq$ $N-n_{1}-j$.

The above steps stop for $c=1$. According to above procedure, the "triangle" flip for the quiver on the left of Figure 37 has the following mutation sequences:

$$
\mu_{1}, \mu_{3}
$$

If there is another non-full puncture $b$ with partition $\left[m_{1}, 1,1, \ldots, 1\right]$, then some of the inner quiver nodes around puncture $b$ will be missing as shown in Figure 37. The "triangle" flip is implemented using the similar quiver mutation sequences:

Step j: Mutate quiver nodes with label $(a, i, c)$ staring from $i=N-2$ and ending with $i=j$, moreover, we only mutate quiver nodes with $c \leq$ $N-n_{1}-j$.

So the mutation sequences implementing the "triangle" flip of the right triangle is

$$
\left[\mu_{1},\left(\mu_{2}, \mu_{3}\right), \mu_{4}\right],\left[\mu_{1}, \mu_{3}\right]
$$

For more general configurations, one can also find similar mutation sequences for the triangle flip, since it seems that there is not a uniform formula, we choose not to present the details here.

\subsection{One boundary}

In this subsection, we are going to use maximal green mutation to find the finite spectrum of a theory whose BPS geometry has only one boundary, i.e. a disc with several marked points. The underlying $\mathcal{N}=2$ theory is a general Argyres-Douglas theory as discussed in detail in [25].

6.2.1. Disc with full punctures. Let's consider the higher rank generalization of a disc with $n$ marked points whose Young Tableaux are all full. The corresponding irregular singularities are identified in [35] and many other properties of these theories are studied in [25]. In particular, if there are $2(k+1)$ marked points, the theory is the so-called $\left(A_{N-1}, A_{k N-1}\right)$ theory ${ }^{25}$.

\footnotetext{
${ }^{25}$ The meaning of this label is that the BPS quiver is a direct product of the two corresponding Dynkin diagrams.
} 
We are going to use the quadrilateral flip and the triangle flip to find the finite BPS chamber of this class of theories. Let's define an internal edge as green if the quiver nodes on this edge are all green in the green mutation. The idea for finding the BPS spectrum is the following: Do the quadrilateral flips on the green edge in a random way, and stops if no internal green edge is left; finally the triangle flip are done for the quiver nodes inside each triangle. One could also do the triangle flip first and then do the quadrilateral flip, which is actually equivalent to the previous prescriptions. In the following, we are going to do the quadrilateral first, and let's discuss several simple examples in the following part of this subsection.

Example 6. Consider a disc with three full punctures, and the triangulation is just a triangle and the quiver is given in Figure 38. Since there is no quadrilateral here, we only need to do the triangle flip, and the maximal green mutation sequence is the same as the triangle flip. The number of BPS states in this chamber is

$$
N_{b p s}=N_{s}=\frac{1}{6}\left(N^{3}-3 N^{2}+2 N\right) .
$$

We conjecture that this is the minimal chamber, and there might be other chambers which could be found using random green mutations. The quiver shown in Figure 38 has $N=5$, and the maximal mutation sequences are

$$
\begin{aligned}
& \mu_{1},\left(\mu_{2}, \mu_{3}\right),\left(\mu_{4}, \mu_{5}, \mu_{6}\right), \\
& \mu_{1},\left(\mu_{2}, \mu_{3}\right), \\
& \mu_{1} .
\end{aligned}
$$

The mutation orders in each bracket is irrelevant.

The number of BPS states in the minimal chamber might be derived in the following simple way. Assume that the number of BPS states is a smooth function of $N$, and it scales as $N^{3}$. Since for $N \leq 2$, the theory is trivial and the number of BPS states is zero, the number of states in the minimal chamber takes the following form

$$
f(N)=a N(N-1)(N-2) .
$$

There is only one quiver node for $N=3$ and the number of BPS states for this theory is one. Using the simple data $f(3)=1$, we find $a=\frac{1}{6}$ which is exactly the answer derived from the mutations. Amazingly, we could get the right number of the BPS states by using this simple assumptions. 

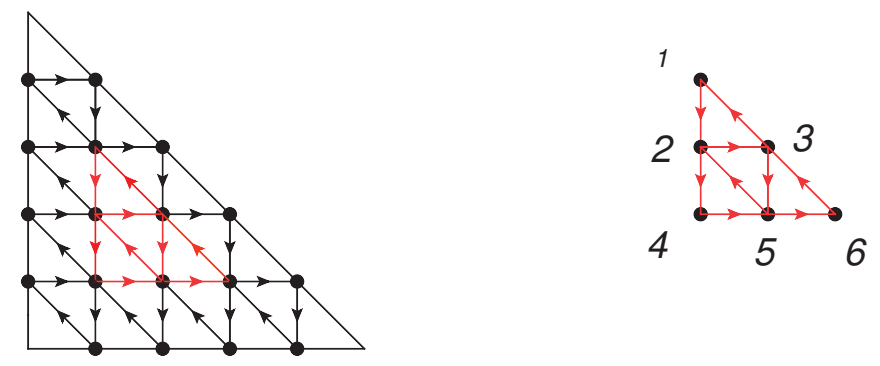

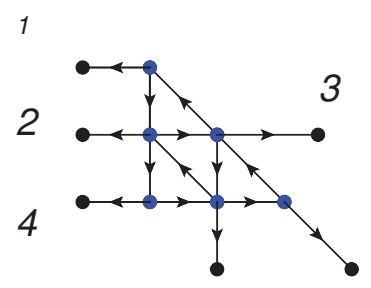

5
6

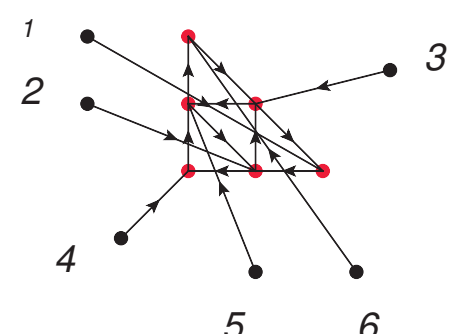

56

Figure 38: Top: The BPS quiver from the triangulation of a disc with three full punctures. Bottom: The initial and final configuration of the maximal green mutation.

Example 7. Consider a disc with four full punctures which is the BPS geometry for the $\left(A_{N-1}, A_{N-1}\right)$ theory. The maximal green mutation sequences involves one quadrilateral flip and two triangle flips, and the number of BPS states in this chamber is

$$
\begin{aligned}
N_{b p s} & =N_{m}+2 N_{s}=\frac{1}{6}\left(N^{3}-N\right)+\frac{1}{3}\left(N^{3}-3 N^{2}+2 N\right) \\
& =\frac{1}{2} N(N-1)^{2} .
\end{aligned}
$$

The charge vector of the BPS states can be easily found from the green mutation sequences.

Let's write explicitly the mutation sequences for $N=4$. It is important to track the position of the quiver nodes, i.e. whether it is inside the triangle or on the diagonal edge, since we need to do the triangle flip at the end. In the example, the quiver nodes 1,4,6 are grouped inside one triangle after the flip, and 3,5,7 is put inside another triangle. So the mutation sequences 
are

$$
\begin{aligned}
& \text { quadrilateral flip : }\left(\mu_{1}, \mu_{2}, \mu_{3}\right)\left(\mu_{4}, \mu_{5}, \mu_{6}, \mu_{7}\right)\left(\mu_{8}, \mu_{9}, \mu_{2}\right), \\
& \text { triangle flip } 1: \mu_{1},\left(\mu_{4}, \mu_{6}\right), \mu_{1}, \\
& \text { triangle flip } 2: \mu_{3},\left(\mu_{5}, \mu_{7}\right), \mu_{3} .
\end{aligned}
$$
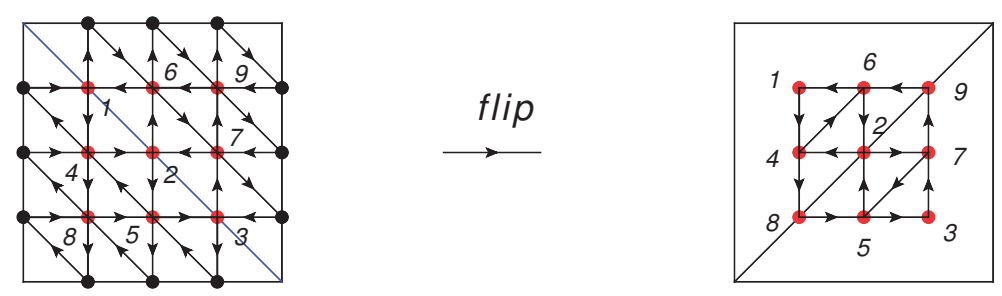

Figure 39: Left: The BPS quiver from a disc with four full punctures. Right: The position of the original quiver nodes after the quadrilateral flip.

Example 8. Consider a disc with five full punctures which is either a type I AD theory if $\mathrm{N}$ is even, or type II AD theory if $\mathrm{N}$ is odd [35]. One chamber involves two quadrilateral flips and three triangle flips, so the number of states are

$$
N_{1}=2 N_{m}+3 N_{s}=1 / 6 N(N-1)(5 N-4) .
$$

In another chamber, there are three big flips and also three triangles, and the number of the states are

$$
N_{2}=3 N_{m}+3 N_{s}=1 / 2 N(N-1)(2 N-1) .
$$

Let's give the mutation sequences for these two chambers for $N=3$ (higher rank cases are exactly the same). The mutation sequences involving two quadrilateral flips are (see Figure 40):

$$
\begin{aligned}
F_{1} & :\left(\mu_{2}, \mu_{3}\right),\left(\mu_{1}, \mu_{6}\right), \\
F_{2} & :\left(\mu_{4}, \mu_{5}\right),\left(\mu_{2}, \mu_{7}\right), \\
\text { triangle flip } & :\left(\mu_{3}, \mu_{4}, \mu_{5}\right) .
\end{aligned}
$$


The mutation sequences for the chamber involving three quadrilateral flips are (see Figure 41)

$$
\begin{aligned}
& F_{2}:\left(\mu_{4}, \mu_{5}\right),\left(\mu_{6}, \mu_{7}\right), \\
& F_{1}:\left(\mu_{2}, \mu_{3}\right),\left(\mu_{1}, \mu_{4}\right), \\
& F_{2}:\left(\mu_{6}, \mu_{7}\right),\left(\mu_{3}, \mu_{5}\right), \\
& \text { triangle flip }:\left(\mu_{2}, \mu_{6}, \mu_{7}\right) .
\end{aligned}
$$

Although the final quivers for two flip sequences are the same, the positioning of the original quiver nodes are quite different. For example, quiver nodes $(3,4,5)$ are inside the triangle in first chamber, but quiver nodes $(2,6,7)$ are playing this role in another chamber.

For a disc with $n+3$ full punctures, the minimal flip number are $n$ and the maximal number of flips are $\frac{n(n+1)}{2}$, so we find the following chambers:

$$
\begin{aligned}
& f_{\text {min }}=n N_{m}+(n+1) N_{s}, \\
& f_{\text {max }}=\frac{n(n+1)}{2} N_{m}+(n+1) N_{s} .
\end{aligned}
$$

Notice that $f_{\max }$ is not necessarily the number of BPS states in the maximal chamber.
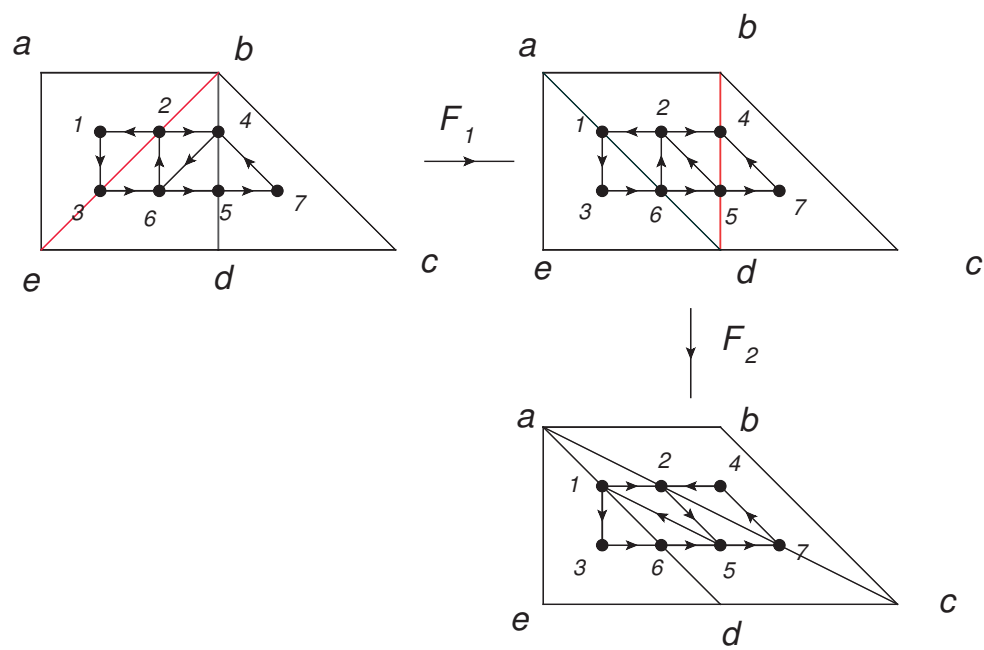

Figure 40: The flip sequences and the quiver positions for chamber found using two quadrilateral flips. 

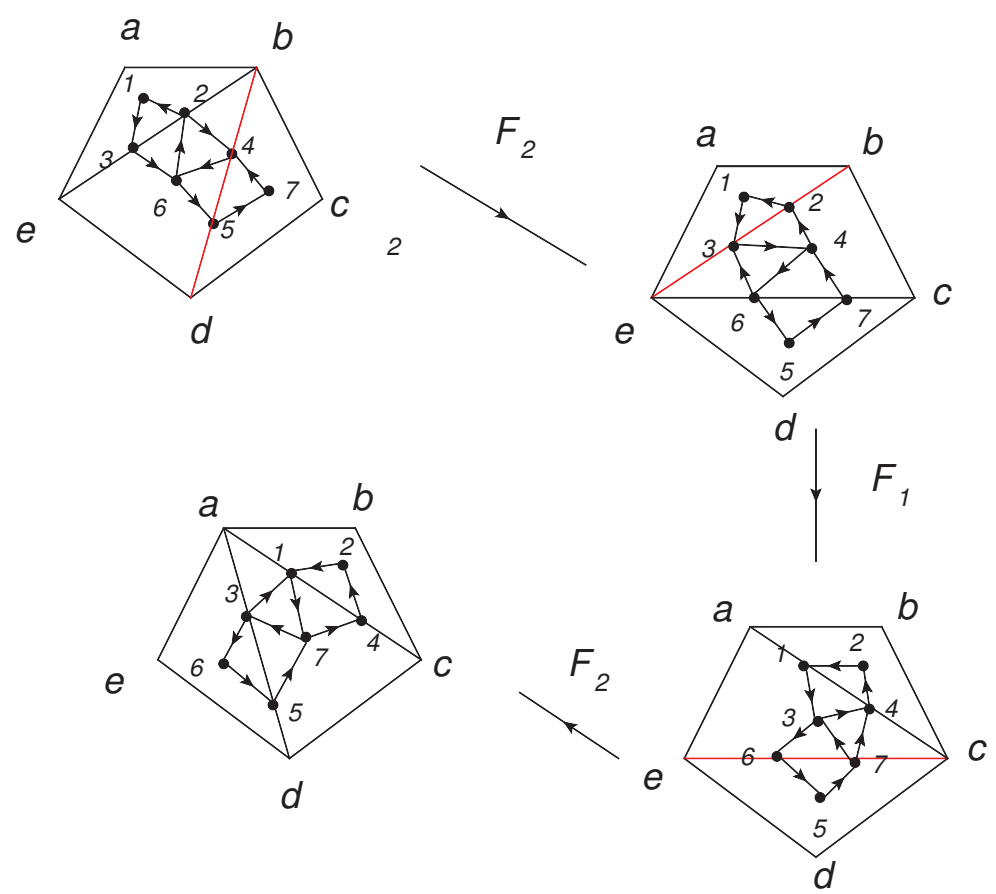

Figure 41: The flip sequences and the quiver positions for chamber involving three quadrilateral flips.

Generalized pentagon identity I. The pentagon identify for the $A_{1}$ theory is nicely represented by the five flip sequences of the pentagon which actually implies the basic quantum dilogarithm identify. As we show earlier using the maximal green mutation, this quantum dilogarithm identity is the wall crossing formula for the $A_{2}$ Argyres-Douglas theory: The product on one side of the identify corresponds to the chamber involving two flips $F_{1}, F_{2}$ while the product on the right is derived from the flip sequences $F_{2}, F_{1}, F_{2}$.

Now similar quantum dilogarithm identity can be found using wall crossing formula for the higher rank theory which is also represented by a pentagon. Now each quadrilateral flip is represented by a sequence of quiver mutations. However, unlike the $A_{1}$ case, the triangle flips are also important for finding the BPS spectrum. We need to list the charge vectors of two chambers for $N=3$, which are crucial for writing down the quantum dilogarithm identity. Let's call the chamber involving 2 flips (resp. 3 flips) 
as chamber I (chamber II), and the charge vectors for chamber I are

$$
\begin{aligned}
& F_{1}:\left(\gamma_{2}, \gamma_{3}\right),\left(\gamma_{1}+\gamma_{3}, \gamma_{2}+\gamma_{6}\right), \\
& F_{2}:\left(\gamma_{4}, \gamma_{5}\right),\left(\gamma_{5}+\gamma_{6}, \gamma_{4}+\gamma_{7}\right), \\
& \text { triangle flip }:\left(\gamma_{1}, \gamma_{6}, \gamma_{7}\right) ;
\end{aligned}
$$

Similarly, the charge vectors for chamber II are

$$
\begin{aligned}
& F_{2}:\left(\gamma_{4}, \gamma_{5}\right),\left(\gamma_{6}+\gamma_{5}, \gamma_{4}+\gamma_{7}\right), \\
& F_{1}:\left(\gamma_{2}+\gamma_{4}, \gamma_{3}+\gamma_{5}+\gamma_{6}\right),\left(\gamma_{1}+\gamma_{3}+\gamma_{5}+\gamma_{6}, \gamma_{2}+\gamma_{4}+\gamma_{7}\right), \\
& F_{2}:\left(\gamma_{3}, \gamma_{2}\right)\left(\gamma_{1}+\gamma_{3}, \gamma_{2}+\gamma_{6}\right), \\
& \text { triangle flip }:\left(\gamma_{1}, \gamma_{6}, \gamma_{7}\right) .
\end{aligned}
$$

A quantum dilogarithm function $E\left(y^{\gamma}\right)$ is associated for each BPS particle with charge $\gamma$ and all the BPS particle from one chamber form a ordered product. The wall crossing formula means that the two products from two chambers are the same. Using the mutation sequences and the charge vectors, we have

$$
\begin{aligned}
& E\left(y^{\gamma_{2}}\right) E\left(y^{\gamma_{3}}\right) E\left(y^{\gamma_{1}+\gamma_{3}}\right) E\left(y^{\gamma_{2}+\gamma_{6}}\right) E\left(y^{\gamma_{4}}\right) E\left(y^{\gamma_{5}}\right) E\left(y^{\gamma_{5}+\gamma_{6}}\right) \\
& E\left(y^{\gamma_{4}+\gamma_{7}}\right) E\left(y^{\gamma_{1}}\right) E\left(y^{\gamma_{6}}\right) E\left(y^{\gamma_{7}}\right) \\
= & E\left(y^{\gamma_{4}}\right) E\left(y^{\gamma_{5}}\right) E\left(y^{\gamma_{5}+\gamma_{6}}\right) E\left(y^{\gamma_{4}+\gamma_{7}}\right) E\left(y^{\gamma_{2}+\gamma_{4}}\right) E\left(y^{\gamma_{3}+\gamma_{5}+\gamma_{6}}\right) \\
& E\left(y^{\gamma_{1}+\gamma_{3}+\gamma_{5}+\gamma_{6}}\right) E\left(y^{\gamma_{2}+\gamma_{4}+\gamma_{7}}\right) E\left(y^{\gamma_{3}}\right) E\left(y^{\gamma_{2}}\right) E\left(y^{\gamma_{1}+\gamma_{3}}\right) \\
& E\left(y^{\gamma_{2}+\gamma_{6}}\right) E\left(y^{\gamma_{1}}\right) E\left(y^{\gamma_{6}}\right) E\left(y^{\gamma_{7}}\right) .
\end{aligned}
$$

It is interesting to note that the charge vectors from the triangle flip are the same for two chambers and they are living at the far left of the quantum dilogarithm product, so they do not participate in the wall crossing process and they can be cancelled out in the quantum dilogarithm identity ${ }^{26}$ :

$$
\begin{aligned}
& E\left(y^{\gamma_{2}}\right) E\left(y^{\gamma_{3}}\right) E\left(y^{\gamma_{1}+\gamma_{3}}\right) E\left(y^{\gamma_{2}+\gamma_{6}}\right) E\left(y^{\gamma_{4}}\right) E\left(y^{\gamma_{5}}\right) E\left(y^{\gamma_{5}+\gamma_{6}}\right) E\left(y^{\gamma_{4}+\gamma_{7}}\right) \\
= & E\left(y^{\gamma_{4}}\right) E\left(y^{\gamma_{5}}\right) E\left(y^{\gamma_{5}+\gamma_{6}}\right) E\left(y^{\gamma_{4}+\gamma_{7}}\right) E\left(y^{\gamma_{2}+\gamma_{4}}\right) E\left(y^{\gamma_{3}+\gamma_{5}+\gamma_{6}}\right) \\
& E\left(y^{\gamma_{1}+\gamma_{3}+\gamma_{5}+\gamma_{6}}\right) E\left(y^{\gamma_{2}+\gamma_{4}+\gamma_{7}}\right) E\left(y^{\gamma_{3}}\right) E\left(y^{\gamma_{2}}\right) E\left(y^{\gamma_{1}+\gamma_{3}}\right) E\left(y^{\gamma_{2}+\gamma_{6}}\right) .
\end{aligned}
$$

In fact, the cancellation of the BPS states from triangle flip in the quantum dilogarithm identity is true for any $N$ by explicitly checking the charge vectors. This is not so surprising from the quantum cluster algebra point

${ }^{26}$ We thank A.Neitzke for the helpful discussion on this point. 
of view, since the five quadrilateral flips would bring the coordinates back to their original values up to a permutation, so the quantum dilogarithm identity should only involve the mutations from the quadrilateral flips, see Figure 42. The mutation sequences and $c$ vector is shown in Table 11 for the five flips, and we can write the quantum dilogarithm identity

$$
\begin{aligned}
& E\left(y^{\gamma_{2}}\right) E\left(y^{\gamma_{3}}\right) E\left(y^{\gamma_{1}+\gamma_{3}}\right) E\left(y^{\gamma_{2}+\gamma_{6}}\right) E\left(y^{\gamma_{4}}\right) E\left(y^{\gamma_{5}}\right) E\left(y^{\gamma_{5}+\gamma_{6}}\right) E\left(y^{\gamma_{4}+\gamma_{7}}\right) \\
& E\left(y^{\gamma_{2}+\gamma_{6}}\right)^{-1} E\left(y^{\gamma_{1}+\gamma_{3}}\right)^{-1} E\left(y^{\gamma_{2}}\right)^{-1} E\left(y^{\gamma_{3}}\right)^{-1} E\left(y^{\gamma_{2}+\gamma_{4}+\gamma_{7}}\right)^{-1} \\
& E\left(y^{\gamma_{1}+\gamma_{3}+\gamma_{5}+\gamma_{6}}\right)^{-1} E\left(y^{\gamma_{3}+\gamma_{5}+\gamma_{6}}\right)^{-1} E\left(y^{\gamma_{2}+\gamma_{4}}\right)^{-1} E\left(y^{\gamma_{4}+\gamma_{7}}\right)^{-1} \\
& E\left(y^{\gamma_{5}+\gamma_{6}}\right)^{-1} E\left(y^{\gamma_{5}}\right)^{-1} E\left(y^{\gamma_{4}}\right)^{-1}=1
\end{aligned}
$$

which is the same quantum dilogarithm identity derived from the wall crossing formula.

\begin{tabular}{|c|c|c|c|}
\hline Flips & Mutation & $c$ vector & sign \\
\hline$F_{1}$ & $\left(\mu_{2}, \mu_{3}\right),\left(\mu_{1}, \mu_{6}\right)$ & $\left(\gamma_{2}, \gamma_{3}\right),\left(\gamma_{1}+\gamma_{3}, \gamma_{2}+\gamma_{6}\right)$ & $(+,+),(+,+)$ \\
\hline$F_{2}$ & $\left(\mu_{4}, \mu_{5}\right),\left(\mu_{2}, \mu_{7}\right)$ & $\left(\gamma_{4}, \gamma_{5}\right),\left(\gamma_{5}+\gamma_{6}, \gamma_{4}+\gamma_{7}\right)$ & $(+,+),(+,+)$ \\
\hline$F_{3}$ & $\left(\mu_{6}, \mu_{1}\right),\left(\mu_{5}, \mu_{3}\right)$ & $\left(-\gamma_{2}-\gamma_{6},-\gamma_{1}-\gamma_{3}\right),\left(-\gamma_{2},-\gamma_{3}\right)$ & $(-,-),(-,-)$ \\
\hline$F_{4}$ & $\left(\mu_{7}, \mu_{2}\right),\left(\mu_{1}, \mu_{4}\right)$ & $\left(-\gamma_{2}-\gamma_{4}-\gamma_{7},-\gamma_{1}-\gamma_{3}-\gamma_{5}-\gamma_{6}\right),\left(-\gamma_{3}-\gamma_{5}-\gamma_{6},-\gamma_{4}-\gamma_{2}\right)$ & $(-,-),(-,-)$ \\
\hline$F_{5}$ & $\left(\mu_{5}, \mu_{3}\right),\left(\mu_{6}, \mu_{7}\right)$ & $\left(-\gamma_{4}-\gamma_{7},-\gamma_{6}-\gamma_{5}\right),\left(-\gamma_{5},-\gamma_{4}\right)$ & $(-,-),(-,-)$ \\
\hline
\end{tabular}

Table 11: The mutation data for the five flips of the pentagon.

There is no need to calculate the cluster coordinates following the detailed mutation sequences, which would be a really tedious calculation. The easy way is to use the extended quiver introduced for the purpose of green mutation: we do green mutations for the first two flips and then red mutation for the next three flips, and every node is green again after five flips. There is a general theorem for the mutation sequence of an extended quiver: if the final quiver nodes are all green, then the final cluster coordinate is identical to the initial one up to a permutation. Using this theorem, we can easily verify that the cluster coordinates are back to themselves after five flips.

6.2.2. General $\left(\boldsymbol{A}_{N-1}, \boldsymbol{A}_{\boldsymbol{n} N-1+j}\right)$ theory. Other punctures are needed for the general Argyres-Douglas theory considered in [35]. Let's now consider general $\left(A_{N-1}, A_{n N-1+j}\right)$ theory with $0<j<N$, and the Stokes matrices analysis suggests that there are $j$ more marked points which are labeled by 


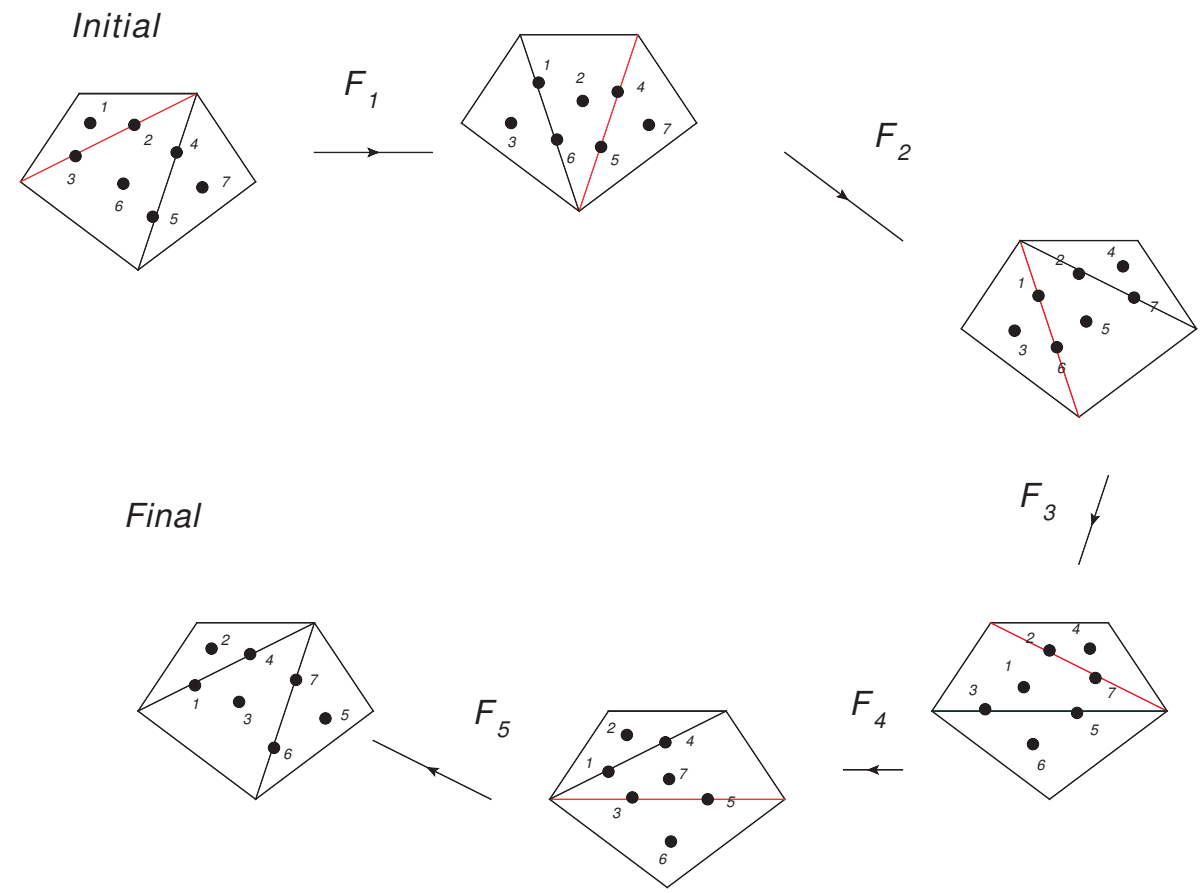

Figure 42: The pentagon identity for higher rank pentagon.

simple Young Tableaux. The number of marked points and their labels are

$$
\text { full : } 2(n+1) ; \quad \text { simple }: j
$$

The cyclic distribution of the marked points on the boundary of the disc is the following: there are $2(n+1)$ full punctures followed by $j$ simple punctures.

We will try to find the finite chamber using the following idea: do the quadrilateral flip sequences on the green edge and do the triangle flips at the end. Again, it is important to track the position of the quiver nodes, i.e. whether it is on the edge or inside the triangle, etc. The story is pretty the same as the full puncture cases, so we just give a simple example and the interested reader can do the similar exercises for the other BPS geometry.

Example 9. Let's consider a five punctured disc with four full punctures and one simple puncture of $N=4$. The triangulations and the quiver are shown in Figure 43. Using the two quadrilateral flips and triangle flips, one 
get the following maximal green mutation sequences:

$$
\begin{aligned}
& F_{1}:\left(\mu_{3}, \mu_{13}, \mu_{9}\right),\left(\mu_{2}, \mu_{10}, \mu_{8}, \mu_{12}\right),\left(\mu_{11}, \mu_{13}, \mu_{7}\right) \\
& F_{2}:\left(\mu_{4}, \mu_{5}, \mu_{6}\right),\left(\mu_{3}, \mu_{12}\right), \mu_{2}
\end{aligned}
$$

triangle flip $1: \mu_{9},\left(\mu_{8}, \mu_{10}\right), \mu_{9}$

$$
\text { triangle flip } 2:\left(\mu_{6},\left(\mu_{5}, \mu_{12}\right), \mu_{6}\right.
$$

For another chamber involving three quadrilateral flips (see Figure 44), the mutations sequences are,

$$
\begin{aligned}
& F_{2}:\left(\mu_{4}, \mu_{5}, \mu_{6}\right),\left(\mu_{7}, \mu_{12}\right), \mu_{8}, \\
& F_{1}:\left(\mu_{3}, \mu_{9}, \mu_{13}\right),\left(\mu_{2}, \mu_{10}\right), \mu_{11}, \\
& F_{2}:\left(\mu_{4}, \mu_{8}, \mu_{12}\right),\left(\mu_{5}, \mu_{7}, \mu_{9}, \mu_{13}\right),\left(\mu_{6}, \mu_{12}, \mu_{10}\right),
\end{aligned}
$$

triangle flip $1: \mu_{4},\left(\mu_{5}, \mu_{13}\right), \mu_{4}$,

(133) triangle flip $2: \mu_{8},\left(\mu_{7}, \mu_{9}\right), \mu_{8}$,

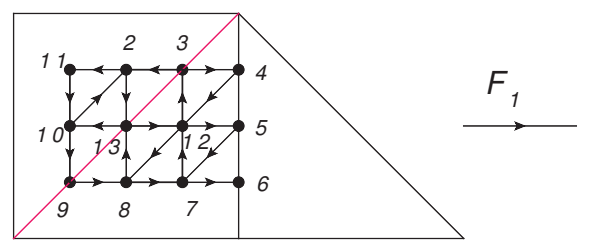

simple

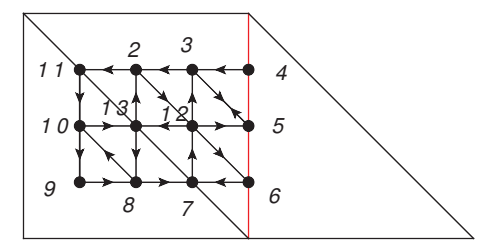

simple
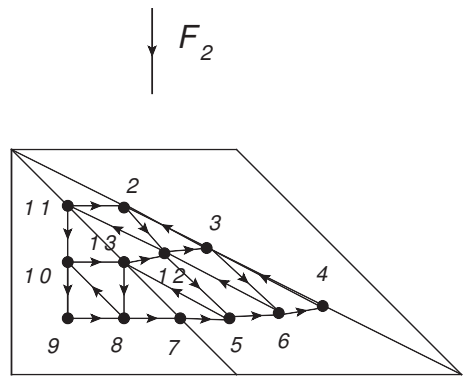

simple

Figure 43: The chamber involving two quadrilateral flips for the theory defined by a disc with four full punctures and one simple puncture. The quiver positions in each step are indicated. 

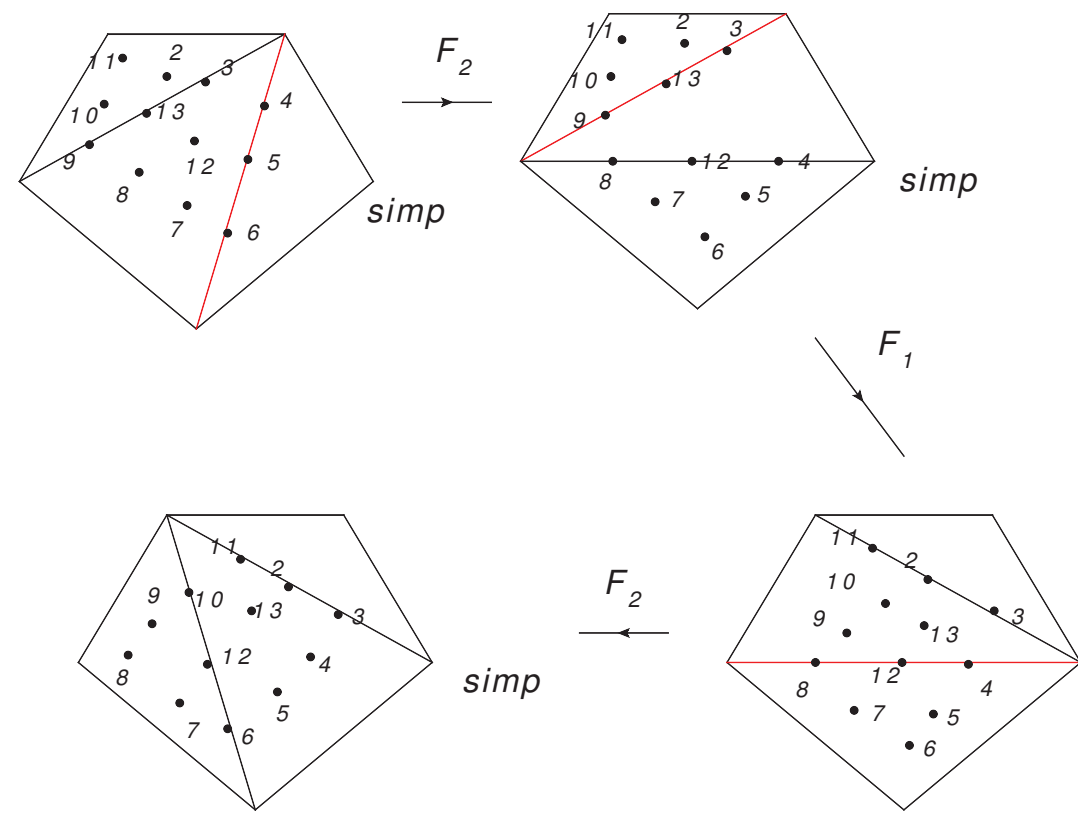

$\operatorname{simp}$

Figure 44: The chamber involving three quadrilateral flips for the theory defined by a disc with four full punctures and one simple puncture. The quiver positions in each step are indicated.

Generalized pentagon identity II. The pentagon identity can be generalized to the case where there are several full punctures and simple punctures. Similarly, one chamber comes from doing two quadrilateral flips while the other one is derived using three flips. The triangulation and quiver for the pentagon with only one simple puncture are shown in Figure 45 for $N=3$. The mutation sequences and the charge vectors for two chambers is shown in Table 12, and it is straightforward to write the following pentagon identity (again the terms from the triangle flip can be dropped out):

$$
\begin{aligned}
& E\left(y^{\gamma_{2}}\right) E\left(y^{\gamma_{6}}\right) E\left(y^{\gamma_{1}+\gamma_{6}}\right) E\left(y^{\gamma_{2}+\gamma_{5}}\right) E\left(y^{\gamma_{3}}\right) E\left(y^{\gamma_{4}}\right) E\left(y^{\gamma_{4}+\gamma_{5}}\right) \\
= & E\left(y^{\gamma_{3}}\right) E\left(y^{\gamma_{4}}\right) E\left(y^{\gamma_{4}+\gamma_{5}}\right) E\left(y^{\gamma_{2}+\gamma_{3}}\right) E\left(y^{\gamma_{5}+\gamma_{6}}\right) E\left(y^{\gamma_{1}+\gamma_{4}+\gamma_{5}+\gamma_{6}}\right) \\
& E\left(y^{\gamma_{2}}\right) E\left(y^{\gamma_{5}}\right) E\left(y^{\gamma_{2}+\gamma_{5}}\right) E\left(y^{\gamma_{1}+\gamma_{6}}\right) .
\end{aligned}
$$

The interested reader can work out the pentagon identity for other combinations of full punctures and simple punctures.

Sink-source sequences. The BPS quiver for $\left(A_{N-1}, A_{k-1}\right)$ from our network construction is mutation equivalent to the quiver formed by a product 


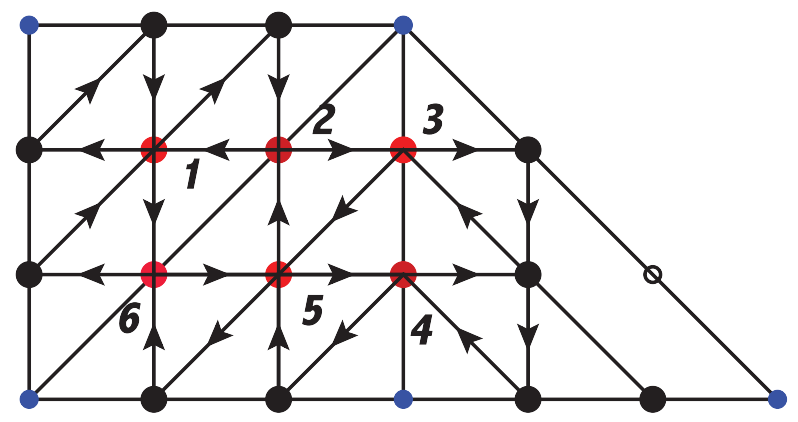

Figure 45: The quiver from pentagon with four full punctures and one simple puncture, here $N=3$.

\begin{tabular}{|c|c|}
\hline & Chamber I \\
\hline Mutations & $F_{1}:\left(\mu_{2}, \mu_{6}, \mu_{1}, \mu_{5}\right), F_{2}:\left(\mu_{3}, \mu_{4}, \mu_{2}\right), T_{f l i p}:\left(\mu_{6}, \mu_{4}\right)$ \\
\hline Charges & $\left(\gamma_{2}, \gamma_{6}, \gamma_{1}+\gamma_{6}, \gamma_{2}+\gamma_{5}\right),\left(\gamma_{3}, \gamma_{4}, \gamma_{4}+\gamma_{5}\right),\left(\gamma_{1}, \gamma_{5}\right)$ \\
\hline & Chamber II \\
\hline Mutations & $F_{2}:\left(\mu_{3}, \mu_{4}, \mu_{5}\right), F_{1}:\left(\mu_{2}, \mu_{6}, \mu_{1}\right), F_{2}:\left(\mu_{3}, \mu_{5}, \mu_{4}, \mu_{6}\right), T_{f l i p}:\left(\mu_{5}, \mu_{3}\right)$ \\
\hline Charges & $\left(\gamma_{3}, \gamma_{4}, \gamma_{4}+\gamma_{5}\right),\left(\gamma_{2}+\gamma_{3}, \gamma_{5}+\gamma_{6}, \gamma_{1}+\gamma_{4}+\gamma_{5}+\gamma_{6}\right),\left(\gamma_{2}, \gamma_{5}, \gamma_{2}+\gamma_{5}, \gamma_{1}+\gamma_{6}\right),\left(\gamma_{1}, \gamma_{5}\right)$ \\
\hline
\end{tabular}

Table 12: The mutation data for two chambers of a pentagon with one simple puncture.

of $A_{N-1}$ and $A_{k-1}$ Dynkin diagram which gives the name for the theory in [16]. The quiver mutation sequences for relating our quiver and the square $\left(A_{N-1}, A_{k-1}\right)$ quivercan be readily found. Let's give an example for $N=5$ and $k=5$ whose quiver is derived from a disc with four full punctures. The quiver mutations transforming the quiver from the triangulation to the $\left(A_{4}, A_{4}\right)$ form are:

$$
\mu_{1},\left(\mu_{2}, \mu_{3}\right), \mu_{4},\left(\mu_{5}, \mu_{6}\right)
$$

Let's now focus on the $\left(A_{N-1}, A_{k-1}\right)$ quiver and choose a convention that the horizon direction is $\left(A_{k-1}\right)$ quiver. The special feature of the subquiver in the horizontal direction and the vertical direction are that they are bipartite, and the quiver arrows form cyclic squares, see the left quiver in Figure 46. Moreover, if a quiver node is a sink node in the horizontal direction, it would be a source node in the vertical direction. Let's denote the signature of a quiver node as $(-,+)$ if it is a sink in the horizontal direction and source in 

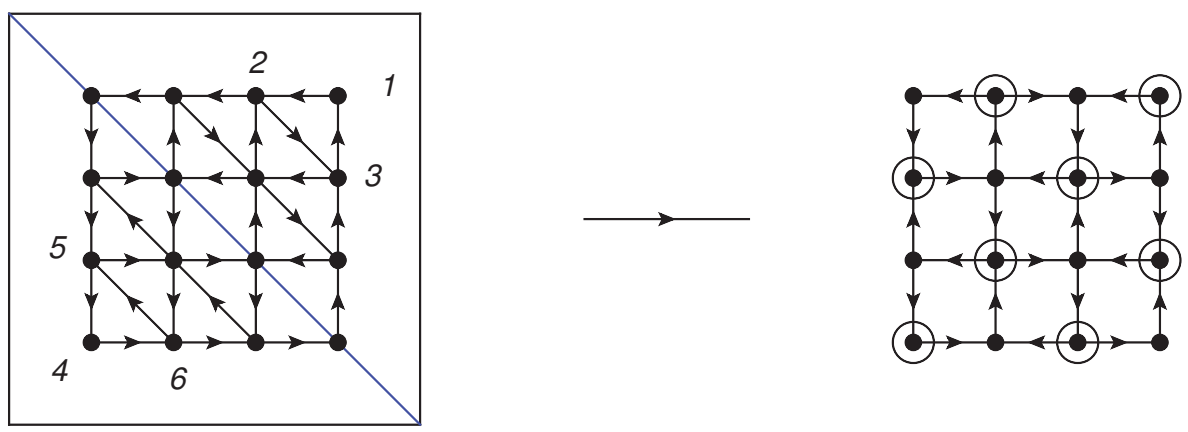

Figure 46: The mutations transforming the quiver from the triangulation to the $\left(A_{4}, A_{4}\right)$ quiver.

the vertical direction. Define the quiver mutation sequences

$$
\begin{aligned}
& \tau_{1}=\mu_{-+}, \\
& \tau_{2}=\mu_{+-},
\end{aligned}
$$

here $\mu_{-+}$(resp. $\mu_{+-}$) is the quiver mutation on all the quiver nodes with signature $(-,+)$ (resp. $(+,-))$. It can be checked explicitly that $\left(\tau_{1}\right)^{h^{\prime}}$ and $\left(\tau_{2}\right)^{h}$ give two maximal green mutation sequences, where $h^{\prime}$ (resp. $h$ ) is the Coxeter number for group $A_{k-1}\left(A_{N-1}\right)$. Such sequences are found in [16], here the charge vectors can be easily found using the maximal green mutations. When $N=2$, the quiver is just the bipartite quiver of $A_{k-1}$ Dynkin diagram. The Coxeter number of $A_{1}$ group and $A_{k-1}$ group are two and $k$, and $\tau_{2}^{2}$ is the source green mutation sequence and gives a total of $k-1$ states which is actually the minimal chamber; $\tau_{1}^{k}$ is the sink green mutation sequence and gives a total of $\frac{k(k-1)}{2}$ states which is the maximal chamber. In general, one of the green mutation sequence is the minimal chamber, but the other one might not be the maximal chamber.

6.2.3. Other $\mathrm{AD}$ theories from the disc. The $(A, A)$ type $\mathrm{AD}$ theories involve only the full and simple punctures, and more general punctures appear for other type of AD theories considered in detail in [35]. There are two more general classes whose BPS geometry involves a single disc. The type II AD theory which also has only full punctures and the simple punctures, but the boundary nodes of the simple puncture are gauged (we include the node on the edge of the simple puncture into our BPS quiver). The strategy of finding the BPS spectrum is the following: doing the quadrilateral flips and 
triangle flips as we did for the $(A, A)$ theory, and then do arbitrary green mutation sequences involving the boundary nodes!

The BPS geometry of type III AD theory also involves a single disc, and the Young Tableaux for the marked points are more fruitful. The definition of type III AD theory includes a sequences of Young Tableaux which satisfies the following condition

$$
Y_{n} \subset Y_{n-1} \cdots \subset Y_{1}
$$

where $Y_{1}$ is taken to be the full Young Tableaux so that the BPS quiver has the simple description [35], and $Y_{i-1}$ is derived by further decomposing the columns of $Y_{i}$. The BPS geometry is a disc with $2(n-1)$ Young Tableaux: $Y_{n}, Y_{n-1}, \ldots, Y_{2}, Y_{n}, \ldots, Y_{2}$ are arranged in cyclic order. The strategy for finding the finite states chamber for type III AD theory is the following: do the mutation sequences corresponding to flips; and then do the green mutations on the edge nodes and internal nodes repeatedly. This mutation sequences are very useful since many superconformal field theory engineered using Riemann surface and regular punctures has an realization as type III AD theory.

Example 10. Let's take $Y_{3}=[2,4], Y_{2}=[2,1,1,1,1]$, and $Y_{1}=[1,1,1$, $1,1,1]$, and the dot diagram and quiver are shown in Figure 47. This theory is in fact equivalent to the one engineered using $A_{1}$ theory on a sphere with five punctures. After doing the flip sequences, one need to do green mutations on the edge nodes and internal nodes repeatedly. One maximal green mutation sequences are

$$
\begin{aligned}
& \left(\mu_{2}, \mu_{3}, \mu_{4}\right),\left(\mu_{6}, \mu_{7}, \mu_{8}, \mu_{9}\right),\left(\mu_{1}, \mu_{5}, \mu_{3}\right), \\
& \left(\mu_{2}, \mu_{7}, \mu_{6}, \mu_{4}, \mu_{8}, \mu_{9}, \mu_{4}, \mu_{6}, \mu_{7}, \mu_{2}\right)
\end{aligned}
$$

For the theory engineered using six dimensional $A_{1}$ theory compactified on a sphere with $k$ punctures, there is a higher rank realization using type III AD theory with the following Young tableaux

$$
Y_{3}=[2, k-1], \quad Y_{2}=[2,1,1, \ldots, 1], \quad Y_{1}=[1,1,1, \ldots, 1]
$$

The BPS geometry is a fourth punctured disc with marked points $Y_{3}, Y_{2}, Y_{3}$, $Y_{2}$. It is not hard to find the finite chamber using the mutation sequences representing the flips of this quadrilateral. 



Figure 47: The triangulation of the fourth punctured disc with ordered puncture $\left(Y_{3}, Y_{2}, Y_{3}, Y_{2}\right)$. The bottom part shows the triangulation of $A_{1}$ theory on a sphere with 5 punctures whose quiver is equivalent to the disc configuration.

\subsection{Riemann surface without punctures}

6.3.1. Annulus with one marked point on boundary. The next simplest BPS geometry is the annulus with one marked point on each boundary, which represents a gauge group coupled with two matter sectors. To find the finite spectrum, one can not not do random quadrilateral flips on the green edges. This is the case where the flip sequences found in $A_{1}$ theory plays an important role here: we do the flip sequences found from the finite spectrum of the corresponding $A_{1}$ theory with the same type of BPS geometry, and finally do the green mutations on the quiver nodes inside each triangle.

Example 11. The BPS geometry is an annulus with a simple puncture on each boundary from which a quiver can be found from the dot diagram on the triangulation, see Figure 48B. We do the flip sequences found from the $A_{1}$ version: first mutate edge 1 and then mutate edge 2. Here some new features appear: one need to do more rounds of mutations. The flip one edge 1 is realized by the quiver mutations on node $1,2,3$, etc. The maximal green 
A
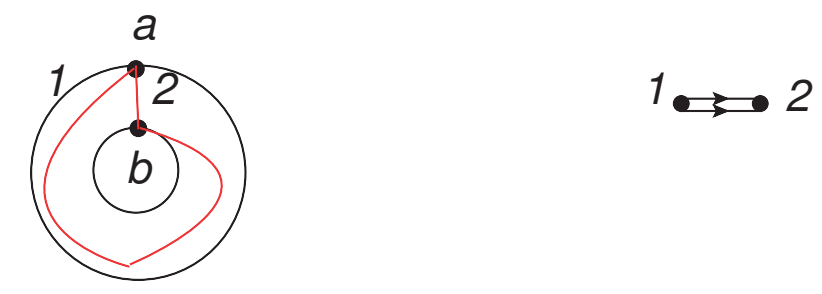

B

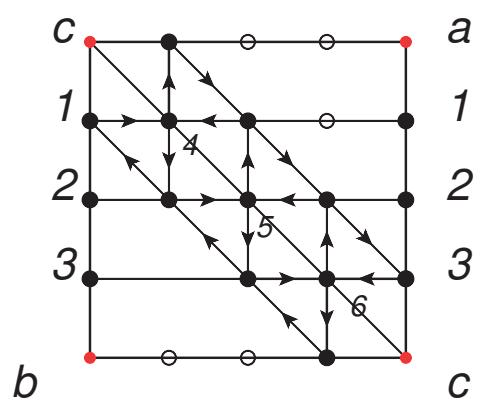

C

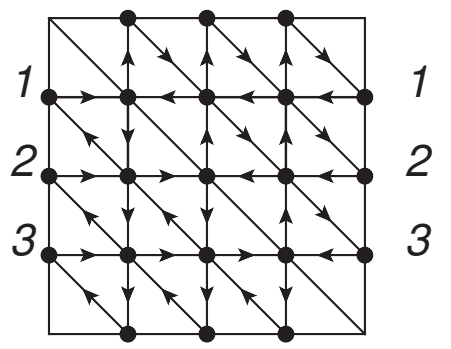

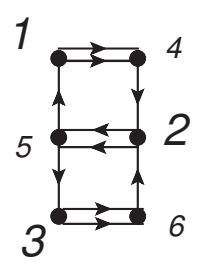

$C$

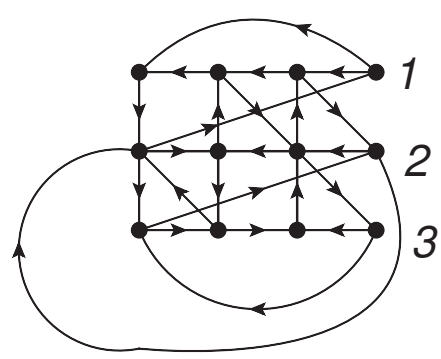

Figure 48: A: The triangulation and quiver for the annulus with one marked point on each boundary, here $N=2$. B: The quiver for annulus where each boundary has a simple puncture, here $N=4$. C: The quiver with two full punctures on each boundary of the annulus, here $N=4$.

mutation sequences for the pure $S U(4)$ theory is

$$
\left(\mu_{1}, \mu_{2}, \mu_{3}\right),\left(\mu_{4}, \mu_{5}, \mu_{6}\right),\left(\mu_{1}, \mu_{2}, \mu_{3}\right),\left(\mu_{4}, \mu_{5}, \mu_{6}\right) .
$$


The order of mutations in each bracket is not important since the corresponding quiver nodes are disconnected. There are 12 total BPS states, and in general this chamber for pure $\mathrm{SU}(\mathrm{N})$ theory has the following number of BPS states

$$
N_{b p s}=N(N-1),
$$

In fact, the BPS quiver is a sink-source product of affine $A_{1}$ diagram and $A_{N-1}$ Dynkin diagram, and the mutation sequence is just a generalization of the bipartite quiver. The result is the same as found in [21, 68].
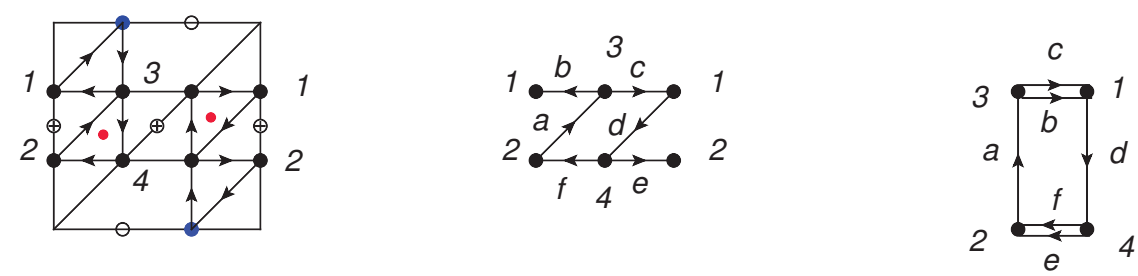

$W=a c d f+a b d e$

$W=a c d f+a b d e$

Figure 49: The triangulation, BPS quiver and the superpotential of the pure $S U(3)$ theory.

Unique finite chamber for pure $S \boldsymbol{U}(\boldsymbol{N})$ theory: Let's now argue that the finite chamber for $S U(N)$ theory is unique: the above chamber is the only finite chamber. Let's do the analysis for the $S U(3)$ theory, and the BPS quiver and the superpotential can be easily found from the network construction, see Figure 49. The potential is shown in Figure ??, and the $F$ term equations from the quiver are

$$
c d f+b d e=0, f a c+e a b=0 \quad d e a=0, \quad d f a=0, \quad a c d=0, \quad a b d=0
$$

The maximal green mutation sequences are

$$
\left(\mu_{3}, \mu_{4}\right),\left(\mu_{1}, \mu_{2}\right),\left(\mu_{3}, \mu_{4}\right) \text {. }
$$

Let's now give a proof that this is the unique sequences for finding a finite chamber using the quiver representation theory. The dimension vector for 
two indecomposable representations representing vector bosons are

$$
P_{1}=(1,0,1,0), \quad P_{2}=(0,1,0,1),
$$

The two corresponding subquivers are the affine $A_{1}$ quiver and the sink nodes are 1 and 2, therefore we have to mutate node 3 or node 4 in the first step (the analysis of the quiver representation theory is the same as the BPS quiver of the pure $S U(2)$ theory.). If we mutate node 3 first, and the node 1 and node 2 would form an affine $A_{1}$ quiver (both of them are green), with node 1 as the sink node, and they represent the vector boson too. So we can not mutate on node 2 and node 1 in this second step, and we have to mutate node 4 in second step. Similar analysis can be done for the following quiver mutation sequences ${ }^{27}$, and the above mutation sequences are the unique one to find a finite spectrum. The proof can be easily generalized to higher rank pure $S U(N)$ theory, and our conclusion is that there is only one finite chamber.

Example 12. The BPS quiver for $S U(N)$ with $N_{f} \leq 2 N$ theory is shown in Figure 50. The quiver has a main body formed by the quiver of pure $S U(N)$ theory, and each additional flavor adds a new vertex and new triangle to the quiver. The strategy of finding the finite spectrum is very simple: doing the quiver mutation sequences for the pure SYM and then do random green mutations on the extra flavor nodes.

Let's list one maximal green mutation sequences for the $S U(4)$ gauge theory with $N_{f}=1$ (the BPS quiver is shown in Figure 50B):

$$
\begin{aligned}
& \left(\mu_{1}, \mu_{2}, \mu_{3}\right),\left(\mu_{4}, \mu_{5}, \mu_{6}\right),\left(\mu_{1}, \mu_{2}, \mu_{3}\right),\left(\mu_{4}, \mu_{5}, \mu_{6}\right), \\
& \mu_{7}, \mu_{4}, \mu_{1}, \mu_{5}, \mu_{2}, \mu_{6}, \mu_{3} .
\end{aligned}
$$

Similarly, for $S U(4)$ gauge theory with $N_{f}=2$ (the BPS quiver is shown in Figure 50C), a maximal green mutation sequence is

$$
\begin{aligned}
& \left(\mu_{1}, \mu_{2}, \mu_{3}\right),\left(\mu_{4}, \mu_{5}, \mu_{6}\right),\left(\mu_{1}, \mu_{2}, \mu_{3}\right),\left(\mu_{4}, \mu_{5}, \mu_{6}\right), \\
& \mu_{7}, \mu_{4}, \mu_{1}, \mu_{5}, \mu_{2}, \mu_{6}, \mu_{3}, \\
& \mu_{8}, \mu_{2}, \mu_{6}, \mu_{1}, \mu_{5}, \mu_{7}, \mu_{4} .
\end{aligned}
$$

In general, each extra flavor would need $2 N-1$ extra green mutations (one need to mutate the extra node once and all the other quiver nodes

${ }^{27}$ The crucial point is the following: if there are two green quiver nodes connected by two arrows, the sink node can not be mutated. 
$A$

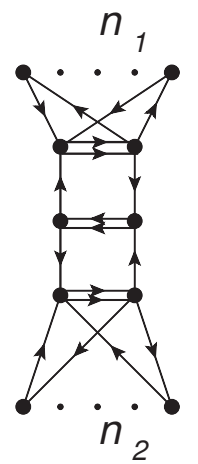

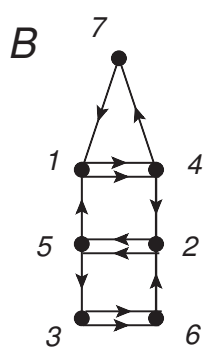

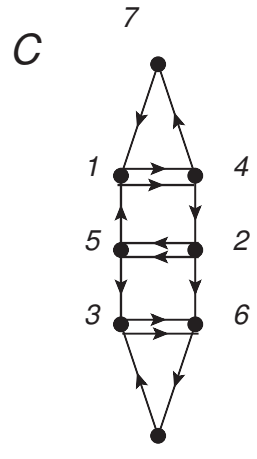

8

$N_{f}=2$

Figure 50: The BPS quiver for $S U(4)$ theory with $n_{1}+n_{2}$ fundamental flavors.

for the pure $S U(N)$ theory once), and the finite chamber has the following number of states

$$
N(N-1)+(2 N-1) N_{f}
$$

This is in agreement with the result presented in [21]. Notice that there are other finite chambers if we start mutating the extra quiver nodes first, and the above number is not necessarily the minimal chamber for $S U(N)$ with $N_{f}$ flavors, since the number might be smaller if we start with a quiver which is mutation equivalent to the above one.

Example 13. If there is one simple puncture on one boundary and one full puncture on another boundary. When $N$ is even, the underlying $\mathcal{N}=2$ theory is actually a linear quiver:

$$
S U(N)-S U(N-2)-\cdots-S U(2)
$$

and it is the following linear quiver

$$
S U(N)-S U(N-2)-\cdots-S U(3)-1
$$

when $N$ is odd. 

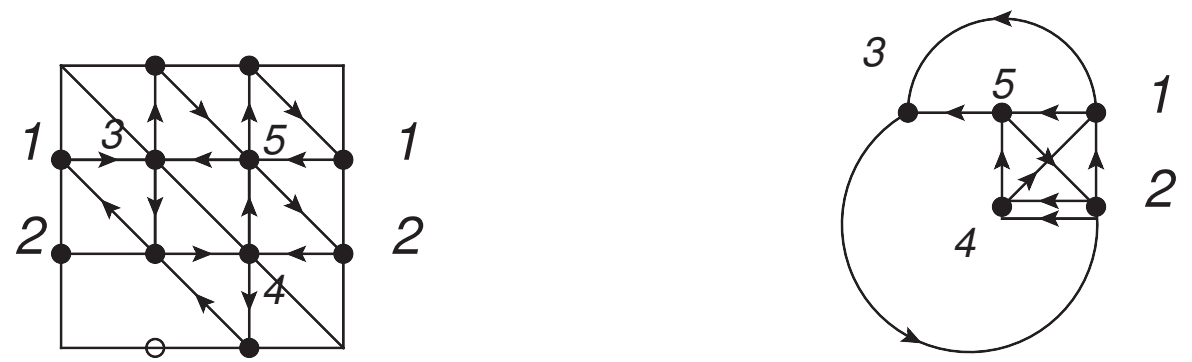

Figure 51: The dot diagram for the annulus with one simple and one full puncture on each boundary, and the quiver is shown on the right.

Let's look at the example shown in Figure 51 with $N=3$, which geometry actually represents $S U(3)$ gauge theory with $N_{f}=1$. The maximal green mutation sequence from our prescriptions are

$$
\begin{aligned}
& F_{1}:\left(\mu_{1}, \mu_{2}, \mu_{5}\right), \\
& F_{2}:\left(\mu_{3}, \mu_{4}, \mu_{1}\right),
\end{aligned}
$$

Triangle : $\left(\mu_{3}, \mu_{5}\right)$.

The first two steps are the mutation sequences for the quadrilateral flips, and the last step is some kind of generalized triangle flip.

In general, there are are two quadrilateral flips and each flip involves $\frac{1}{2} N(N-1)$ steps. Finally one need to do the triangle flip on one triangle with three full punctures which gives $\frac{1}{6} N(N-1)(N-2)$ mutations, moreover, one need to do green mutations on the quiver nodes on the edges which involve $2(N-2)$ mutations.

Example 14. Let's now consider an annulus with two full punctures on each boundary. The underlying $\mathcal{N}=2$ theory is actually a linear quiver

$$
\begin{gathered}
S U(2)-S U(4)-\cdots-S U(N-2)-S U(N)-S U(N-2) \\
-\cdots-S U(4)-S U(2)
\end{gathered}
$$

when $N$ is even, and it is the linear quiver

$$
\begin{gathered}
1-S U(3)-\cdots S U(N-2)-S U(N)-S U(N-2) \\
-\cdots-S U(3)-1 .
\end{gathered}
$$


when $N$ is odd. The BPS geometry and the quiver is shown in Figure 48. There are quiver nodes inside each triangle, so we need to do triangle flip first, and then do two flips, so the total number of BPS states in this chamber is

$$
N_{\text {bps }}=2 N_{s}+2 N_{m}=\frac{1}{3} N(N-1)(2 N-1) .
$$

Some funny numerology. We have found some sequences of numbers which seems to have the following pattern on dependence of rank $N$ if all the punctures are simple or full:

a. The number of BPS states is at most cubic in $N$.

b. The number has factor $N(N-1)$.

The first fact might be related to $N^{3}$ behavior of six dimensional $(2,0)$ theory; the latter fact is a manifestation that when $N=0$ and $N=1$, there is no BPS states. These two facts suggest that the dependence on $N$ has very fewer parameters and elegant form. For example, if the number of BPS states with a fixed BPS geometry has $N^{2}$ behavior, then the number of BPS states has only one free parameter and it must take the following form

$$
f_{1}(N)=a N(N-1) .
$$

If there is a $N^{3}$ behavior for the number of BPS states, then the formula has a maximal three parameters and take the following simple form

$$
f_{2}(N)=N(N-1)(a N+b) .
$$

So it is easy to determine those parameters using the result of the lower rank theory. For instance, when there are two simple punctures, the number of BPS states under large $N$ has only one parameter $a$ which can be fixed as 1 by substituting the result $f_{1}(2)=2$. Similarly, if there are two full punctures, using the result $f_{2}(2)=2$ and $f_{2}(3)=10$, we find $a=\frac{2}{3}$ and $b=-\frac{1}{3}$ which reproduce the results from explicit counting.

6.3.2. More marked points and more boundaries. The situation is quite similar for the Riemann surface with more marked points and more boundaries. One simply recalled the flip sequences from the maximal green mutations of the corresponding $A_{1}$ theory which is described in detail in previous section, and then use the same flip sequences to the higher rank theory.

Example 15. Let's consider an annulus with one full puncture on one boundary, and two simple punctures on the other boundary. The triangulation and the quiver is shown in Figure 52. The minimal flip sequences for 
the $A_{1}$ theory is $F_{1}, F_{2}, F_{3}$, and one maximal green mutation sequences for $N=3$ are

$$
\begin{aligned}
F_{1} & :\left(\mu_{a}, \mu_{b}, \mu_{c}\right), \\
F_{2} & :\left(\mu_{f}, \mu_{g}, \mu_{a},\right. \\
F_{3} & :\left(\mu_{d}, \mu_{e}, \mu_{f}\right), \\
\text { triangle flip } & :\left(\mu_{d}, \mu_{c}\right) .
\end{aligned}
$$
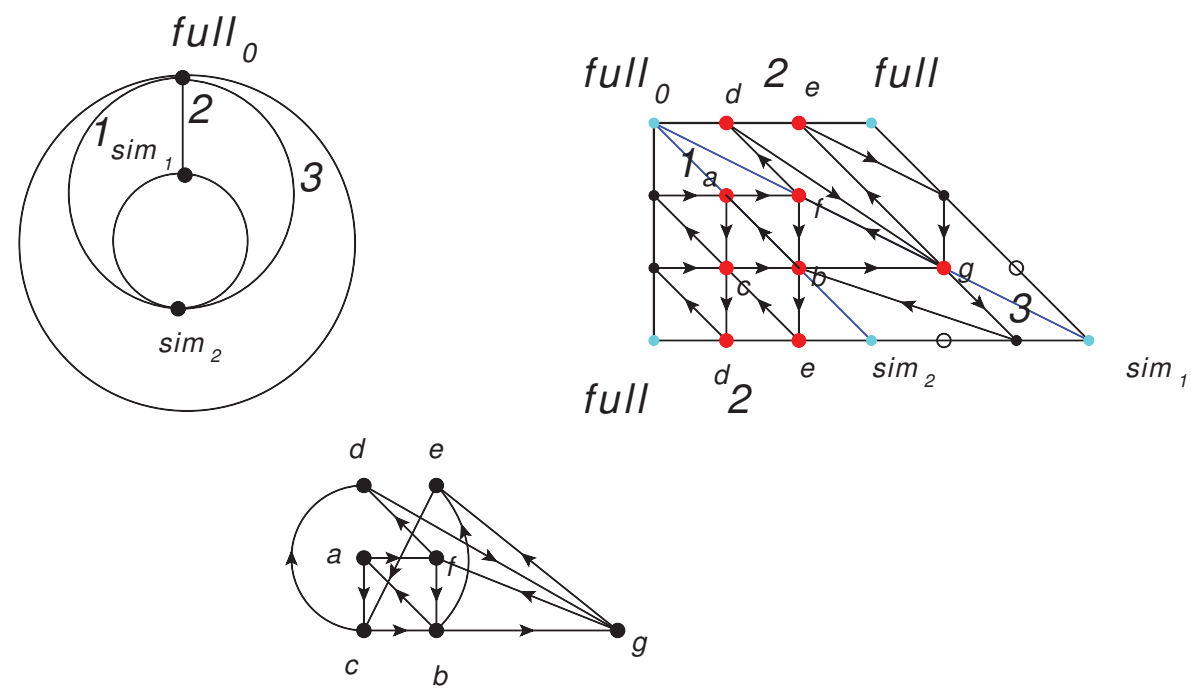

Figure 52: The triangulation and dot diagram of annulus with two simple punctures on one boundary and one full puncture on another boundary. The quiver is also shown.

\subsection{Once punctured disc}

This is the case where the geometric knowledge of the $A_{1}$ theory can not be generalized since the quiver mutation will create self-folded triangle, and furthermore one need to do mutation on quiver nodes corresponding to an edge of the self-folded triangle. The mutation on this special edge does not have a good meaning in the higher rank case, i.e. we do not know the mutation sequences for such flip. So one need some new clues about the mutation sequences. The irregular realization is crucial in providing the clues about the mutation sequences. 
Let's consider the simplest BPS geometry of this type: a once punctured digon and all punctures are full. The experimental rule we find is the following: first mutate the quiver nodes inside each triangle and use the mutation sequences corresponding to the triangle flip; then mutate the quiver nodes on two edges; and then do the quiver mutations on the inside nodes again, etc. This strategy is pretty successful in finding the maximal green mutation sequences.

Example 16. The BPS geometry is the once punctured digon which represents an AD theory, and we take all the puncture as full. This type of AD theory is actually isomorphic to the $A_{N-2}$ theory compactified on a sphere with $N$ simple punctures and a full puncture. The theory in one duality frame has the Lagrangian description and is given by

$$
1-S U(2)-S U(3)-\cdots-S U(N-1)-N
$$

The theory is isomorphic to $S U(2)$ with four flavors when $N=3$. We conjecture that the minimal chamber has the following number of BPS states:

$$
N_{b p s}=N(N-1)^{2} \text {. }
$$

The method for finding the spectrum is the following: first do the triangle flips for all the quiver nodes inside two triangles, and then do the quiver mutations on the quiver nodes on the edge. One need to mutate multiple times in this fashion, and the shape of the quivers inside the quiver nodes might change, however, the triangle flips can be done for each connected subquiver inside the triangle. The maximal green mutation sequences for $N=4$ is

$$
\begin{aligned}
& \left(\mu_{7}, \mu_{8}, \mu_{9}, \mu_{7} ; \mu_{10}, \mu_{11}, \mu_{12}, \mu_{10}\right),\left(\mu_{1}, \mu_{2}, \mu_{3}, \mu_{4}, \mu_{5}, \mu_{6}\right), \\
& \left(\mu_{10}, \mu_{11}, \mu_{12}, \mu_{7}, \mu_{8}, \mu_{9}\right),\left(\mu_{2}, \mu_{3}, \mu_{5}, \mu_{6}\right), \\
& \left(\mu_{10}, \mu_{11}, \mu_{12}, \mu_{7}, \mu_{8}, \mu_{9}\right),\left(\mu_{2}, \mu_{3}, \mu_{5}, \mu_{6}\right) .
\end{aligned}
$$

This type of sequences can be seen from the irregular realization of the same theory, i.e. it can be realized by a rank $2 N-1$ theory compactified on a sphere with an order three irregular singularity with the type

$$
Y_{3}=[N, N-1], \quad Y_{2}=[N-1,1,1, \ldots, 1], \quad Y_{3}=[1,1, \ldots, 1]
$$

The BPS geometry is a fourth punctured disc with cyclic ordered marked points $\left(Y_{3}, Y_{2}, Y_{3}, Y_{2}\right)$, and we draw the dot diagram and the quiver for $N=4$ 

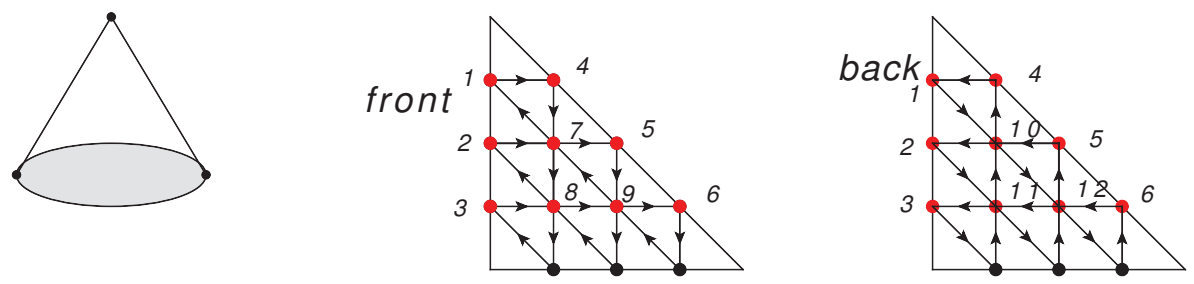

Figure 53: The triangulation and quiver for the once punctured digon, all the punctures are full. There are two triangles and the quiver is derived by identifying the quiver nodes on the edges of the triangulation.

in Figure 54, which is the same as the quiver using the $A_{N-1}$ representation. In the irregular realization, all the mass nodes are put on the diagonal edge, and one can start doing triangle flip first, and then do the quadrilateral flip, which gives the above sequences.
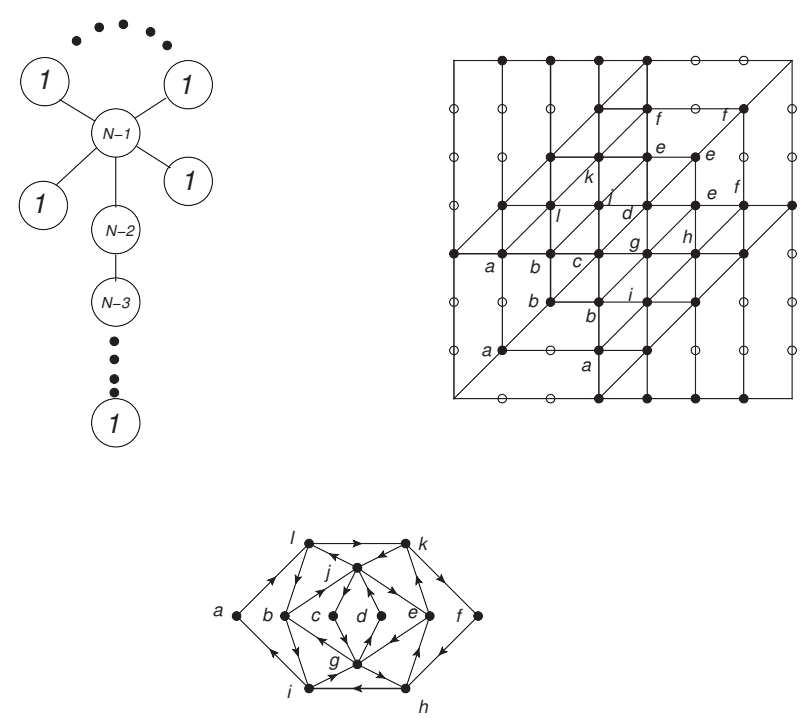

Figure 54: Top: The irregular realization can be seen from the 3d mirror of this theory, and we draw the dot diagram. Bottom: The quiver from this realization, which is the same as in Figure (53).

For other type of theories, one do random quadrilateral flips and if we encounter the above once punctured digon, we do the above specific mutation sequences and then keep going until there is no green edge left, as usual, we would need to do triangle flip at the end. In this way, we can find many maximal mutation sequences. 


\subsection{Closed Riemann surface}

We consider four dimensional superconformal field theory derived by compactifying six dimensional $A_{N-1}$ theory on a Riemann surface with regular punctures. The scaling dimensions of Coulomb branch operators are integer and usually there are marginal coupling constants. We are going to find the maximal mutation sequences for these class of theories.

$\boldsymbol{T}_{\boldsymbol{N}}$ theory. : The $T_{N}$ theory is realized as the sphere with three full punctures. The BPS quiver is derived from the triangulation of the three punctures sphere: there are two triangles with the same boundaries. We conjecture that the minimal chamber has the following number of states:

$$
N_{b p s}=2 N(N-1)^{2}
$$

The reasoning is still based on our conjecture that the BPS states is of the order $N^{3}$, and the BPS states is zero for $N=0$ and $N=1$, so the BPS states has the following form

$$
f(N)=N(N-1)(a N+b)
$$

Using the result for $N=2$ and $N=3$ found in [21], we get the conjectured form. There are 72 states for $N=4,160$ states for $N=5$ which we have checked explicitly. The charge vectors and the phase order can be found from the maximal green mutations. Interestingly, this formula gives the correct answer for $N=2$ even if the mutation method can not be applied to that quiver.

Example 17. The triangulations and dot diagram for $T 3$ and $T 4$ theory is shown in Figure 55. The maximal green mutations for $T 3$ theory is

$$
\begin{aligned}
& \left(\mu_{7}, \mu_{8}\right),\left(\mu_{1}, \mu_{2}, \mu_{3}, \mu_{4}, \mu_{5}, \mu_{6}\right) \\
& \left(\mu_{7}, \mu_{8}\right),\left(\mu_{1}, \mu_{2}, \mu_{3}, \mu_{4}, \mu_{5}, \mu_{6}\right) \\
& \left(\mu_{7}, \mu_{8}\right),\left(\mu_{1}, \mu_{2}, \mu_{3}, \mu_{4}, \mu_{5}, \mu_{6}\right)
\end{aligned}
$$


Front
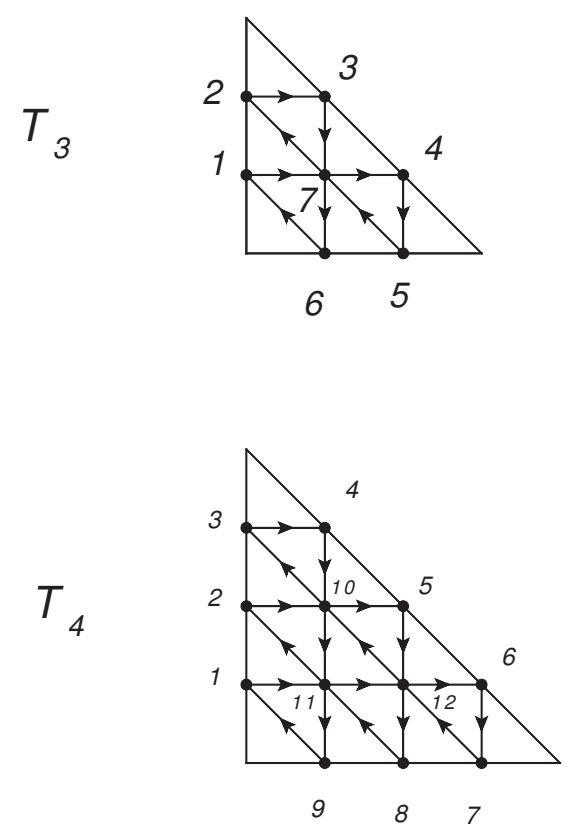

Back
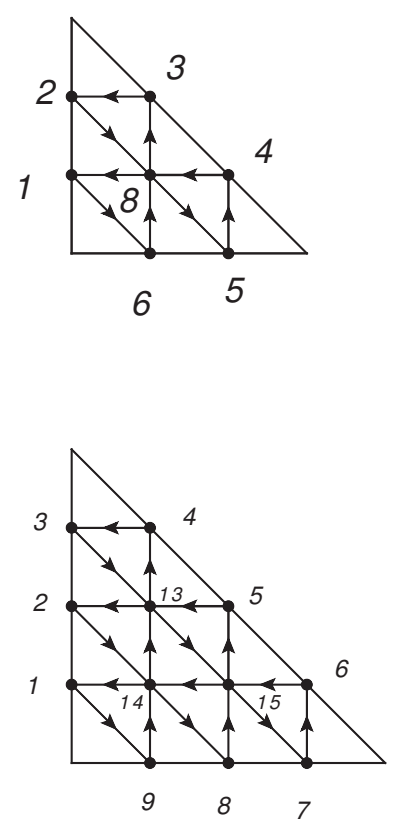

Figure 55: The triangulation and the quivers for $T 3$ and $T 4$ theory.

The Green mutation sequences for the $T 4$ theory is

$$
\begin{aligned}
& {\left[\mu_{10},\left(\mu_{11}, \mu_{12}\right), \mu_{10}\right],\left[\mu_{13},\left(\mu_{14}, \mu_{15}\right), \mu_{13}\right]} \\
& \mu_{1}, \mu_{2}, \mu_{3}, \mu_{4}, \mu_{5}, \mu_{6}, \mu_{7}, \mu_{8}, \mu_{9} \\
& \mu_{10}, \mu_{11}, \mu_{12}, \mu_{13}, \mu_{14}, \mu_{15} \\
& \mu_{1}, \mu_{2}, \mu_{3}, \mu_{4}, \mu_{5}, \mu_{6}, \mu_{7}, \mu_{8}, \mu_{9} \\
& \mu_{10},\left(\mu_{11}, \mu_{12}\right), \mu_{10}, \mu_{13},\left(\mu_{14}, \mu_{15}\right), \mu_{13} \\
& \mu_{1}, \mu_{2}, \mu_{3}, \mu_{4}, \mu_{5}, \mu_{6}, \mu_{7}, \mu_{8}, \mu_{9} \\
& \mu_{10}, \mu_{11}, \mu_{12}, \mu_{13}, \mu_{14}, \mu_{15} \\
& \mu_{2}, \mu_{5}, \mu_{8} \\
& \mu_{10},\left(\mu_{11}, \mu_{12}\right), \mu_{10}, \mu_{13},\left(\mu_{14}, \mu_{15}\right), \mu_{13} \\
& \mu_{1}, \mu_{3}, \mu_{4}, \mu_{6}, \mu_{7}, \mu_{9}
\end{aligned}
$$

The basic rule is to mutate the internal nodes using the triangle flip such that no green nodes left, then mutate all the boundary nodes. One 
need to do such sequences in several rounds. The motivation is coming from the irregular realization of the $T_{N}$ theory. For example, the $T 4$ theory has another realization using rank 9 theory compactified on a sphere with the following order three type III irregular singularity

$$
Y_{3}=[6,4], \quad Y_{2}=[4,3,3], \quad Y_{1}=[1,1,1, \ldots, 1]
$$

and the quiver for this irregular singularity is a fourth punctured sphere with cyclic ordered punctures $\left[Y_{3}, Y_{2}, Y_{3}, Y_{2}\right]$, the BPS quiver is shown in Figure 56. It is easy to check that the two quivers from different realization are the same. Now all the mass nodes in $A_{3}$ realization are living on the
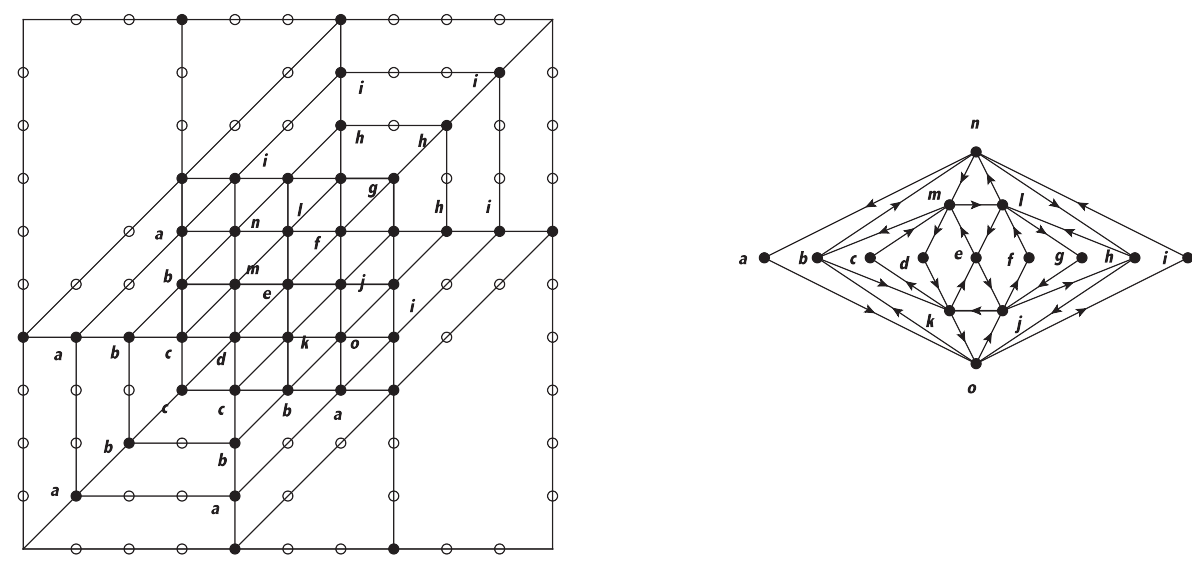

Figure 56: The irregular realization of $T 4$ theory.

single diagonal edges of the irregular realization, and the quiver nodes inside triangles are living inside the triangles in the irregular realization too. The mutation sequences for the flip is mutating the quiver nodes on the boundary nodes first, and then mutating the quiver nodes inside two triangles. We need to do more than one rounds following our early study on this type of $\mathrm{AD}$ theories. That is how we find the above mutation sequences.

Example 18. $S U(N)$ with $N_{f}=2 N$ : This is represented by a sphere with two full punctures and two simple punctures. The triangulation and the quiver is shown in Figure 57. We find a finite chamber with following number of states:

$$
N_{b p s}=2 N(2 N-1)
$$


The mutation sequences for $S U(3)$ with 6 flavor is the following

$$
\begin{aligned}
& \mu_{1}, \mu_{2}, \mu_{3}, \mu_{4}, \mu_{5}, \mu_{6} \\
& \mu_{7}, \mu_{8}, \mu_{9}, \mu_{10} \\
& \mu_{1}, \mu_{2}, \mu_{3}, \mu_{4}, \mu_{5}, \mu_{6} \\
& \mu_{7}, \mu_{8}, \mu_{9}, \mu_{10} \\
& \mu_{1}, \mu_{2}, \mu_{3}, \mu_{4}, \mu_{5}, \mu_{6} \\
& \mu_{7}, \mu_{8}, \mu_{9}, \mu_{10}
\end{aligned}
$$

In general, one first mutate the $2 N$ quiver nodes on the boundary, and then $(2 N-2)$ quiver nodes on the internal edges. The number of mutations for one cycle is $4 N-2$, and one need to do $N$ cycles, so the total number of states are $N(4 N-2)$.
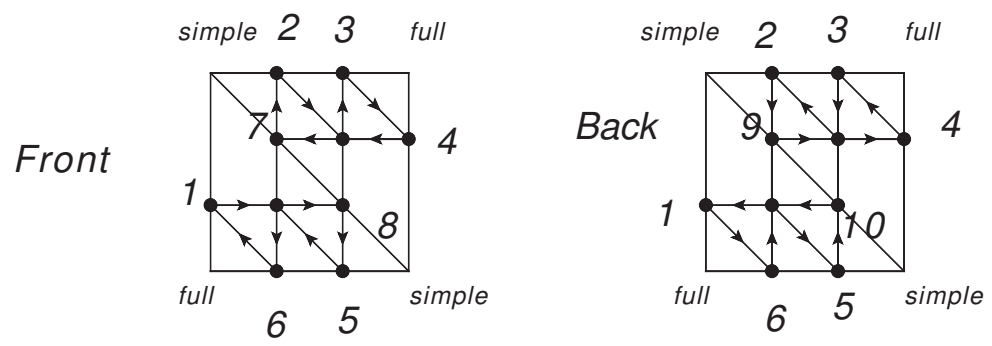

Figure 57: The triangulation and quivers for the theory defined by sphere with two full punctures and two simple punctures.

For more general theory defined by a sphere with punctures, it is possible to find a realization using the irregular singularity. Such realization is always possible if all the Young Tableaux has the form $\left[n_{1}, 1,1,1, \ldots, 1\right]$, in particular, it is possible if all the punctures are full. The irregular realization uses a even higher rank six dimensional group and a type III irregular singularity with an order 3 pole, see [35] for the exact map. The BPS geometry is a disc with four punctures. One can use the mutation sequences for the flip to find the maximal green mutations.

Example 19. Let's consider the theory defined by $A_{2}$ theory compactified on a sphere with four full punctures. This is a superconformal gauge theory with a $S U(3)$ group coupled to two $T_{3}$ theory. The three dimensional mirror for this theory is shown in Figure 58 from which we can read an irregular realizations, and we take the following rank 8 realization.

$$
Y_{3}=[6,3], \quad Y_{2}=[3,2,2,2], \quad Y_{1}=[1,1,1,1,1,1,1,1,1] .
$$


The BPS quiver is shown in Figure 58. This quiver is the same as the quiver derived from the triangulations of the fourth puncture sphere, we indicate the specific triangulations on Figure 58. The interested reader can check that the two quivers are indeed the same. Basically, the flavor nodes of the theory lives on the diagonal edge of the triangulation of fourth punctured disc. One of the maximal green mutation sequences which has 60 states is the following

$$
\begin{aligned}
& \left(\mu_{a}, \mu_{b}, \mu_{c}, \mu_{d}, \mu_{e}, \mu_{f}, \mu_{g}, \mu_{h}\right),\left(\mu_{m}, \mu_{n}, \mu_{o}, \mu_{i}, \mu_{j}, \mu_{k}\right),\left(\mu_{p}, \mu_{l}\right), \\
& \left(\mu_{a}, \mu_{b}, \mu_{c}, \mu_{d}, \mu_{e}, \mu_{f}, \mu_{g}, \mu_{h}\right),\left(\mu_{p}, \mu_{n}, \mu_{m}, \mu_{o}, \mu_{p}, \mu_{n}\right), \\
& \left(\mu_{j}, \mu_{l}, \mu_{i}, \mu_{k}, \mu_{j}, \mu_{l}\right),\left(\mu_{a}, \mu_{b}, \mu_{c}, \mu_{d}, \mu_{e}, \mu_{f}, \mu_{g}, \mu_{h}\right),\left(\mu_{p}, \mu_{n}, \mu_{j}, \mu_{l}\right), \\
& \left(\mu_{a}, \mu_{d}, \mu_{e}, \mu_{h}\right),\left(\mu_{m}, \mu_{o}, \mu_{i}, \mu_{k}\right),\left(\mu_{p}, \mu_{n}, \mu_{j}, \mu_{l}\right) .
\end{aligned}
$$
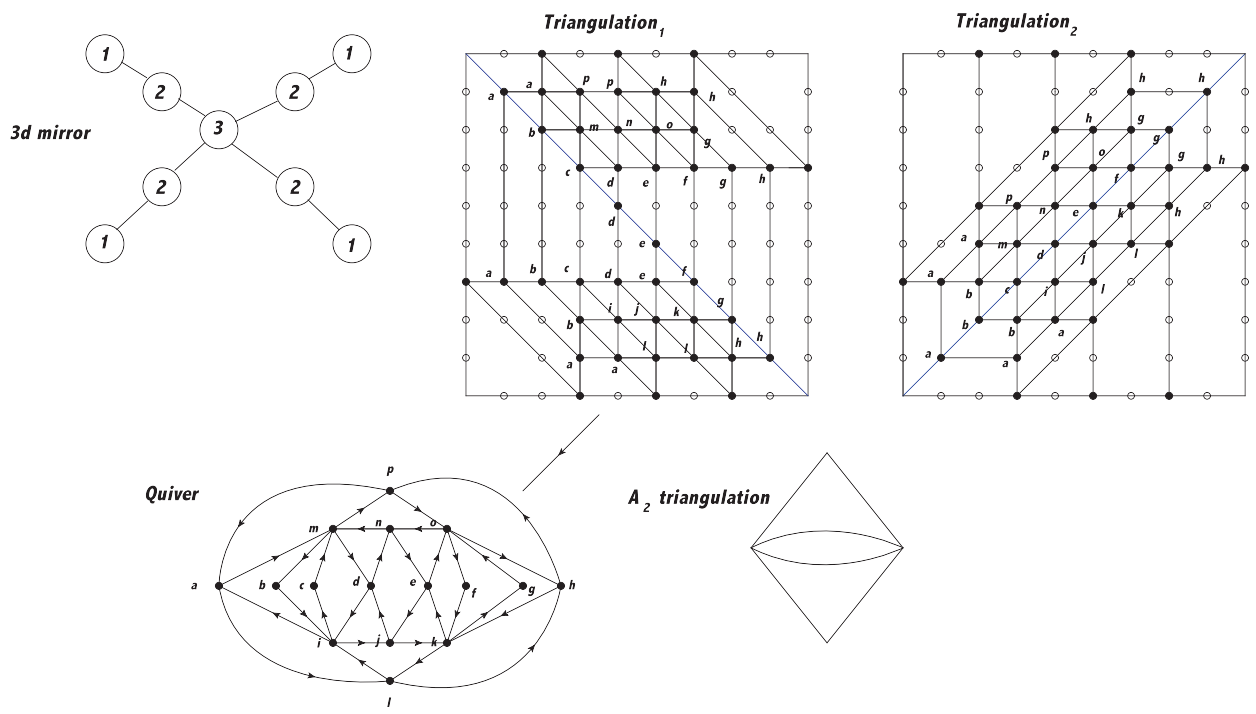

Figure 58: The quiver for the theory defined by a $A_{2}$ theory compactified on a sphere with four full punctures.

For general $N$, based on the assumption that the number of BPS states in minimal chamber is a polynomial function of $N$ and the scaling behavior is $N^{3}$, we conjecture that the function is

$$
f(N)=2 N(N-1)(2 N-1)
$$

Using this method, one can find finite chambers for any theory defined on a sphere with regular punctures. 


\section{Spectral generator}

Let's first review the meaning of the spectral generator of $A_{1}$ case introduced in [13], and we will rephrase it in terms of the language of cluster algebra. The Seiberg-Witten curve of the theory is

$$
x^{2}=\Phi(z),
$$

where $z$ is the coordinate on the Riemann surface, $x$ is the coordinate on the cotangent bundle, and $\phi(z)$ is a quadratic differential defined on the Riemann surface. The Seiberg-Witten differential is $\lambda=x d z=\sqrt{\Phi(z)} d z$ and one can use it to define a foliation on the Riemann surface from the following flow equation

$$
\lambda \frac{d z}{d t}=e^{i \theta}
$$

here $\theta$ is a fixed angle and in fact is the slop of the BPS hypermultiplet for some critical value $\theta$. For more details on the structure of the foliation, please see $[13,69,70]$. What we want to point out is that the topology of the foliation is exactly equivalent to the bipartite network introduced earlier and therefore also equivalent to the triangulation, see [71]. The branch points of the Seiberg-Witten differential is the vertex for the foliation which is identified with the black vertices of our network, see Figure 59.

When $\theta$ is changed, the foliation is also changed smoothly. However, when $\theta$ arrives at a critical value, then the topology of the foliation is changed due to the appearance of a hypermultiplet which is represented by the flow lines connected by two branch points. Locally, this change of topology is just the square move for the network which then corresponds to the quiver mutations. The $\theta$ actually parameterizes one half plane of the central charge, therefore by rotating $\theta$ angle 180 degree, one can probe all the BPS particles by tracking the change of the topology of the network. This is essentially the same as finding a maximal green mutation of the original quiver (for the chamber with finite number of states).

Now for each face of the network, one can associate a cluster $X$ coordinate which parameterizes the framed moduli space of flat connections defined on the Riemann surface. The appearance of the hypermultiplet acts like a square move on the network and therefore acts like a cluster transformation on the coordinate. When the $\theta$ angle is rotated by 180 degrees, the cluster coordinates for each face are changed to a certain value. Moreover, 


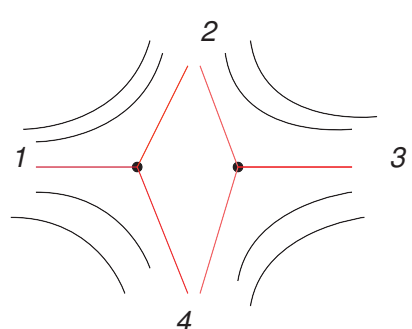

flow lines

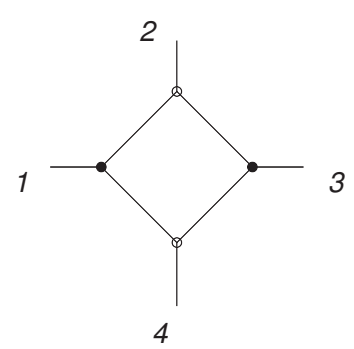

bipartite network

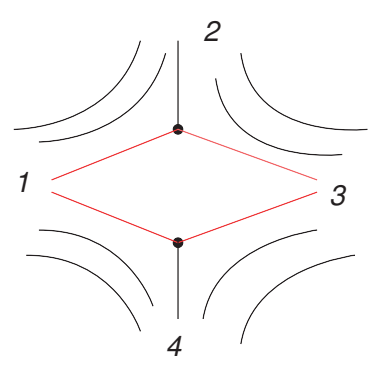

flow lines after the appearance of a hypermultiplet

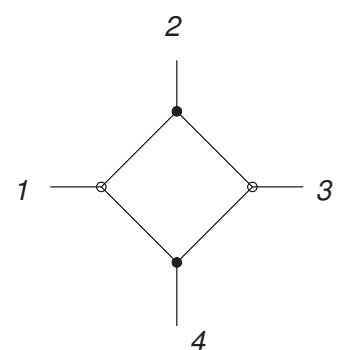

New network which is the actually the square move

Figure 59: Top: The local picture of the flow lines and the equivalent bipartite network. Bottom: The new flow line after an appearance of the hypermulitplet and the new bipartite network, which is actually the square move of the original network.

the final cluster coordinates do not depend on the chamber one probe as long as long as initial foliation is given.

However, it is usually difficult to track the change of the network and therefore hard to find the BPS spectrum. There is an easy way of getting the final cluster coordinate without knowing the detailed spectrum information. The method uses the definition of the cluster coordinates from the cross ratio of the flags [33] attached on each marked points. The rotation by 180 degree acts like a $Z_{2}$ action on the attached flag, and the change of the cluster coordinates can be worked out explicitly using the definition of the cross ratio. The final cluster coordinates is the spectral generator from which one could find sensible factorization and therefore the explicit spectrum information.

In this paper, we are not pursing a similar geometric derivation of the spectral generator for the higher rank theory, which we will discuss in another occasion. However, we will give a derivation using the information of 
the BPS spectrum though, in particular, the maximal green mutation is very useful.

One of the remarkable feature of the maximal green mutation is that it keeps track of the position of the quiver nodes. Let's label the frozen nodes as $(1,2, \ldots, n)$ which is the same labeling of the original quiver. Then after doing the maximal green mutations, the cluster $X$ coordinate for $n$th quiver node is equal to the coordinate of the quiver nodes connected to the $n$th frozen node. Let's check this explicitly using the simple pentagon geometry of the $A_{1}$ theory. The two chambers and the final cluster coordinates are shown in Figure 60. The final coordinates from chamber 1 is

$$
X_{1}^{f}=X_{1}^{\prime}=x_{1}^{-1}\left(1+x_{2}+x_{2} x_{1}\right), \quad X_{2}^{f}=X_{2}^{\prime}=x_{2}^{-1}\left(1+x_{1}\right)^{-1} .
$$

and the results are the same from chamber 2 due to the permutation of the quiver nodes! This expression is clearly the same as the one given in [13], see formula 11.27 and 11.28 (with a slightly different convention for the definition of the coordinate.).
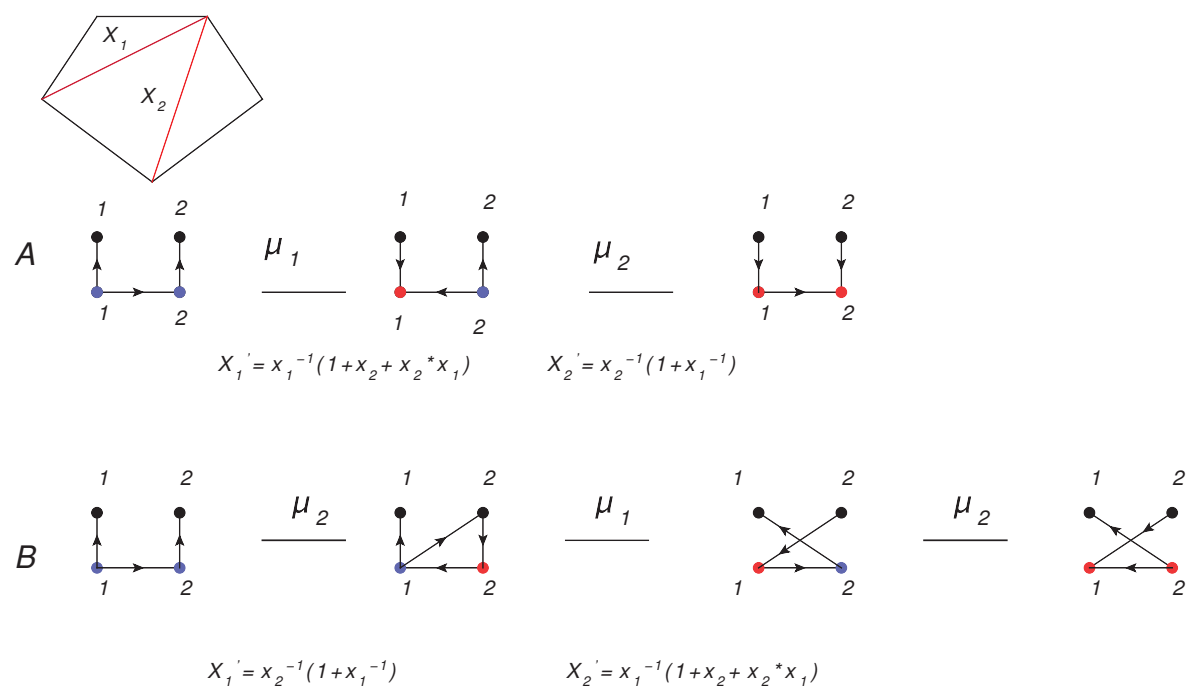

Figure 60: Top: The triangulation for the five punctured disc which gives the BPS quiver for $A_{2} \mathrm{AD}$ theory. Bottom: Two BPS chambers from the maximal green mutations and the final cluster coordinates.

Exact similar consideration can be generalized to higher rank theories: the spectral generator can be written down using the explicit maximal green mutation sequences. Let's consider a disc with four full punctures, and the 
initial configuration and the final configuration of the maximal green mutation is listed in Figure 61. The mutation sequences are

$$
\begin{aligned}
& (1,5,9),(2,4,6,8),(3,5,7), \\
& (1,2,4,1),(9,6,8,9),
\end{aligned}
$$

and the final coordinates or the spectral generator can be written down using the mutation formula for the $X$ coordinate and the permutation:

$$
\begin{aligned}
& X_{1}^{f}=X_{3}^{\prime}, X_{2}^{f}=X_{2}^{\prime}, X_{3}^{f}=X_{1}^{\prime}, \\
& X_{4}^{f}=X_{6}^{\prime}, X_{5}^{f}=X_{5}^{\prime}, X_{6}^{f}=X_{4}^{\prime}, \\
& X_{7}^{f}=X_{9}^{\prime}, X_{8}^{f}=X_{8}^{\prime}, X_{6}^{f}=X_{9}^{\prime} .
\end{aligned}
$$
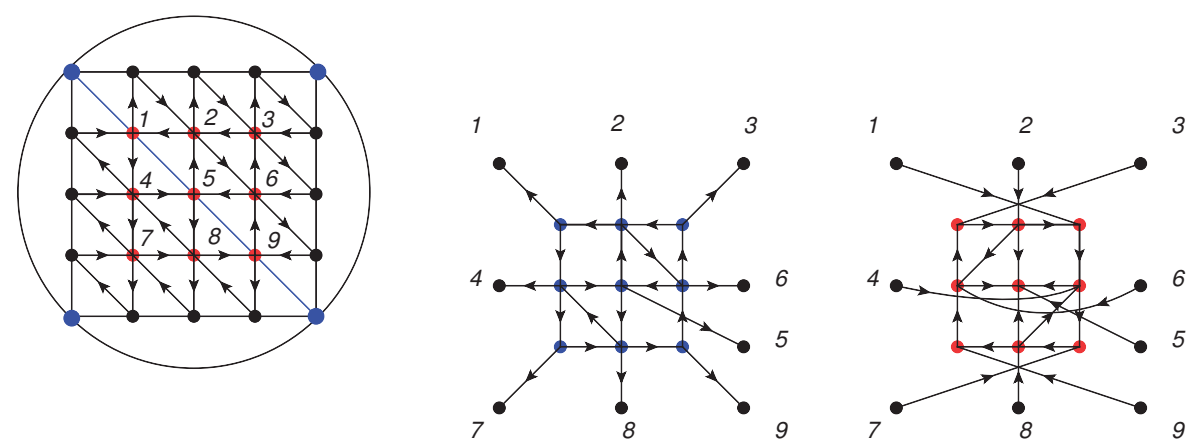

Figure 61: The initial configuration and the final configuration of the maximal green mutation of the quiver from the disc with four full punctures.

The expression is rather long and the interested reader can find the expression in the appendix A. One can write down the spectral generator for all the theories whose BPS spectrum is discovered in this paper, many examples are given in the appendix A. It would be interesting to find other factorizations of the spectral generator which will give the BPS spectrum in other chamber.

Since there is a quantum cluster algebra, then one could also define a refined version of the spectral generator, which would then tell us the spin information of the BPS particles. The refined spectral generator can be easily found using the explicit mutation sequences, let's consider the example 
shown in Figure 60, the noncommutative commutation relation is

$$
X_{\alpha} X_{\beta}=q^{\epsilon_{\alpha \beta}} X_{\beta} X_{\alpha}
$$

and the quantum cluster transformation is

$$
\begin{aligned}
& X_{k}^{\prime}=X_{k}^{-1} \\
& X_{i}^{\prime}=X_{i}\left(\prod_{a=1}^{\left|\epsilon_{i k}\right|}\left(1+q^{a-1 / 2} X_{k}^{-\operatorname{sgn}\left(\epsilon_{i k}\right)}\right)\right)^{-\operatorname{sgn}\left(\epsilon_{i k}\right)}
\end{aligned}
$$

Using the above quantum cluster transformation, the refined spectral generator can be written down using the mutation sequences from chamber 1

$$
X_{1}^{f}=X_{1}^{-1}+q^{\frac{1}{2}} X_{1}^{-1} X_{2}+X_{2}, \quad X_{2}^{f}=\frac{1}{X_{2}+q^{-\frac{1}{2}} X_{2} X_{1}}
$$

In the limit $q \rightarrow 1$, the refined spectral generator is the same as the classical one given in [174]. A more complicated example of refined spectral generator is given in appendix $\mathrm{B}$.

\section{Vector multiplets}

\subsection{Wall crossing between chamber with Infinite number of states}

Previous sections focused on the finite chambers and their wall crossing behavior. In this section, we would like to say something about the chamber with higher spin states using the quiver representation theory. Our treatment is very elementary and we hope to do a more thorough analysis in the future.

There are also wall crossing behavior between two chambers with infinite states, as discovered in original paper of Seiberg-Witten [3]. We can actually see this type of wall crossing behavior using the quiver representation theory for some simple examples. Consider $S U(2)$ theory with one flavor whose BPS quiver is of the type $\tilde{A}(2,1)$ shown in Figure 62 , the possible BPS states are represented by the curves on the triangulated Riemann surface, which are listed in Table 13. 

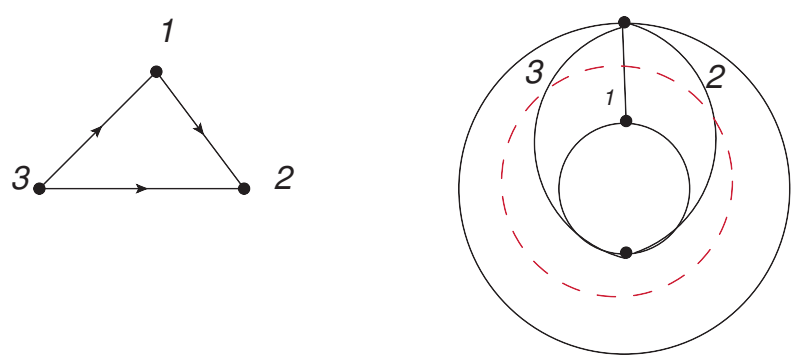

Figure 62: The triangulation and BPS quiver for $S U(2)$ theory with one flavor, and the curve corresponding to the $W$ boson is drawn as the red curve.

\begin{tabular}{|c|c|}
\hline & charge \\
\hline Hypermultiplet & $\left(\gamma_{i}+\gamma_{j}, i \neq j\right),\left(n \gamma_{i}+n \gamma_{j}+(n \pm 1) \gamma_{k}, i \neq j \neq k\right)$ \\
\hline Vector multiplet & $\gamma_{1}+\gamma_{2}+\gamma_{3}$ \\
\hline
\end{tabular}

Table 13: The indecomposable representation of the quiver $\tilde{A}(2,1)$.

\begin{tabular}{|c|c|c|}
\hline Name & Indecomposable & subrepresentation \\
\hline $\mathrm{A}$ & $\gamma_{1}+\gamma_{2}+\gamma_{3}$ & $B, S_{2}$ \\
\hline $\mathrm{B}$ & $\gamma_{1}+\gamma_{2}$ & $S_{2}$ \\
\hline $\mathrm{C}$ & $\gamma_{1}+\gamma_{3}$ & $S_{1}$ \\
\hline $\mathrm{D}$ & $\gamma_{2}+\gamma_{3}$ & $S_{2}$ \\
\hline$E_{n}$ & $n \gamma_{1}+n \gamma_{2}+(n+1) \gamma_{3}$ & $A, B, S_{2}, D$ \\
\hline$F_{n}$ & $n \gamma_{1}+n \gamma_{2}+(n-1) \gamma_{3}$ & $A, B, S_{2}$ \\
\hline$G_{n}$ & $n \gamma_{1}+n \gamma_{3}+(n+1) \gamma_{2}$ & $B, S_{2}$ \\
\hline$H_{n}$ & $n \gamma_{1}+n \gamma_{3}+(n-1) \gamma_{2}$ & $A, B, S_{2}$ \\
\hline$I_{n}$ & $n \gamma_{2}+n \gamma_{3}+(n+1) \gamma_{1}$ & $A, B, S_{2}$ \\
\hline$J_{n}$ & $n \gamma_{2}+n \gamma_{3}+(n-1) \gamma_{1}$ & $A, B, S_{2}, D$ \\
\hline
\end{tabular}

Table 14: The subrepresentations of the indecomposable representation of the quiver $\tilde{A}(2,1)$.

Representation $A$ is the $W$ boson whose subrepresentations are $\gamma_{2}, \gamma_{1}+$ $\gamma_{2}{ }^{28}$, so it is stable if the slop of its subrepresentations are smaller than $\gamma_{2}$

${ }^{28}$ Let's give a little bit explanation of the sub-representation listed in Table For example, $D$ is a subrepresentation of series $E_{1}$ because one can select a one dimensional subspace of $V_{3}=2$ such that it maps to zero of $V_{1}=1$, etc, and therefore the representation $D=(0,1,1)$ is a subrepresentation of $E_{1}$. 
and $\gamma_{1}+\gamma_{2}$. There are two choices of the stability conditions on the simple representations which will make the $W$ boson stable, one of them is shown in Figure 63 (another is given by exchanging $S_{1}$ and $S_{2}$.). There are more choices depending on the relative slop of other representations. Let's explain this using the results shown in Figure 63. f In chamber one, representations $F_{n}$ and $I_{n}$ are unstable since their subrepresentation $A$ is on their right. The reason is the following: consider representations $F_{n}$ whose charge vector can be decomposed as:

$$
\begin{aligned}
n \gamma_{1}+n \gamma_{2}+(n-1) \gamma_{3} & =(n-1)\left(\gamma_{1}+\gamma_{2}+\gamma_{3}\right)+\gamma_{1}+\gamma_{2} \\
& =(n-1) A+B
\end{aligned}
$$

so $F_{n}$ is lying in between $A$ and $B$, and $A$ as its subrepresentation is on its right, so it is unstable! Similarly $J_{n}$ is also unstable since its subrepresentation $D$ is always on its right. $C, D, G_{n}, H_{n}$ are always stable since all their proper sub representations have higher slops. Finally the stability of $E_{n}$ depends on on the relative position of $C$ and $D$, since $E_{n}$ series lie in between the charge vector $C$ and $A$, all $E_{n}$ are unstable if $D$ is on the right of $C$ as shown in chamber 1 of Figure 63 , On the other hand, if $D$ is on the left of $C$, then some of the representations of $E_{n}$ series are lying in between $C$ and $D$ and they are stable, the truncation of the $E_{n}$ series depend on the relative position of $C$ and $D$, so we have an infinite number of chambers!

If the relative order of $S_{1}$ and $S_{2}$ are interchanged, then $B=\gamma_{1}+\gamma_{2}$ are stable and the analysis of other stable particles are the same, the results are shown in Table 15.

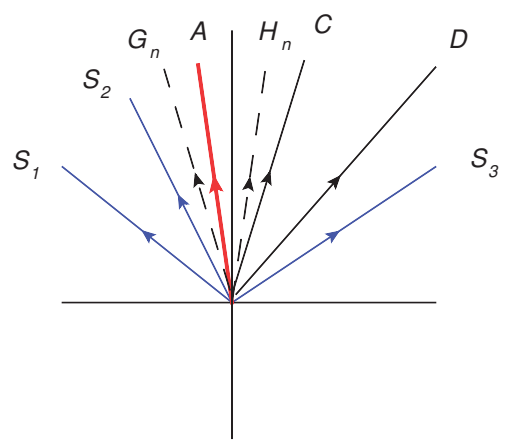

Chamber $_{1}$

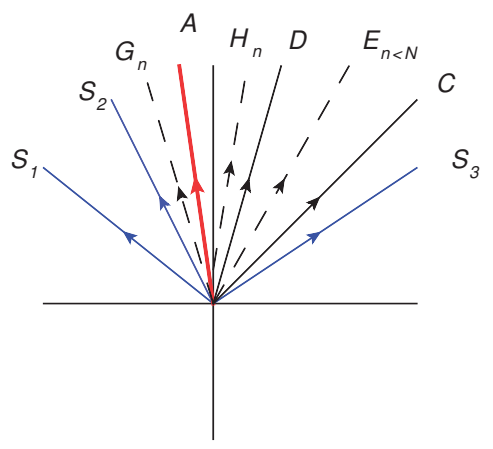

Chamber $_{2}$

Figure 63: Two choices of stability condition which will make $W$ boson stable. 


\begin{tabular}{|c|c|}
\hline & Stable particles \\
\hline Chamber 1 & $S_{1}, S_{2}, G_{n}, A, H_{n}, C, D, S_{3}$ \\
\hline Chamber 2 & $S_{1}, S_{2}, G_{n}, A, H_{n}, D, E_{n<N}, C, S_{3}$ \\
\hline Chamber 3 & $S_{2}, B, S_{1} G_{n}, A, H_{n}, C, D, S_{3}$ \\
\hline Chamber 2 & $S_{2}, B S_{1}, G_{n}, A, H_{n}, D, E_{n<N}, C, S_{3}$ \\
\hline
\end{tabular}

Table 15: The BPS spectrum with infinite number of states of $S U(2)$ with one flavor.

The above infinite chambers can be found using the green mutation and red mutation method. Let's consider chamber 1, we can do the green mutations

$$
\mu_{1}, \mu_{2}, \mu_{3}, \mu_{2}, \mu_{3}, \mu_{2} \ldots
$$

The charge vectors for the infinite mutation sequences are exactly the states on the left of the $W$ boson:

$$
S_{1}, S_{2}, G_{n}
$$

see the top of Figure 64. The green mutation only probes this part of the spectrum. To probe the other part of the spectrum, one need to use the red mutations. Since $\gamma_{1}$ and $\gamma_{2}$ are already probed, one can only start mutating node 3 ; After first step, one can mutate either node 2 or node 1 , we choose to mutate node 2 . In third step, there are still two choices: either mutate node 3 or node 1 , however, the charge vector for node 3 is $\gamma_{1}$ which has already found by doing green mutation, therefore we can only mutate node 1 in this step, there is no ambiguity in the later mutations, the red mutations are

$$
\mu_{3}, \mu_{2}, \mu_{1}, \mu_{3}, \mu_{1}, \mu_{3}, \mu_{1}, \ldots
$$

and the charge vectors for this part are

$$
S_{3}, \quad D, \quad C, \quad H_{n}
$$

Combining the green mutation and red mutation, we find $W$ boson as the common limit and recover the BPS states in chamber one. It is very easy to recover the other chambers using the mutation method. 

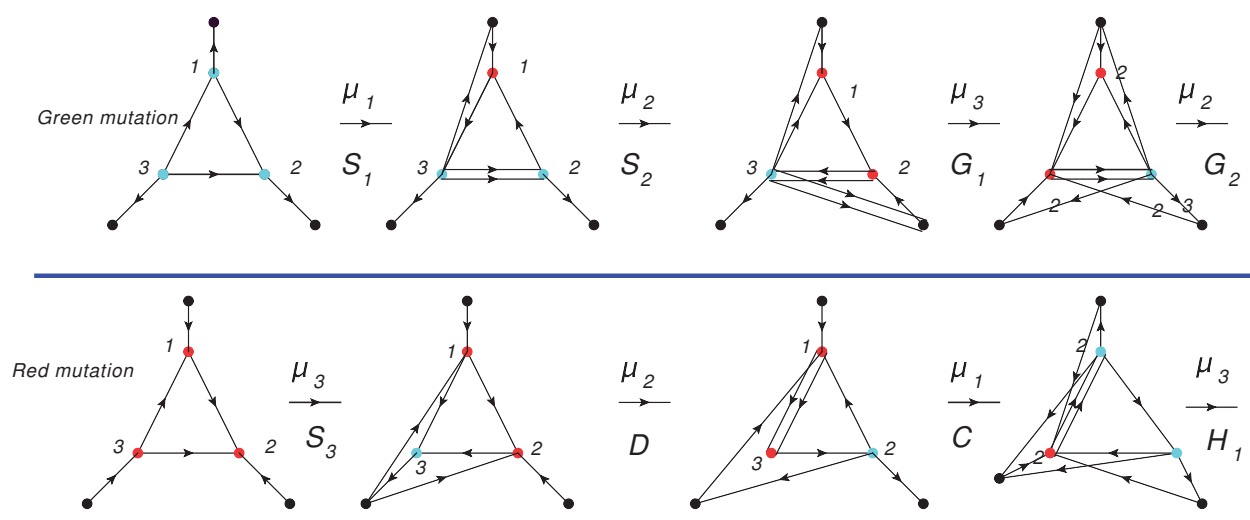

Figure 64: Top: The green mutation with infinite number of steps. Bottom: The red mutation with infinite number of steps which approach to the same limit as the green mutation at the top.

\subsection{Theory without finite chamber}

The $S U(2) N=2^{*}$ theory has no finite chamber [21]. Here let's give a very simple proof using the quiver representation theory. The $\operatorname{BPS}(Q, W)$ for this theory is shown in Figure 65. The three $W$ boson are represented by the subquiver of pure $S U(2)$ theory, and each node is a sink for the $W$ boson, so if we start mutating any of the quiver nodes, one of the $W$ boson would be stable, and there is no way we can find a finite chamber.

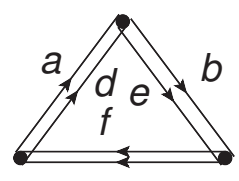

C

\section{$W=a b c+d e f+a b c d e f$}

Figure 65: The BPS quiver for $S U(2)$ with one massive adjoint, the superpotential is also given.

It is easy to generalize the above analysis to $\mathcal{N}=2^{*} S U(N)$ theory. The BPS quiver for $S U(3)$ with one massive adjoint is given in [34]. The triangulation, dot diagram and the quiver is shown in Figure 66 for $N=3$.

The subquivers representing the $W$ bosons are listed in Table 16. Let's now look at the green mutation sequences. Because of the existence of the 

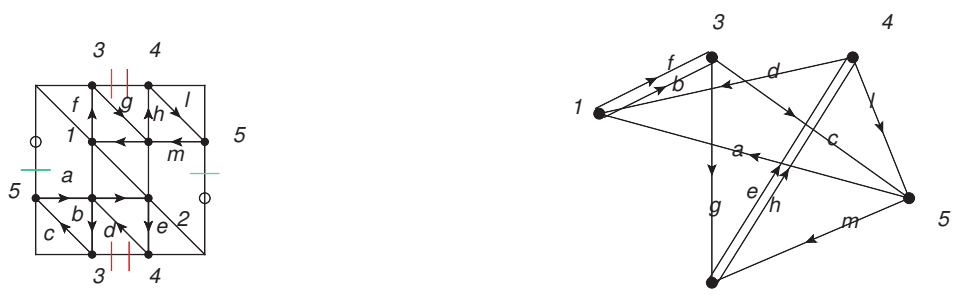

2

$W=a b c+d f g e+\mid h m+a f c m e l+g h d b$

Figure 66: The BPS quiver for $S U(3)$ with one massive adjoint, and the superpotential is also given.

vector boson represented by $W 1$ and $W 2$, one could not mutate node 3 and 4 in first step. If we mutate node 2 and 1 in first step, then in second step we can only mutate nodes 3 and 4 , since mutating node 5 would make $W 3$ and $W 4$ stable. Similarly, one can only mutate nodes 1 and 2 in third step to avoid activating $W 3$ or $W 4$, but this will finally make $W 5$ stable! Therefore there is no finite chamber for $S U(3)$ with a massive adjoint. Similar analysis can be done to the general $S U(N)$ theory and the conclusion is that there is no finite chamber for $S U(N)$ theory with a massive adjoint!

\begin{tabular}{|c|c|c|c|}
\hline Name & Quiver nodes & Source & Sink \\
\hline W1 & $(1,3)$ & 1 & 3 \\
\hline W2 & $(2,4)$ & 2 & 4 \\
\hline W3 & $(2,3,5)$ & 3 & 2 \\
\hline W4 & $(1,4,5)$ & 4 & 1 \\
\hline W5 & $(1,3,5,4)$ & 4 & 5 \\
\hline
\end{tabular}

Table 16: The subquiver of vector bosons and the source-sink analysis for the BPS quiver of SU(3) with one adjoint.

\section{Conclusion}

Finite BPS chambers are found for a large class of $4 \mathrm{~d} \mathcal{N}=2$ theories engineered from six dimensional $A_{N-1}(2,0)$ theory on a Riemann surface with regular and irregular singularity. Our results greatly extend the knowledge of the BPS spectrum of various kinds of higher rank $\mathcal{N}=2$ quantum field 
theories, and should be a first step towards a full understanding of the BPS spectrum of these theories. There are many open questions which deserve further study:

a. It is interesting to find all the finite chambers for a given BPS quiver of a given theory. Since an explicit combinatorial algorithm is given, it may be possible to do it using computer scanning. It is also interesting to do the similar scanning for all the BPS quivers of a given theory, and try to answer the following questions: what is the minimal chamber and what is the maximal chamber? whether any length between the minimal and maximal length is realized as the length of a BPS chamber, etc.

b. In fact, the spectrum we found should be called potential chamber of the theory, and it would be interesting to see if such spectrum is truly realized on the Coulomb branch. It seems to us that all the finite chamber can be realized on the moduli space, but this definitely needs further study. Although the detailed factorization might not be realized physically, the spectral generator is the correct one regardless of the chamber, and it is interesting to explore how to find the sensible factorizations of the given spectral generator and therefore find new chambers.

c. We have not found any efficient way to deal with chamber with higher spin states. It would be nice to find new methods to deal with this problem.

$\mathrm{d}$. The BPS counting in the supergravity context is studied in [51, 72-80], can we apply our combinatorial method to that context?

With the BPS spectrum on hand, there are many physical questions one could ask:

a. Why the spectrum of a given theory has the specific structure? Can we learn about the UV theory from the BPS data? Recently, it is proposed that the BPS spectrum can be used to calculate the index of the superconformal field theory [81], it would be interesting to carry out this explicitly. Furthermore, the finite spectrum usually happens in the strongly coupled region of the Lagrangian theory, and the massless BPS particles at the singularity of the Coulomb branch should be included into the stable BPS spectrum. Since the BPS particles at the singularity is very important in understanding the IR physics, can we learn something deep about the quantum dynamics like the exact solutions and confinement from the explicit BPS spectrum?

b. The quantum dilogarithm identify has important implications for mirror symmetry of $3 \mathrm{~d} \mathcal{N}=2$ theory [82, 83]. We have found a huge number of new quantum dilogarithm identities, can we use them to find new $3 \mathrm{~d}$ Mirror pairs? The quantum dilogarithm identity is closed related to the integrable system [84], it would be interesting to find the detailed connection. 
c: The finite spectrum has an interesting $N^{3}$ scaling behavior in the large $\mathrm{N}$ limit, which is in agreement with the degree of freedom of $N$ M5 branes. Since the BPS states can be thought of as self-dual string wrapping on various one cycles on the punctured Riemann surface, it is natural to think this $N^{3}$ scaling should be related to M5 brane dynamics.

d. The BPS spectrum is important for finding the Coulomb branch metric of the corresponding $3 \mathrm{~d}$ theory derived by compactifying $4 \mathrm{~d}$ theory on a circle, and it would be interesting to find out the Hyperkahler metric explicitly this using the BPS spectrum [85].

Acknowledgments. We thank M. Alim, M. Del Zotto, D. Gaiotto, A. Neitzke, C. Vafa and M. Yamazaki for help discussions. We thank Yutin Huang for help on a mathematica code. This research is supported in part by Zurich Financial services membership and by the U.S. Department of Energy, grant DE- FG02-90ER40542 (DX). We thank the 10th Simons Summer Workshop in Mathematics and Physics, and the Simons Center for Geometry and Physics for hospitality where part of this work is finished.

\section{Appendix A. Explicit spectral generator}

\section{A.1. $A_{3}$ quiver}

In this section, we give some explicit expression for the spectral generator using the cluster transformation rule for the $X$ coordinates under mutation on node $k$,

$$
\begin{aligned}
& X_{k}^{\prime}=X_{k}^{-1} \\
& X_{i}^{\prime}=X_{i}\left(1+X_{k}^{-\operatorname{sgn}\left(\epsilon_{i k}\right)}\right)^{-\epsilon_{i k}} .
\end{aligned}
$$

For the $A_{3}$ quiver shown in Figure A1A, the maximal green mutation sequences are

$$
\mu_{1}, \mu_{2}, \mu_{3}, \mu_{1}
$$

The final cluster coordinates are: ${ }^{29}$

\footnotetext{
${ }^{29}$ We only show the cluster coordinates due to the mutation, the permutation can be found using the green mutation as we show in Figure A1A, the spectral generator is derived by combining the permutation and the cluster transformation.
} 
$\hat{x}[1]=\frac{1+x_{1}+x_{1} x_{2}}{x_{2}\left(1+x_{3}+x_{1} x_{3}\right)}$,
$\hat{x}[2]=\frac{1+x_{3}+x_{1} x_{3}}{x_{1}\left(1+x_{2}+x_{2} x_{3}\right)}$,
$\hat{x}[3]=\frac{1+x_{2}+x_{2} x_{3}}{\left(1+x_{1}+x_{1} x_{2}\right) x_{3}}$
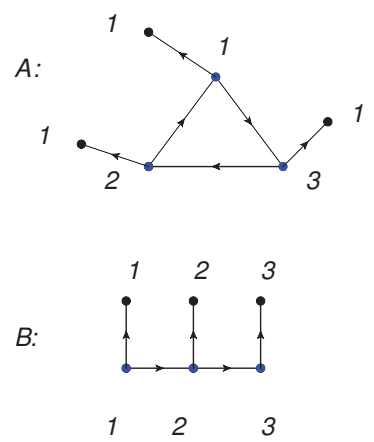
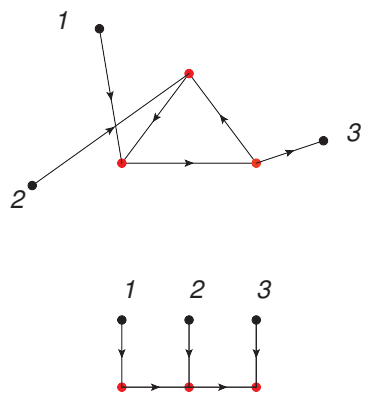

Figure A1: Two quivers in $A_{3}$ mutation class, we show the initial configuration and final configuration of the maximal green mutation.

If we use another quiver in the same mutation class as the above quiver, say the quiver in Figure A1B, then one maximal green mutation sequences are

$$
\mu_{1}, \mu_{2}, \mu_{3}
$$

and the final cluster coordinates would be:

$\hat{X}[1]=\frac{1+x_{2}+x_{1} x_{2}}{x_{1}}$,

$\hat{X}[2]=\frac{1+x_{3}+x_{2} x_{3}+x_{1} x_{2} x_{3}}{\left(1+x_{1}\right) x_{2}}$

$\hat{X}[3]=\frac{1}{\left(1+x_{2}+x_{1} x_{2}\right) x_{3}}$.

Notice that the final coordinates depend on the initial quiver, but the quantum dilogarithm identity is independent of the quiver, they are all equal by changing the basis of the quantum torus from one quiver to another quiver.

\section{A.2. Disc with three $A_{N-1}$ full punctures}

Let's consider a disc with three full punctures and $N=5$, the BPS quiver is shown in Figure A2, and the maximal mutation sequence is

$$
\mu_{1}, \mu_{2}, \mu_{3}, \mu_{4}, \mu_{5}, \mu_{6}, \mu_{1}, \mu_{2}, \mu_{3}, \mu_{1}
$$


We find the spectral generator (final cluster coordinates) as following

$\hat{X}[1]=\frac{1+x_{3}+x_{2} x_{3}+x_{3} x_{6}+x_{2} x_{3} x_{6}+x_{2} x_{3} x_{5} x_{6}}{\left(1+x_{5}+x_{3} x_{5}+x_{4} x_{5}+x_{3} x_{4} x_{5}+x_{2} x_{3} x_{4} x_{5}\right) x_{6}}$

$\hat{X}[2]=\frac{\left(1+x_{2}+x_{1} x_{2}+x_{2} x_{5}+x_{1} x_{2} x_{5}+x_{1} x_{2} x_{3} x_{5}\right)\left(1+x_{6}+x_{5} x_{6}+x_{4} x_{5} x_{6}\right)}{\left(1+x_{4}+x_{2} x_{4}+x_{1} x_{2} x_{4}\right) x_{5}\left(1+x_{3}+x_{2} x_{3}+x_{3} x_{6}+x_{2} x_{3} x_{6}+x_{2} x_{3} x_{5} x_{6}\right)}$,

$\hat{X}[3]=\frac{\left(1+x_{5}+x_{3} x_{5}+x_{4} x_{5}+x_{3} x_{4} x_{5}+x_{2} x_{3} x_{4} x_{5}\right)\left(1+x_{1}+x_{1} x_{3}+x_{1} x_{3} x_{6}\right)}{x_{3}\left(1+x_{2}+x_{1} x_{2}+x_{2} x_{5}+x_{1} x_{2} x_{5}+x_{1} x_{2} x_{3} x_{5}\right)\left(1+x_{6}+x_{5} x_{6}+x_{4} x_{5} x_{6}\right)}$,

$\hat{X}[4]=\frac{1+x_{5}+x_{3} x_{5}+x_{4} x_{5}+x_{3} x_{4} x_{5}+x_{2} x_{3} x_{4} x_{5}}{x_{4}\left(1+x_{2}+x_{1} x_{2}+x_{2} x_{5}+x_{1} x_{2} x_{5}+x_{1} x_{2} x_{3} x_{5}\right)}$

$\hat{X}[5]=\frac{\left(1+x_{4}+x_{2} x_{4}+x_{1} x_{2} x_{4}\right)\left(1+x_{3}+x_{2} x_{3}+x_{3} x_{6}+x_{2} x_{3} x_{6}+x_{2} x_{3} x_{5} x_{6}\right)}{x_{2}\left(1+x_{5}+x_{3} x_{5}+x_{4} x_{5}+x_{3} x_{4} x_{5}+x_{2} x_{3} x_{4} x_{5}\right)\left(1+x_{1}+x_{1} x_{3}+x_{1} x_{3} x_{6}\right)}$,

$\hat{X}[6]=\frac{1+x_{2}+x_{1} x_{2}+x_{2} x_{5}+x_{1} x_{2} x_{5}+x_{1} x_{2} x_{3} x_{5}}{x_{1}\left(1+x_{3}+x_{2} x_{3}+x_{3} x_{6}+x_{2} x_{3} x_{6}+x_{2} x_{3} x_{5} x_{6}\right)}$.

Here $x_{i}$ is the initial cluster variable. This result is the same as the formula $[7.13-7.15]$ in [23] if we identify the initial coordinates in the following way

(A.5) $x_{1}=r_{200}, \quad x_{2}=r_{110} x_{3}=r_{011}, \quad x_{4}=r_{020}, \quad x_{5}=r_{101}, \quad x_{6}=r_{002}$.
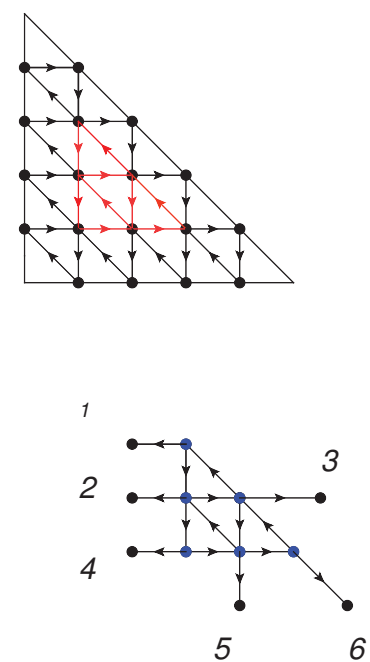
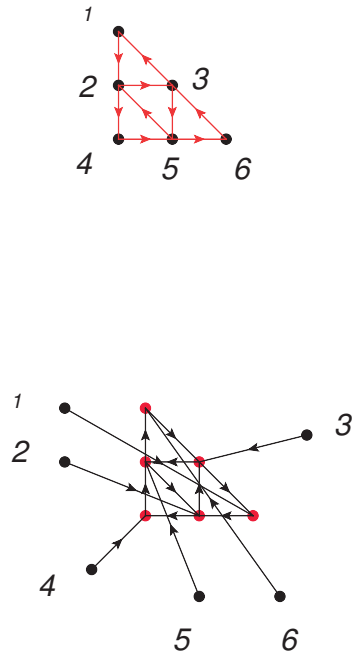

Figure A2: The quiver from disc with three full punctures and $N=5$. The initial and final configuration of the maximal green mutation are indicated. 


\section{A.3. Disc with four $A_{N-1}$ full punctures}

The quiver is shown in Figure A3, and the green mutation sequence is

$$
\begin{aligned}
& (1,5,9),(2,4,6,8),(3,5,7), \\
& (1,2,4,1),(9,6,8,9),
\end{aligned}
$$
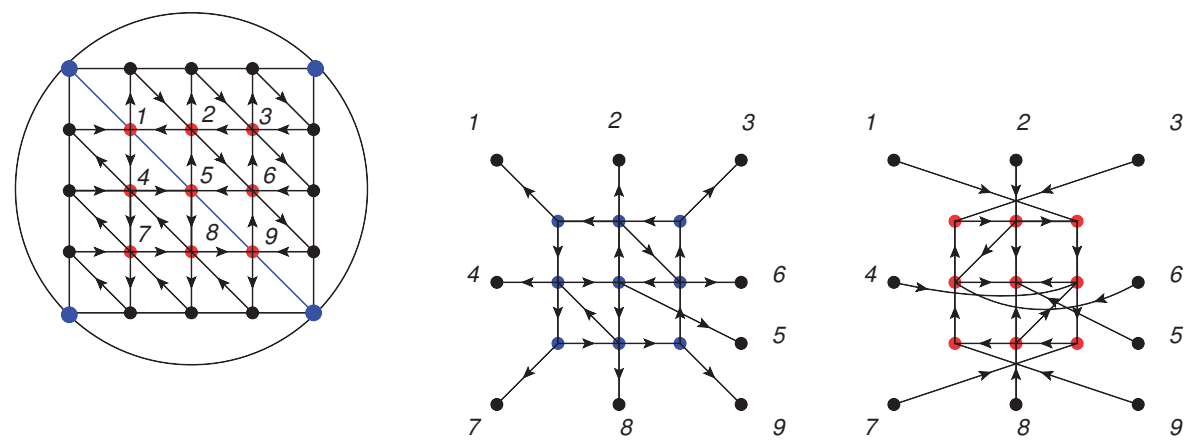

Figure A3: The initial configuration and the final configuration of the maximal green mutation of the quiver from the disc with four full punctures.

We take $N=4$ and the spectral generator is

$$
\begin{aligned}
& \hat{X}[1]=\frac{1+x_{2}+x_{2} x_{3}+x_{2} x_{5}+x_{2} x_{3} x_{5}+x_{2} x_{4} x_{5}+x_{2} x_{3} x_{4} x_{5}+x_{2} x_{3} x_{5} x_{6}+x_{2} x_{3} x_{6} x_{5} x_{6}+x_{2} x_{3} x_{5} x_{6} x_{9}+x_{2} x_{3} x_{6} x_{5} x_{6} x_{9}+x_{2} x_{3} x_{4} x_{5} x_{6} x_{8} x_{9}}{x_{3}\left(1+x_{6}+x_{2} x_{6}+x_{6} x_{9}+x_{2} x_{6} x_{9}+x_{6} x_{8} x_{9}+x_{2} x_{6} x_{8} x_{9}+x_{2} x_{5} x_{6} x_{8} x_{9}+x_{6} x_{7} x_{8} x_{9}+x_{2} x_{6} x_{7} x_{8} x_{9}+x_{2} x_{5} x_{6} x_{7} x_{8} x_{9}+x_{2} x_{4} x_{5} x_{6} x_{7} x_{8} x_{9}\right)},
\end{aligned}
$$

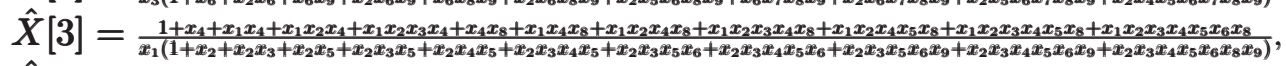

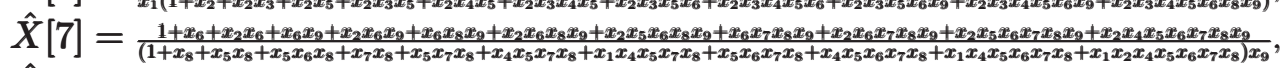

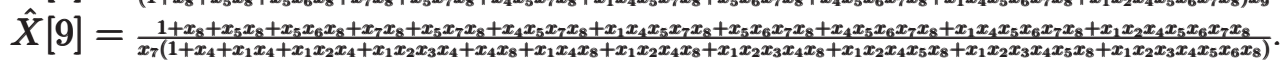

$$
\begin{aligned}
& \hat{X}[2]=\frac{\mathrm{A} 2 * \mathrm{~B} 2}{\mathrm{C} 2 * \mathrm{D} 2} \\
& \mathrm{~A} 2=\left(1+x_{1}+x_{1} x_{2}+x_{1} x_{2} x_{3}+x_{1} x_{2} x_{5}+x_{1} x_{2} x_{3} x_{5}+x_{1} x_{2} x_{3} x_{5} x_{6}\right. \\
& \left.+x_{1} x_{2} x_{3} x_{5} x_{6} x_{9}\right) \text {, } \\
& \mathrm{B} 2=\left(1+x_{6}+x_{2} x_{6}+x_{6} x_{9}+x_{2} x_{6} x_{9}+x_{6} x_{8} x_{9}+x_{2} x_{6} x_{8} x_{9}+x_{2} x_{5} x_{6} x_{8} x_{9}\right. \\
& \left.+x_{6} x_{7} x_{8} x_{9}+x_{2} x_{6} x_{7} x_{8} x_{9}+x_{2} x_{5} x_{6} x_{7} x_{8} x_{9}+x_{2} x_{4} x_{5} x_{6} x_{7} x_{8} x_{9}\right) \text {, } \\
& \mathrm{C} 2=\left(1+x_{5}+x_{4} x_{5}+x_{1} x_{4} x_{5}+x_{5} x_{6}+x_{4} x_{5} x_{6}+x_{1} x_{4} x_{5} x_{6}+x_{1} x_{2} x_{4} x_{5} x_{6}\right. \\
& +x_{5} x_{6} x_{9}+x_{4} x_{5} x_{6} x_{9}+x_{1} x_{4} x_{5} x_{6} x_{9}+x_{1} x_{2} x_{4} x_{5} x_{6} x_{9}+x_{4} x_{5} x_{6} x_{8} x_{9} \\
& \left.+x_{1} x_{4} x_{5} x_{6} x_{8} x_{9}+x_{1} x_{2} x_{4} x_{5} x_{6} x_{8} x_{9}+x_{1} x_{2} x_{4} x_{5}^{2} x_{6} x_{8} x_{9}\right) \text {, } \\
& \mathrm{D} 2=x_{2}\left(1+x_{3}+x_{3} x_{6}+x_{3} x_{6} x_{9}+x_{3} x_{6} x_{8} x_{9}+x_{3} x_{6} x_{7} x_{8} x_{9}\right) \text {, } \\
& \hat{X}[4]=\frac{\mathrm{A} 4 * \mathrm{~B} 4}{\mathrm{C} 4 * \mathrm{D} 4}
\end{aligned}
$$


$\mathrm{A} 4=\left(1+x_{5}+x_{4} x_{5}+x_{1} x_{4} x_{5}+x_{5} x_{6}+x_{4} x_{5} x_{6}+x_{1} x_{4} x_{5} x_{6}+x_{1} x_{2} x_{4} x_{5} x_{6}\right.$ $+x_{5} x_{6} x_{9}+x_{4} x_{5} x_{6} x_{9}+x_{1} x_{4} x_{5} x_{6} x_{9}+x_{1} x_{2} x_{4} x_{5} x_{6} x_{9}+x_{4} x_{5} x_{6} x_{8} x_{9}$ $\left.+x_{1} x_{4} x_{5} x_{6} x_{8} x_{9}+x_{1} x_{2} x_{4} x_{5} x_{6} x_{8} x_{9}+x_{1} x_{2} x_{4} x_{5}^{2} x_{6} x_{8} x_{9}\right)$, $\mathrm{B} 4=\left(1+x_{3}+x_{3} x_{6}+x_{3} x_{6} x_{9}+x_{3} x_{6} x_{8} x_{9}+x_{3} x_{6} x_{7} x_{8} x_{9}\right)$, $\mathrm{C} 4=x_{6}\left(1+x_{2}+x_{2} x_{3}+x_{2} x_{5}+x_{2} x_{3} x_{5}+x_{2} x_{4} x_{5}+x_{2} x_{3} x_{4} x_{5}+x_{2} x_{3} x_{5} x_{6}\right.$ $\left.+x_{2} x_{3} x_{4} x_{5} x_{6}+x_{2} x_{3} x_{5} x_{6} x_{9}+x_{2} x_{3} x_{4} x_{5} x_{6} x_{9}+x_{2} x_{3} x_{4} x_{5} x_{6} x_{8} x_{9}\right)$, $\mathrm{D} 4=\left(1+x_{9}+x_{8} x_{9}+x_{5} x_{8} x_{9}+x_{7} x_{8} x_{9}+x_{5} x_{7} x_{8} x_{9}+x_{4} x_{5} x_{7} x_{8} x_{9}\right.$ $\left.+x_{1} x_{4} x_{5} x_{7} x_{8} x_{9}\right)$,

$\hat{X}[5]=\frac{\mathrm{A} 5 * \mathrm{~B} 5}{\mathrm{C} 5 * \mathrm{D} 5}$

$\mathrm{A} 5=\left(1+x_{8}+x_{5} x_{8}+x_{5} x_{6} x_{8}+x_{7} x_{8}+x_{5} x_{7} x_{8}+x_{4} x_{5} x_{7} x_{8}+x_{1} x_{4} x_{5} x_{7} x_{8}\right.$ $\left.+x_{5} x_{6} x_{7} x_{8}+x_{4} x_{5} x_{6} x_{7} x_{8}+x_{1} x_{4} x_{5} x_{6} x_{7} x_{8}+x_{1} x_{2} x_{4} x_{5} x_{6} x_{7} x_{8}\right)$, $\mathrm{B} 5=\left(1+x_{2}+x_{2} x_{3}+x_{2} x_{5}+x_{2} x_{3} x_{5}+x_{2} x_{4} x_{5}+x_{2} x_{3} x_{4} x_{5}+x_{2} x_{3} x_{5} x_{6}\right.$ $\left.+x_{2} x_{3} x_{4} x_{5} x_{6}+x_{2} x_{3} x_{5} x_{6} x_{9}+x_{2} x_{3} x_{4} x_{5} x_{6} x_{9}+x_{2} x_{3} x_{4} x_{5} x_{6} x_{8} x_{9}\right)$, C5 $=x_{5}\left(1+x_{4}+x_{1} x_{4}+x_{1} x_{2} x_{4}+x_{1} x_{2} x_{3} x_{4}+x_{4} x_{8}+x_{1} x_{4} x_{8}+x_{1} x_{2} x_{4} x_{8}\right.$ $\left.+x_{1} x_{2} x_{3} x_{4} x_{8}+x_{1} x_{2} x_{4} x_{5} x_{8}+x_{1} x_{2} x_{3} x_{4} x_{5} x_{8}+x_{1} x_{2} x_{3} x_{4} x_{5} x_{6} x_{8}\right)$, $\mathrm{D} 5=\left(1+x_{6}+x_{2} x_{6}+x_{6} x_{9}+x_{2} x_{6} x_{9}+x_{6} x_{8} x_{9}+x_{2} x_{6} x_{8} x_{9}+x_{2} x_{5} x_{6} x_{8} x_{9}\right.$ $\left.+x_{6} x_{7} x_{8} x_{9}+x_{2} x_{6} x_{7} x_{8} x_{9}+x_{2} x_{5} x_{6} x_{7} x_{8} x_{9}+x_{2} x_{4} x_{5} x_{6} x_{7} x_{8} x_{9}\right)$,

$\hat{X}[6]=\frac{\mathrm{A} 6 * \mathrm{~B} 6}{\mathrm{C} 6 * \mathrm{D} 6}$

$\mathrm{A} 6=\left(1+x_{7}+x_{4} x_{7}+x_{1} x_{4} x_{7}+x_{1} x_{2} x_{4} x_{7}+x_{1} x_{2} x_{3} x_{4} x_{7}\right)$, $\mathrm{B} 6=\left(1+x_{5}+x_{4} x_{5}+x_{1} x_{4} x_{5}+x_{5} x_{6}+x_{4} x_{5} x_{6}+x_{1} x_{4} x_{5} x_{6}+x_{1} x_{2} x_{4} x_{5} x_{6}\right.$ $+x_{5} x_{6} x_{9}+x_{4} x_{5} x_{6} x_{9}+x_{1} x_{4} x_{5} x_{6} x_{9}+x_{1} x_{2} x_{4} x_{5} x_{6} x_{9}+x_{4} x_{5} x_{6} x_{8} x_{9}$ $\left.+x_{1} x_{4} x_{5} x_{6} x_{8} x_{9}+x_{1} x_{2} x_{4} x_{5} x_{6} x_{8} x_{9}+x_{1} x_{2} x_{4} x_{5}^{2} x_{6} x_{8} x_{9}\right)$, $\mathrm{C} 6=x_{4}\left(1+x_{8}+x_{5} x_{8}+x_{5} x_{6} x_{8}+x_{7} x_{8}+x_{5} x_{7} x_{8}+x_{4} x_{5} x_{7} x_{8}+x_{1} x_{4} x_{5} x_{7} x_{8}\right.$ $\left.+x_{5} x_{6} x_{7} x_{8}+x_{4} x_{5} x_{6} x_{7} x_{8}+x_{1} x_{4} x_{5} x_{6} x_{7} x_{8}+x_{1} x_{2} x_{4} x_{5} x_{6} x_{7} x_{8}\right)$, $\mathrm{D} 6=1+x_{1}+x_{1} x_{2}+x_{1} x_{2} x_{3}+x_{1} x_{2} x_{5}+x_{1} x_{2} x_{3} x_{5}+x_{1} x_{2} x_{3} x_{5} x_{6}$ $\left.+x_{1} x_{2} x_{3} x_{5} x_{6} x_{9}\right)$,

$\hat{X}[8]=\frac{\mathrm{A} 8 * \mathrm{~B} 8}{\mathrm{C} 8 * \mathrm{D} 8}$

$\mathrm{A} 8=\left(1+x_{4}+x_{1} x_{4}+x_{1} x_{2} x_{4}+x_{1} x_{2} x_{3} x_{4}+x_{4} x_{8}+x_{1} x_{4} x_{8}+x_{1} x_{2} x_{4} x_{8}\right.$ $\left.+x_{1} x_{2} x_{3} x_{4} x_{8}+x_{1} x_{2} x_{4} x_{5} x_{8}+x_{1} x_{2} x_{3} x_{4} x_{5} x_{8}+x_{1} x_{2} x_{3} x_{4} x_{5} x_{6} x_{8}\right)$, $\mathrm{B} 8=\left(1+x_{9}+x_{8} x_{9}+x_{5} x_{8} x_{9}+x_{7} x_{8} x_{9}+x_{5} x_{7} x_{8} x_{9}+x_{4} x_{5} x_{7} x_{8} x_{9}\right.$ $\left.+x_{1} x_{4} x_{5} x_{7} x_{8} x_{9}\right)$, $\mathrm{C} 8=\left(1+x_{7}+x_{4} x_{7}+x_{1} x_{4} x_{7}+x_{1} x_{2} x_{4} x_{7}+x_{1} x_{2} x_{3} x_{4} x_{7}\right) x_{8}$, $\mathrm{D} 8=\left(1+x_{5}+x_{4} x_{5}+x_{1} x_{4} x_{5}+x_{5} x_{6}+x_{4} x_{5} x_{6}+x_{1} x_{4} x_{5} x_{6}+x_{1} x_{2} x_{4} x_{5} x_{6}\right.$ $+x_{5} x_{6} x_{9}+x_{4} x_{5} x_{6} x_{9}+x_{1} x_{4} x_{5} x_{6} x_{9}+x_{1} x_{2} x_{4} x_{5} x_{6} x_{9}+x_{4} x_{5} x_{6} x_{8} x_{9}$ $\left.+x_{1} x_{4} x_{5} x_{6} x_{8} x_{9}+x_{1} x_{2} x_{4} x_{5} x_{6} x_{8} x_{9}+x_{1} x_{2} x_{4} x_{5}^{2} x_{6} x_{8} x_{9}\right)$, 


\section{A.4. $A_{2}$ pentagon}

The quiver is shown in Figure A4, and the maximal mutation sequence is

$$
\left(\mu_{2}, \mu_{4}, \mu_{1}, \mu_{5}\right), \quad\left(\mu_{3}, \mu_{6}, \mu_{2}, \mu_{7}\right),\left(\mu_{3}, \mu_{4}, \mu_{6}\right)
$$

So the final cluster coordinates are

$$
\begin{aligned}
& \hat{x}[1]=\frac{1+x_{5}+x_{3} x_{5}+x_{4} x_{5}+x_{1} x_{4} x_{5}+x_{3} x_{4} x_{5}+x_{1} x_{3} x_{4} x_{5}+x_{1} x_{2} x_{3} x_{4} x_{5}+x_{3} x_{5} x_{7}+x_{3} x_{4} x_{5} x_{7}+x_{1} x_{3} x_{4} x_{5} x_{7}+x_{1} x_{2} x_{3} x_{4} x_{5} x_{7}}{x_{4}\left(1+x_{1}+x_{1} x_{2}+x_{1} x_{2} x_{5}\right)}, \\
& \hat{x}[2]=\frac{1+x_{7}+x_{6} x_{7}+x_{5} x_{6} x_{7}+x_{4} x_{5} x_{6} x_{7}+x_{1} x_{4} x_{5} x_{6} x_{7}}{x_{6}\left(1+x_{5}+x_{3} x_{5}+x_{4} x_{5}+x_{1} x_{4} x_{5}+x_{3} x_{4} x_{5}+x_{1} x_{9} x_{4} x_{5}+x_{1} x_{2} x_{9} x_{4} x_{5}+x_{3} x_{5} x_{7}+x_{3} x_{6} x_{7}\right.}
\end{aligned}
$$

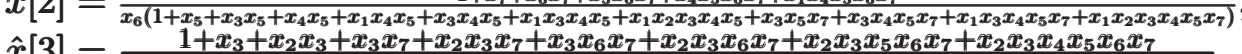

$$
\begin{aligned}
& \hat{x}[3]=\frac{1+x_{3}+x_{2} x_{3}+x_{3} x_{7}+x_{2} x_{3} x_{7}+x_{3} x_{6} x_{7}+x_{2} x_{3} x_{6} x_{7}+x_{2} x_{3} x_{5} x_{6} x_{7}+x_{2} x_{3} x_{4} x_{5} x_{6} x_{7}}{\left(1+x_{6}+x_{5} x_{6}+x_{3} x_{5} x_{6}+x_{4} x_{5} x_{6}+x_{1} x_{4} x_{5} x_{6}+x_{3} x_{4} x_{5} x_{6}+x_{1} x_{3} x_{4} x_{5} x_{6}+x_{1} x_{2} x_{3} x_{4} x_{5} x_{6}\right) x_{7}}, \\
& \hat{x}[4]=\frac{1+x_{4}+x_{1} x_{4}+x_{1} x_{2} x_{4}}{x_{1}\left(1+x_{2}+x_{2} x_{5}+x_{2} x_{4} x_{5}\right)} \text {, } \\
& \hat{x}[5]=\frac{\left(1+x_{1}+x_{1} x_{2}+x_{1} x_{2} x_{5}\right)\left(1+x_{3}+x_{2} x_{3}+x_{3} x_{7}+x_{2} x_{3} x_{7}+x_{3} x_{6} x_{7}+x_{2} x_{3} x_{6} x_{7}+x_{2} x_{3} x_{5} x_{6} x_{7}+x_{2} x_{3} x_{4} x_{5} x_{6} x_{7}\right)}{x_{2}\left(1+x_{5}+x_{3} x_{5}+x_{4} x_{5}+x_{1} x_{4} x_{5}+x_{3} x_{4} x_{5}+x_{1} x_{3} x_{4} x_{5}+x_{1} x_{2} x_{3} x_{4} x_{5}+x_{3} x_{5} x_{7}+x_{3} x_{4} x_{5} x_{7}+x_{1} x_{3} x_{4} x_{5} x_{7}+x_{1} x_{2} x_{3} x_{4} x_{5} x_{7}\right)}, \\
& \hat{x}[6]=\frac{\left(1+x_{2}+x_{2} x_{5}+x_{2} x_{5} x_{5}\right)\left(1+x_{6}+x_{5} x_{6}+x_{3} x_{5} x_{6}+x_{4} x_{5} x_{6}+x_{1} x_{4} x_{5} x_{6}+x_{3} x_{4} x_{5} x_{6}+x_{1} x_{3} x_{4} x_{5} x_{6}+x_{1} x_{2} x_{3} x_{4} x_{5} x_{6}\right)}{\left(1+x_{4}+x_{1} x_{4}+x_{1} x_{2} x_{4}\right) x_{5}\left(1+x_{3}+x_{2} x_{3}+x_{3} x_{7}+x_{2} x_{3} x_{7}+x_{3} x_{6} x_{7}+x_{2} x_{3} x_{6} x_{7}+x_{2} x_{3} x_{5} x_{6} x_{7}+x_{2} x_{3} x_{4} x_{5} x_{6} x_{7}\right)}, \\
& \hat{x}[7]=\frac{1+x_{5}+x_{3} x_{5}+x_{4} x_{5}+x_{1} x_{4} x_{5}+x_{3} x_{4} x_{5}+x_{1} x_{3} x_{4} x_{5}+x_{1} x_{2} x_{3} x_{4} x_{5}+x_{3} x_{5} x_{7}+x_{3} x_{4} x_{5} x_{7}+x_{1} x_{3} x_{4} x_{5} x_{7}+x_{1} x_{2} x_{3} x_{4} x_{5} x_{7}}{x_{3}\left(1+x_{2}+x_{2} x_{5}+x_{2} x_{4} x_{5}\right)\left(1+x_{7}+x_{6} x_{7}+x_{5} x_{6} x_{7}+x_{4} x_{5} x_{6} x_{7}+x_{1} x_{4} x_{5} x_{6} x_{7}\right)} .
\end{aligned}
$$

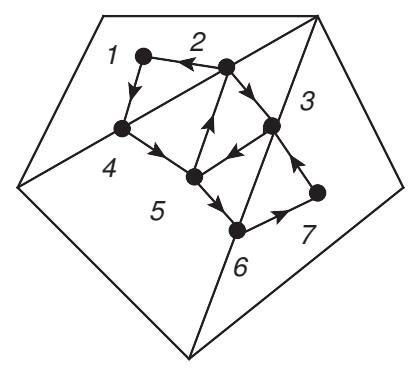

Figure A4: The quiver for $A_{2}$ pentagon with all punctures full.

\section{A.5. $S U(2)$ with four flavors}

The quiver is shown in Figure A5. The maximal green mutation sequences are

$$
\mu_{1}, \mu_{2}, \mu_{3}, \mu_{4}, \mu_{5}, \mu_{6}, \mu_{1}, \mu_{2}, \mu_{3}, \mu_{4}, \mu_{5}, \mu_{6}
$$

So the spectral generator is

$\hat{x}[1]=\frac{\mathrm{A} 1}{\mathrm{~B} 1}$

$\mathrm{A} 1=\left(1+x_{6}+x_{1} x_{6}+x_{4} x_{6}+x_{1} x_{4} x_{6}+x_{1} x_{5} x_{6}+x_{4} x_{5} x_{6}+2 x_{1} x_{4} x_{5} x_{6}\right.$

$+x_{1} x_{2} x_{4} x_{5} x_{6}+x_{1} x_{3} x_{4} x_{5} x_{6}+x_{1} x_{4} x_{5}^{2} x_{6}+x_{1} x_{2} x_{4} x_{5}^{2} x_{6}$

$\left.+x_{1} x_{3} x_{4} x_{5}^{2} x_{6}+x_{1} x_{2} x_{3} x_{4} x_{5}^{2} x_{6}+x_{1} x_{2} x_{3} x_{4} x_{5}^{2} x_{6}^{2}\right)$, 


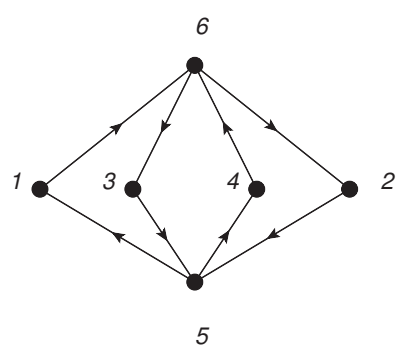

Figure A5: The quiver for $S U(2)$ with four flavors.

$$
\begin{aligned}
& \mathrm{B} 1=x_{4}\left(1+x_{5}+x_{2} x_{5}+x_{3} x_{5}+x_{2} x_{3} x_{5}+x_{2} x_{5} x_{6}+x_{3} x_{5} x_{6}+2 x_{2} x_{3} x_{5} x_{6}\right. \\
& +x_{1} x_{2} x_{3} x_{5} x_{6}+x_{2} x_{3} x_{4} x_{5} x_{6}+x_{2} x_{3} x_{5} x_{6}^{2}+x_{1} x_{2} x_{3} x_{5} x_{6}^{2}+x_{2} x_{3} x_{4} x_{5} x_{6}^{2} \\
& \left.+x_{1} x_{2} x_{3} x_{4} x_{5} x_{6}^{2}+x_{1} x_{2} x_{3} x_{4} x_{5}^{2} x_{6}^{2}\right) \text {, } \\
& \hat{x}[2]=\frac{\mathrm{A} 2}{\mathrm{~B} 2} \\
& \mathrm{~A} 2=\left(1+x_{5}+x_{2} x_{5}+x_{3} x_{5}+x_{2} x_{3} x_{5}+x_{2} x_{5} x_{6}+x_{3} x_{5} x_{6}+2 x_{2} x_{3} x_{5} x_{6}\right. \\
& +x_{1} x_{2} x_{3} x_{5} x_{6}+x_{2} x_{3} x_{4} x_{5} x_{6}+x_{2} x_{3} x_{5} x_{6}^{2}+x_{1} x_{2} x_{3} x_{5} x_{6}^{2}+x_{2} x_{3} x_{4} x_{5} x_{6}^{2} \\
& \left.+x_{1} x_{2} x_{3} x_{4} x_{5} x_{6}^{2}+x_{1} x_{2} x_{3} x_{4} x_{5}^{2} x_{6}^{2}\right) \text {, } \\
& \mathrm{B} 2=x_{3}\left(1+x_{6}+x_{1} x_{6}+x_{4} x_{6}+x_{1} x_{4} x_{6}+x_{1} x_{5} x_{6}+x_{4} x_{5} x_{6}+2 x_{1} x_{4} x_{5} x_{6}\right. \\
& +x_{1} x_{2} x_{4} x_{5} x_{6}+x_{1} x_{3} x_{4} x_{5} x_{6}+x_{1} x_{4} x_{5}^{2} x_{6}+x_{1} x_{2} x_{4} x_{5}^{2} x_{6}+x_{1} x_{3} x_{4} x_{5}^{2} x_{6} \\
& \left.+x_{1} x_{2} x_{3} x_{4} x_{5}^{2} x_{6}+x_{1} x_{2} x_{3} x_{4} x_{5}^{2} x_{6}^{2}\right) \text {, } \\
& \hat{x}[3]=\frac{\mathrm{A} 3}{\mathrm{~B} 3} \\
& \mathrm{~A} 3=\left(1+x_{5}+x_{2} x_{5}+x_{3} x_{5}+x_{2} x_{3} x_{5}+x_{2} x_{5} x_{6}+x_{3} x_{5} x_{6}+2 x_{2} x_{3} x_{5} x_{6}\right. \\
& +x_{1} x_{2} x_{3} x_{5} x_{6}+x_{2} x_{3} x_{4} x_{5} x_{6}+x_{2} x_{3} x_{5} x_{6}^{2}+x_{1} x_{2} x_{3} x_{5} x_{6}^{2}+x_{2} x_{3} x_{4} x_{5} x_{6}^{2} \\
& \left.+x_{1} x_{2} x_{3} x_{4} x_{5} x_{6}^{2}+x_{1} x_{2} x_{3} x_{4} x_{5}^{2} x_{6}^{2}\right) \text {, } \\
& \mathrm{B} 3=x_{2}\left(1+x_{6}+x_{1} x_{6}+x_{4} x_{6}+x_{1} x_{4} x_{6}+x_{1} x_{5} x_{6}+x_{4} x_{5} x_{6}+2 x_{1} x_{4} x_{5} x_{6}\right. \\
& +x_{1} x_{2} x_{4} x_{5} x_{6}+x_{1} x_{3} x_{4} x_{5} x_{6}+x_{1} x_{4} x_{5}^{2} x_{6}+x_{1} x_{2} x_{4} x_{5}^{2} x_{6}+x_{1} x_{3} x_{4} x_{5}^{2} x_{6} \\
& \left.+x_{1} x_{2} x_{3} x_{4} x_{5}^{2} x_{6}+x_{1} x_{2} x_{3} x_{4} x_{5}^{2} x_{6}^{2}\right) \text {, } \\
& \hat{x}[4]=\frac{\mathrm{A} 4}{\mathrm{~B} 4} \\
& \mathrm{~A} 4=\left(1+x_{6}+x_{1} x_{6}+x_{4} x_{6}+x_{1} x_{4} x_{6}+x_{1} x_{5} x_{6}+x_{4} x_{5} x_{6}+2 x_{1} x_{4} x_{5} x_{6}\right. \\
& +x_{1} x_{2} x_{4} x_{5} x_{6}+x_{1} x_{3} x_{4} x_{5} x_{6}+x_{1} x_{4} x_{5}^{2} x_{6}+x_{1} x_{2} x_{4} x_{5}^{2} x_{6}+x_{1} x_{3} x_{4} x_{5}^{2} x_{6} \\
& \left.+x_{1} x_{2} x_{3} x_{4} x_{5}^{2} x_{6}+x_{1} x_{2} x_{3} x_{4} x_{5}^{2} x_{6}^{2}\right) \text {, } \\
& \mathrm{B} 4=x_{1}\left(1+x_{5}+x_{2} x_{5}+x_{3} x_{5}+x_{2} x_{3} x_{5}+x_{2} x_{5} x_{6}+x_{3} x_{5} x_{6}+2 x_{2} x_{3} x_{5} x_{6}\right. \\
& +x_{1} x_{2} x_{3} x_{5} x_{6}+x_{2} x_{3} x_{4} x_{5} x_{6}+x_{2} x_{3} x_{5} x_{6}^{2}+x_{1} x_{2} x_{3} x_{5} x_{6}^{2}+x_{2} x_{3} x_{4} x_{5} x_{6}^{2} \\
& \left.+x_{1} x_{2} x_{3} x_{4} x_{5} x_{6}^{2}+x_{1} x_{2} x_{3} x_{4} x_{5}^{2} x_{6}^{2}\right) \text {, } \\
& \hat{x}[5]=\frac{\mathrm{A} 5 * \mathrm{C} 5}{\mathrm{~B} 5 * \mathrm{D} 5}
\end{aligned}
$$




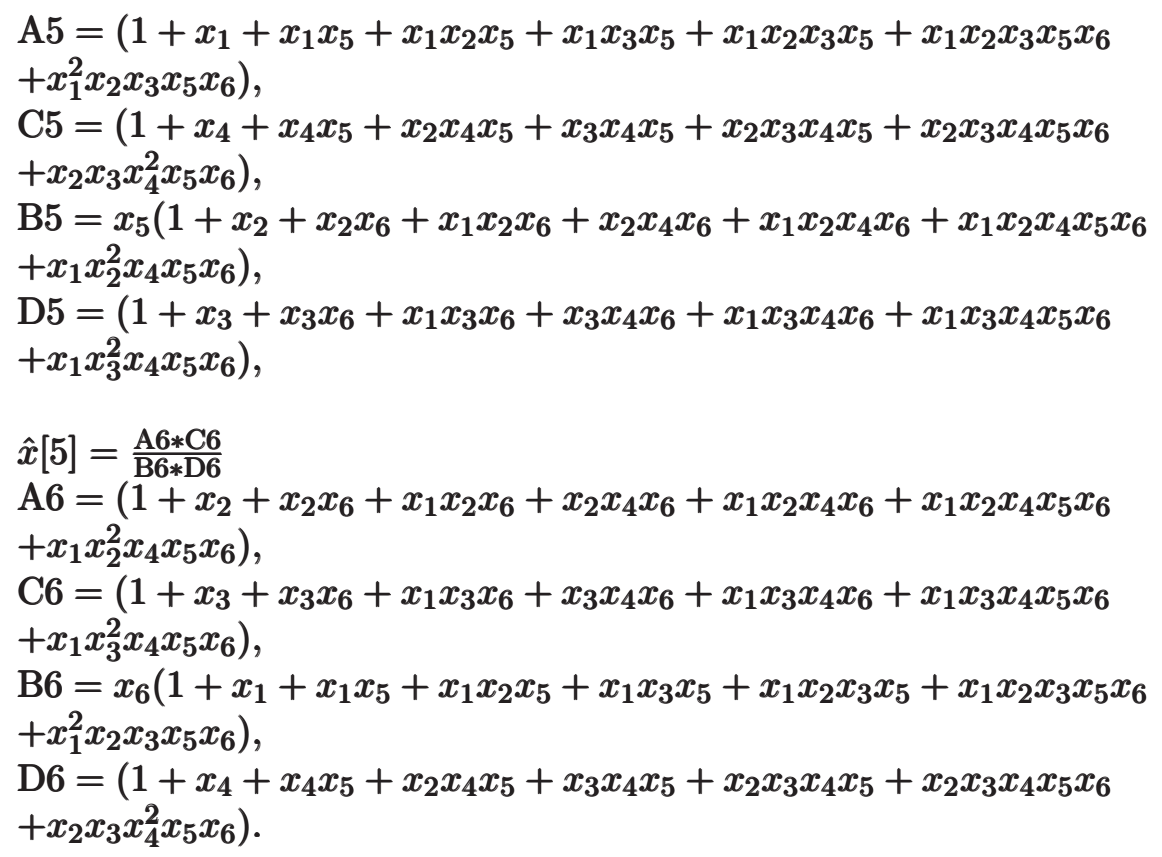

\section{Appendix B. Refined spectral generator}

Let's describe a simple way of finding the refined spectral generator from the classical spectral generator. The $q$ deformation of the cluster algebra is

$$
X_{i} X_{j}=q^{\epsilon_{i j}} X_{i} X_{j}
$$

where $\epsilon_{i j}$ is the antisymmetric tensor read from the quiver, and the cluster transformation on quantum cluster algebra is

$$
\begin{aligned}
& X_{k}^{\prime}=X_{k}^{-1} \\
& X_{i}^{\prime}=X_{i}\left(\prod_{a=1}^{\left|\epsilon_{i k}\right|}\left(1+q^{a-1 / 2} X_{k}^{-\operatorname{sgn}\left(\epsilon_{i k}\right)}\right)\right)^{-\operatorname{sgn}\left(\epsilon_{i k}\right)} .
\end{aligned}
$$

Given the mutation sequences, one could find the final quantum cluster coordinates.

In practice, there is a way of reading the quantum cluster algebra from the classical one using the $*$ invariance. One could define a $*$ action on the quantum cluster algebra

$$
*(q)=q^{-1}, \quad *\left(X_{i}\right)=X_{i}, \quad *\left(X_{i} X_{j}\right)=*\left(X_{j}\right) *\left(X_{i}\right)=X_{j} X_{i},
$$


and any monomial appearing in the quantum cluster algebra should be invariant under this $*$ action, this would uniquely fix the $q$ factor before each monomial. So we will first find the classical spectral generator and then find the quantum version by adding the $q$ factor before each monomial to make it $*$ invariant.
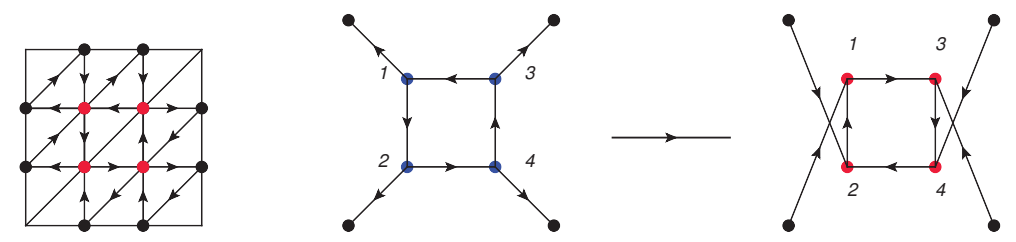

Figure B1: The quiver and maximal green mutation for a quiver from a disc with four $A_{2}$ full punctures.

Let's look at an example which is represented by a $A_{2}$ theory on a disc with four full punctures. The quiver and the initial and final configuration of the maximal green mutation sequences is shown in Figure B1. The maximal green mutation sequences are

$$
\mu_{2}, \mu_{3}, \mu_{1}, \mu_{4}, \mu_{2}, \mu_{3}
$$

and the final cluster coordinates are

$$
\begin{aligned}
& \hat{X}[1]=\frac{1+x_{4}+x_{2} x_{4}+x_{1} x_{2} x_{4}}{x_{2}\left(1+x_{1}+x_{1} x_{3}+x_{1} x_{3} x_{4}\right)}, \\
& \hat{X}[2]=\frac{1+x_{2}+x_{1} x_{2}+x_{1} x_{2} x_{3}}{x_{1}\left(1+x_{3}+x_{3} x_{4}+x_{2} x_{3} x_{4}\right)}, \\
& \hat{X}[3]=\frac{1+x_{3}+x_{3} x_{4}+x_{2} x_{3} x_{4}}{\left(1+x_{2}+x_{1} x_{2}+x_{1} x_{2} x_{3} x_{4}\right.}, \\
& \hat{X}[4]=\frac{1+x_{1}+x_{1} x_{3}+x_{1} x_{3} x_{4}}{x_{3}\left(1+x_{4}+x_{2} x_{4}+x_{1} x_{2} x_{4}\right)} .
\end{aligned}
$$

Using the $*$ invariance, we can easily find the refined spectral generator:

$$
\begin{aligned}
& \hat{X}[1]=\frac{1}{x_{2}+q^{-1 / 2} x_{1} x_{2}+x_{1} x_{2} x_{3}+x_{1} x_{2} x_{3} x_{4}}+\frac{1}{q^{1 / 2} x_{2} x_{4}{ }^{1}+x_{1} x_{2} x_{4}=1+x_{1} x_{2} x_{3} x_{4}{ }^{-1}+x_{1} x_{2} x_{3}} \\
& +\frac{1}{x_{4}^{-1}+x_{1} x_{4}^{-1}+x_{1} x_{3} x_{4}^{-1}+q^{1 / 2} x_{1} x_{3}}+\frac{1}{x_{1}^{-1} x_{4}^{-1}+x_{4}^{-1}+q^{-1 / 2} x_{3} x_{4}^{-1}+x_{3}} \text {, } \\
& \hat{X}[2]=\frac{1}{x_{1}+q^{1 / 2} x_{1} x_{3}+q x_{1} x_{3} x_{4}+x_{1} x_{2} x_{3} x_{4}}+\frac{1}{q^{1 / 2} x_{1} x_{2}^{-1}+q x_{1} x_{2}^{-1} x_{3}+q^{2} x_{1} x_{2}^{-1} x_{3} x_{4}+q x_{1} x_{3} x_{4}} \\
& +\frac{1}{x_{2}^{-1}+x_{2}^{-1} x_{3}+q x_{2}^{-1} x_{3} x_{4}+q^{1 / 2} x_{3} x_{4}}+\frac{1}{x_{2}^{-1} x_{3}^{-1}+x_{2}^{-1}+q^{1 / 2} x_{2} x_{4}^{-1}+x_{4}} \text {, } \\
& \hat{X}[3]=\frac{1}{x_{4}+q^{-1 / 2} x_{2} x_{4}+q^{-1} x_{1} x_{2} x_{4}+x_{1} x_{2} x_{3} x_{4}} \\
& +\frac{1}{q^{-1 / 2} x_{3}^{-1} x_{4}+q^{-1} x_{2} x_{3}^{-1} x_{4}+q^{-2} x_{1} x_{2} x_{3}^{-1} x_{4}+q^{-1} x_{1} x_{2} x_{4}} \\
& +\frac{1}{x_{3}^{-1}+x_{2} x_{3}^{-1}+q^{-1} x_{1} x_{2} x_{3}^{-1}+x_{1} x_{2}}+\frac{1}{x_{2}^{-1} x_{3}^{-1}+x_{3}^{-1}+q^{-1 / 2} x_{1} x_{3}^{-1}+x_{1}}, \\
& \hat{X}[4]=\frac{1}{x_{3}+q^{1 / 2} x_{3} x_{4}+x_{2} x_{3} x_{4}+x_{1} x_{2} x_{3} x_{4}}+\frac{1}{q^{-1 / 2} x_{1}^{-1} x_{3}+x_{1}{ }^{-1} x_{3} x_{4}+x_{1}{ }^{-1} x_{2} x_{3} x_{4}+x_{2} x_{3} x_{4}} \\
& +\frac{1}{x_{1}^{-1}+x_{1}^{-1} x_{4}+x_{1}^{-1} x_{2} x_{4}+q^{-1 / 2} x_{2} x_{4}}+\frac{q}{x_{1}^{-1} x_{4}^{-1}+x_{1}^{-1}+q^{1 / 2} x_{1}^{-1} x_{2}+x_{2}} \text {. }
\end{aligned}
$$




\section{References}

[1] C. Montonen and D. I. Olive, Magnetic monopoles as gauge particles? Phys. Lett. B72 (1977), 117.

[2] N. Seiberg and E. Witten, Monopole condensation, and confinement in $N=2$ supersymmetric Yang-Mills theory, Nucl. Phys. B426 (1994), 19-52, arXiv:hep-th/9407087.

[3] N. Seiberg and E. Witten, Monopoles, duality and chiral symmetry breaking in $N=2$ supersymmetric QCD, Nucl. Phys. B431 (1994), 484550, arXiv:hep-th/9408099 [hep-th].

[4] F. Ferrari and A. Bilal, The Strong coupling spectrum of the SeibergWitten theory, Nucl. Phys. B469 (1996), 387-402, arXiv:hep-th/ 9602082 [hep-th].

[5] A. Bilal and F. Ferrari, Curves of marginal stability, and weak and strong coupling BPS spectra in $N=2$ supersymmetric $Q C D$, Nucl. Phys. B480 (1996), 589-622, arXiv:hep-th/9605101 [hep-th].

[6] A. Bilal and F. Ferrari, The BPS spectra and superconformal points in massive $N=2$ supersymmetric QCD, Nucl. Phys. B516 (1998), 175228, arXiv:hep-th/9706145 [hep-th].

[7] F. Ferrari, The Dyon spectra of finite gauge theories, Nucl. Phys. B501 (1997), 53-96, arXiv:hep-th/9702166 [hep-th].

[8] M. Kontsevich and Y. Soibelman, Stability structures, motivic Donaldson-Thomas invariants and cluster transformations, arXiv: 0811.2435 [math.AG].

[9] D. Joyce and Y. Song, A theory of generalized Donaldson-Thomas invariants, arXiv:0810.5645.

[10] M. Kontsevich and Y. Soibelman, Cohomological Hall algebra, exponential Hodge structures and motivic Donaldson-Thomas invariants, Commun. Num. Theor. Phys. 5 (2011), 231-352, arXiv:1006.2706 [math.AG].

[11] D. Gaiotto, G. W. Moore, and A. Neitzke, Four-dimensional wallcrossing via three-dimensional field theory, Commun. Math. Phys. 299 (2010), 163-224, arXiv:0807.4723 [hep-th].

[12] F. Denef and G. W. Moore, Split states, entropy enigmas, holes and halos, JHEP 1111 (2011) 129, arXiv:hep-th/0702146 [hep-th]. 
[13] D. Gaiotto, G. W. Moore, and A. Neitzke, Wall-crossing, Hitchin systems, and the WKB approximation, arXiv:0907.3987 [hep-th].

[14] S. Cecotti and C. Vafa, BPS wall crossing and topological strings, arXiv:0910.2615 [hep-th].

[15] T. Dimofte and S. Gukov, Refined, motivic, and quantum, Lett. Math. Phys. 91 (2010) 1, arXiv:0904.1420 [hep-th].

[16] S. Cecotti, A. Neitzke, and C. Vafa, R-twisting and 4d/2d correspondences, arXiv:1006.3435 [hep-th].

[17] W.-y. Chuang, D.-E. Diaconescu, and G. Pan, Wallcrossing and cohomology of the moduli space of Hitchin pairs, Commun. Num. Theor. Phys. 5 (2011), 1-56, arXiv:1004.4195 [math.AG].

[18] D. Gaiotto, G. W. Moore, and A. Neitzke, Framed BPS states, arXiv: 1006.0146 [hep-th].

[19] D. Gaiotto, G. W. Moore, and A. Neitzke, Wall-crossing in coupled 2d-4d systems, arXiv:1103.2598 [hep-th].

[20] M. Alim, S. Cecotti, C. Cordova, S. Espahbodi, A. Rastogi, and C. Vafa, $B P S$ quivers and spectra of complete $N=2$ quantum field theories, arXiv:1109.4941 [hep-th].

[21] M. Alim, S. Cecotti, C. Cordova, S. Espahbodi, A. Rastogi, and C. Vafa, $N=2$ quantum field theories and their BPS quivers, arXiv:1112.3984 [hep-th].

[22] D. Gaiotto, G. W. Moore, and A. Neitzke, Spectral networks, arXiv: 1204.4824 [hep-th].

[23] D. Gaiotto, G. W. Moore, and A. Neitzke, Spectral networks and snakes, arXiv:1209.0866 [hep-th].

[24] D. Gaiotto, $\mathcal{N}=2$ dualities, arXiv:0904.2715 [hep-th].

[25] D. Xie, General Argyres-Douglas theory, arXiv:1204.2270 [hep-th].

[26] D. Nanopoulos and D. Xie, $N=2$ generalized superconformal quiver gauge theory, JHEP 1209 (2012) 127, arXiv:1006.3486 [hep-th].

[27] P. C. Argyres and M. R. Douglas, New phenomena in SU(3) supersymmetric gauge theory, Nucl.Phys. B448 (1995), 93-126, arXiv: hep-th/9505062 [hep-th]. 
[28] P. C. Argyres, M. Ronen Plesser, N. Seiberg, and E. Witten, New $N=$ 2 superconformal field theories in four-dimensions, Nucl. Phys. B461 (1996), 71-84, arXiv:hep-th/9511154 [hep-th].

[29] A. Gadde, L. Rastelli, S. S. Razamat, and W. Yan, Gauge theories and Macdonald polynomials, arXiv:1110.3740 [hep-th].

[30] F. Benini, Y. Tachikawa, and D. Xie, Mirrors of 3d Sicilian theories, JHEP 1009 (2010) 063, arXiv:1007.0992 [hep-th].

[31] S. Cecotti and C. Vafa, Classification of complete $N=2$ supersymmetric theories in 4 dimensions, arXiv:1103.5832 [hep-th].

[32] H.-Y. Chen, N. Dorey, and K. Petunin, Moduli Space and Wall-Crossing Formulae in Higher-Rank Gauge Theories, JHEP 1111 (2011) 020, arXiv:1105.4584 [hep-th].

[33] V. V. Fock and A. B. Goncharov, Moduli spaces of local systems and higher teichmuller theory, arXiv:math/0311149.

[34] D. Xie, Network, Cluster coordinates and $N=2$ theory I, arXiv: 1203.4573 [hep-th].

[35] D. Xie, Network, cluster coordinates and $N=2$ theory II: Irregular singularity, arXiv:1207.6112 [hep-th].

[36] M. Reineke, Poisson automorphisms and quiver moduli, J. Inst. Math. Jussieu 9 (2010), no. 3, 653-667.

[37] B. Keller, On cluster theory and quantum dilogarithm identities, arXiv: 1102.4148.

[38] B. Keller, Quiver mutation in Java,. www.math.jussied.fr/ keller/ quivermutation.

[39] L. Faddeev and R. Kashaev, Quantum dilogarithm, Mod. Phys. Lett. A9 (1994), 427-434, arXiv:hep-th/9310070 [hep-th].

[40] E. Kiritsis, Introduction to nonperturbative string theory, arXiv:hepth/9708130 [hep-th].

[41] M. R. Douglas, B. Fiol, and C. Romelsberger, Stability and BPS branes, JHEP 0509 (2005) 006, arXiv:hep-th/0002037 [hep-th].

[42] F. Denef, Quantum quivers and Hall / hole halos, JHEP 0210 (2002) 023, arXiv:hep-th/0206072 [hep-th]. 
[43] B. Fiol, The BPS spectrum of $N=2 S U(N) S Y M$, JHEP 0602 (2006) 065.

[44] S. Fomin and A. Zelevinsky, Cluster algebras I: Foundations, J. Amer. Math. Soc. 15 (2002), 497-529, arXiv:math/0104151.

[45] N. Seiberg, Electric-magnetic duality in supersymmetric nonAbelian gauge theories, Nucl. Phys. B435 (1995) 129-146, arXiv:hep-th/ 9411149 [hep-th].

[46] H. Derksen, J. Weyman, and A. Zelevinsky, Quivers with potentials and their representations $i$ : Mutations, arXiv:0704.0649.

[47] M. Reineke, Moduli of representations of quivers, arXiv:0802.2147.

[48] K. A, Moduli of representations of finite dimensional algebras, The quarterly journal of mathematics 45 (4) (1994), 515-530.

[49] T. Dimofte, S. Gukov, and Y. Soibelman, Quantum wall crossing in $N=2$ gauge theories, Lett. Math. Phys. 95 (2011), 1-25, arXiv: 0912.1346 [hep-th].

[50] H. Kim, J. Park, Z. Wang, and P. Yi, Ab initio wall-crossing, JHEP 1109 (2011) 079, arXiv:1107.0723 [hep-th].

[51] S. Alexandrov, D. Persson, and B. Pioline, Wall-crossing, Rogers dilogarithm, and the $Q K / H K$ correspondence, JHEP 1112 (2011) 027, arXiv:1110.0466 [hep-th].

[52] S.-J. Lee, Z.-L. Wang, and P. Yi, Quiver invariants from intrinsic Higgs states, JHEP 1207 (2012) 169, arXiv:1205.6511 [hep-th].

[53] S.-J. Lee, Z.-L. Wang, and P. Yi, BPS states, refined indices, and quiver invariants, JHEP 1210 (2012) 094, arXiv:1207.0821 [hep-th].

[54] K. Nagao, Wall-crossing of the motivic Donaldson-Thomas invariants, arXiv:1103.2922.

[55] S. Mozgovoy, On the motivic Donaldson-Thomas invariants of quivers with potentials, arXiv:1103.2902.

[56] S. Fomin and A. Zelevinsky, Cluster algebras iv: Coefficients, arXiv: math/0602259.

[57] V. V. Fock and A. B. Goncharov, Cluster ensembles, quantization and the dilogarithm, arXiv:math/0311245. 
[58] V. V. Fock and A. B. Goncharov, The quantum dilogarithm and representations quantum cluster varieties, arXiv:math/0702397.

[59] R. M. Kashaev and T. Nakanishi, Classical and quantum dilogarithm identities, arXiv:1104.4630.

[60] E. H. Saidi, Mutation symmetries in BPS quiver theories: building the BPS spectra, JHEP 1208 (2012) 018, arXiv:1204.0395 [hep-th].

[61] S. Fomin, M. Shapiro, and D. Thurston, Cluster algebras and triangulated surfaces. part $i$ : Cluster complexes, Acta Mathematica 201 (2008) 83, arXiv:math/0608367.

[62] D. Labardini-Fragoso, Quivers with potentials associated to triangulated surfaces, arXiv:0803.1328.

[63] I. Assem, T. Brüstle, G. Charbonneau-Jodoin, and P.-G. Plamondon, Gentle algebras arising from surface triangulations, arXiv:0903.3347.

[64] T. Brüstle, G. Dupont, and M. Pérotin, On maximal green sequences, arXiv:1205.2050.

[65] D. Nanopoulos and D. Xie, Hitchin equation, singularity, and $\mathcal{N}=2$ superconformal field theories, JHEP 03 (2010) 043, arXiv:0911.1990 [hep-th].

[66] P. C. Argyres and N. Seiberg, S-Duality in $\mathcal{N}=2$ supersymmetric gauge theories, JHEP 12 (2007) 088, arXiv:0711.0054 [hep-th].

[67] F. Benini, S. Benvenuti, and Y. Tachikawa, Webs of five-Branes and $\mathcal{N}=2$ superconformal field theories, JHEP 09 (2009) 052, arXiv: 0906.0359 [hep-th].

[68] W. Lerche, On a boundary CFT description of nonperturbative $N=2$ Yang-Mills theory, arXiv:hep-th/0006100 [hep-th].

[69] A. Klemm, W. Lerche, P. Mayr, C. Vafa, and N. P. Warner, Selfdual strings and $N=2$ supersymmetric field theory, Nucl. Phys. B477 (1996), 746-766, arXiv:hep-th/9604034 [hep-th].

[70] A. D. Shapere and C. Vafa, BPS structure of Argyres-Douglas superconformal theories, arXiv:hep-th/9910182.

[71] J. J. Heckman, C. Vafa, D. Xie, and M. Yamazaki, String theory origin of bipartite SCFTs, arXiv:1211.4587 [hep-th]. 
[72] W.-y. Chuang and D. L. Jafferis, Wall crossing of BPS states on the conifold from seiberg duality and pyramid partitions, Commun. Math. Phys. 292 (2009), 285-301, arXiv:0810.5072 [hep-th].

[73] D. L. Jafferis and G. W. Moore, Wall crossing in local Calabi Yau manifolds, arXiv:0810.4909 [hep-th].

[74] M. Aganagic and M. Yamazaki, Open BPS wall crossing and M-theory, Nucl. Phys. B834 (2010), 258-272, arXiv:0911.5342 [hep-th].

[75] M. Aganagic and K. Schaeffer, Wall crossing, quivers and crystals, JHEP 1210 (2012) 153, arXiv:1006.2113 [hep-th].

[76] M. Yamazaki, Crystal melting and wall crossing phenomena, Int. J. Mod. Phys. A26 (2011), 1097-1228, arXiv:1002.1709 [hep-th].

[77] E. Andriyash, F. Denef, D. L. Jafferis, and G. W. Moore, Bound state transformation walls, JHEP 1203 (2012) 007, arXiv:1008.3555 [hep-th].

[78] E. Andriyash, F. Denef, D. L. Jafferis, and G. W. Moore, Wallcrossing from supersymmetric galaxies, JHEP 1201 (2012) 115, arXiv:1008.0030 [hep-th].

[79] J. Manschot, B. Pioline, and A. Sen, Wall crossing from Boltzmann black hole halos, JHEP 1107 (2011) 059, arXiv:1011.1258 [hep-th].

[80] J. Manschot, B. Pioline, and A. Sen, From black holes to quivers, JHEP 1211 (2012) 023, arXiv:1207.2230 [hep-th].

[81] G. Lockhart and C. Vafa, Superconformal partition functions and nonperturbative topological strings, arXiv:1210.5909 [hep-th].

[82] T. Dimofte, D. Gaiotto, and S. Gukov, Gauge theories labelled by threemanifolds, arXiv:1108.4389 [hep-th].

[83] S. Cecotti, C. Cordova, and C. Vafa, Braids, walls, and mirrors, arXiv:1110.2115 [hep-th].

[84] B. Keller, The periodicity conjecture for pairs of dynkin diagrams, arXiv: 1001.1531.

[85] D. Xie, 3d $\mathcal{N}=4$ field theory and Hyperkahler manifold, to appear.

School of Natural Sciences, Institute for Advanced Study

Princeton, NJ 08540, USA

E-mail address: dxie@cmsa.fas.harvard.edu 